\title{
Using Biogenic Sulfur Gases as Remotely Detectable Biosignatures on Anoxic Planets
}

\author{
Shawn D. Domagal-Goldman, Victoria S. Meadows, ${ }^{2,3}$ Mark W. Claire, ${ }^{2,3}$ and James F. Kasting ${ }^{3,4}$
}

\begin{abstract}
We used one-dimensional photochemical and radiative transfer models to study the potential of organic sulfur compounds $\left(\mathrm{CS}_{2}\right.$, OCS, $\mathrm{CH}_{3} \mathrm{SH}, \mathrm{CH}_{3} \mathrm{SCH}_{3}$, and $\left.\mathrm{CH}_{3} \mathrm{~S}_{2} \mathrm{CH}_{3}\right)$ to act as remotely detectable biosignatures in anoxic exoplanetary atmospheres. Concentrations of organic sulfur gases were predicted for various biogenic sulfur fluxes into anoxic atmospheres and were found to increase with decreasing UV fluxes. Dimethyl sulfide $\left(\mathrm{CH}_{3} \mathrm{SCH}_{3}\right.$, or DMS) and dimethyl disulfide $\left(\mathrm{CH}_{3} \mathrm{~S}_{2} \mathrm{CH}_{3}\right.$, or DMDS) concentrations could increase to remotely detectable levels, but only in cases of extremely low UV fluxes, which may occur in the habitable zone of an inactive $\mathrm{M}$ dwarf. The most detectable feature of organic sulfur gases is an indirect one that results from an increase in ethane $\left(\mathrm{C}_{2} \mathrm{H}_{6}\right)$ over that which would be predicted based on the planet's methane $\left(\mathrm{CH}_{4}\right)$ concentration. Thus, a characterization mission could detect these organic sulfur gases-and therefore the life that produces them-if it could sufficiently quantify the ethane and methane in the exoplanet's atmosphere. Key Words: Exoplanets-Biosignatures-Anoxic atmospheres-Planetary atmospheres-Remote life detectionPhotochemistry. Astrobiology 11, 419-441.
\end{abstract}

\section{Introduction}

T HE SEARCH for life may soon expand beyond the boundaries of our solar system via the detection of spectral features of "biosignature" gases on extrasolar planets (European Space Agency, 2010; Jet Propulsion Laboratory, 2010; New Worlds Observer Team, 2010). For a gas to be a biosignature it must have a biological production rate that far outpaces abiotic sources and an atmospheric lifetime that allows it to build up to detectable levels. To be detectable, the biosignature gas must have spectral features that are (1) within a wavelength region that can be covered by instrumentation, (2) larger than the signal-to-noise ratio $(\mathrm{S} / \mathrm{N})$ for these instruments, and (3) distinguishable from other spectral features.

For biospheres in which primary productivity is dominated by oxygenic photosynthesis (henceforth referred to as "oxic" biospheres), a number of gases have been identified that meet these criteria: oxygen $\left(\mathrm{O}_{2}\right)$, ozone $\left(\mathrm{O}_{3}\right)$, or both in the presence of reduced species such as methane $\left(\mathrm{CH}_{4}\right)$ (Lovelock, 1965; Des Marais et al., 2002); nitrous oxide $\left(\mathrm{N}_{2} \mathrm{O}\right)$ (Sagan et al., 1993); and methyl chloride $\left(\mathrm{CH}_{3} \mathrm{Cl}\right)$ (Segura et al., 2005). The latter two gases are more difficult to detect in Earth's present atmosphere than are the first two; however, they might be more visible in the atmospheres of oxic Earth-like planets orbiting M stars due to longer atmospheric lifetimes resulting from lower photolysis rates (Segura et al., 2005).

Other biosignatures are needed for detection of "anoxic biospheres" that harbor life but not detectable amounts of atmospheric $\mathrm{O}_{2}$ and $\mathrm{O}_{3}$. Biogenic $\mathrm{CH}_{4}$ could be abundant enough to be detectable in such an atmosphere (Kasting et al., 1983, 2001; Kasting, 2005; Kharecha et al., 2005; Kaltenegger et al., 2007), but its interpretation would be ambiguous because abiotic processes such as serpentinization can also produce $\mathrm{CH}_{4}$ (Berndt et al., 1996; Kasting and Catling, 2003).

From the early history of life on Earth, we know that anoxic biospheres are possible. Studies of early Earth suggest that life was present well before significant $\mathrm{O}_{2}$ accumulated in the atmosphere (Schopf, 1983; Holland, 1984; Farquhar and Wing, 2003; Westall, 2005; Farquhar et al., 2007). This period had vigorous biological activity without significant $\mathrm{O}_{2}$ buildup and may have lasted as long as 1.5 billion years, approximately one-third of Earth's history. This suggests that planets with life, but without $\mathrm{O}_{2} / \mathrm{O}_{3}$, could represent a large fraction of inhabited planets. Thus, the absence of

\footnotetext{
${ }^{1}$ NASA Headquarters, Washington DC.

${ }^{2}$ Astronomy Department, University of Washington, Seattle, Washington.

${ }^{3}$ NASA Astrobiology Institute.

${ }^{4}$ Department of Geosciences, The Pennsylvania State University, University Park, Pennsylvania.
} 
Table 1. List of Reactions in the Photochemical Code, along with the Reaction Rate Constants Used and a Source for the Reaction Rate Constant

\begin{tabular}{|c|c|c|c|}
\hline Rxn. \# & Reaction & Reaction rate constant & Reference \\
\hline 1 & $\mathrm{OCS}+\mathrm{CH} \rightarrow \mathrm{CO}+\mathrm{HCS}$ & $1.99 \cdot 10^{-10} \times \mathrm{e}^{-190 / \mathrm{T}}$ & Zabarnick et al., 1989 \\
\hline 2 & $\mathrm{OCS}+\mathrm{H} \rightarrow \mathrm{CO}+\mathrm{HS}$ & $9.07 \cdot 10^{-12} \times \mathrm{e}^{-1940 / \mathrm{T}}$ & Lee et al., 1977 \\
\hline 3 & $\mathrm{OCS}+\mathrm{O} \rightarrow \mathrm{S}+\mathrm{CO}_{2}$ & $8.3 \cdot 10^{-11} \times \mathrm{e}^{-5530 / \mathrm{T}}$ & Singleton and Cvetanovic, 1988 \\
\hline 4 & $\mathrm{OCS}+\mathrm{O} \rightarrow \mathrm{SO}+\mathrm{CO}$ & $2.1 \cdot 10^{-11} \times \mathrm{e}^{-2200 / \mathrm{T}}$ & Toon et al., 1987 \\
\hline 5 & $\mathrm{OCS}+\mathrm{OH} \rightarrow \mathrm{CO}_{2}+\mathrm{HS}$ & $1.1 \cdot 10^{-13} \times \mathrm{e}^{-1200 / \mathrm{T}}$ & Atkinson et al., 2004 \\
\hline 6 & $\mathrm{OCS}+\mathrm{OH} \rightarrow \mathrm{HS}+\mathrm{CO}_{2}$ & $1.1 \cdot 10^{-13} \times \mathrm{e}^{-1200 / \mathrm{T}}$ & Atkinson et al., 2004 \\
\hline 7 & $\mathrm{OCS}+\mathrm{S} \rightarrow \mathrm{CO}+\mathrm{S}_{2}$ & $1.5 \cdot 10^{-10} \times \mathrm{e}^{-1830 / \mathrm{T}}$ & Schofield, 1973 \\
\hline 8 & $\mathrm{OCS}+\mathrm{S}+\mathrm{M} \rightarrow \mathrm{OCS}_{2}+\mathrm{M}$ & $8.3 \cdot 10^{-33} \times$ den & Basco and Pearson, 1967 \\
\hline 9 & $\mathrm{OCS}_{2}+\mathrm{CO} \rightarrow \mathrm{OCS}+\mathrm{OCS}$ & $3.0 \cdot 10^{-12}$ & Zahnle et al., 2006 \\
\hline 10 & $\mathrm{OCS}_{2}+\mathrm{S} \rightarrow \mathrm{OCS}+\mathrm{S}_{2}$ & $2.0 \cdot 10^{-11}$ & Zahnle et al., 2006 \\
\hline 11 & $\mathrm{C}_{2} \mathrm{H}_{6} \mathrm{~S}+\mathrm{CH}_{3} \rightarrow \mathrm{CH}_{4}+\mathrm{C}_{2} \mathrm{H}_{4}+\mathrm{HS}$ & $6.92 \cdot 10^{-13} \times \mathrm{e}^{-4610 / \mathrm{T}}$ & Arthur and Lee, 1976 \\
\hline 12 & $\mathrm{C}_{2} \mathrm{H}_{6} \mathrm{~S}+\mathrm{H} \rightarrow \mathrm{C}_{2} \mathrm{H}_{5}+\mathrm{H}_{2} \mathrm{~S}$ & $8.49 \cdot 10^{-12} \times \mathrm{e}^{-1200 / \mathrm{T}}$ & Lam et al., 1989 \\
\hline 13 & $\mathrm{C}_{2} \mathrm{H}_{6} \mathrm{~S}+\mathrm{H} \rightarrow \mathrm{CH}_{3} \mathrm{SH}+\mathrm{CH}_{3}$ & $4.81 \cdot 10^{-12} \times \mathrm{e}^{-1100 / \mathrm{T}} \times(\mathrm{T} / 300)^{1.7}$ & Zhang et al., 2005 \\
\hline 14 & $\mathrm{C}_{2} \mathrm{H}_{6} \mathrm{~S}+\mathrm{H} \rightarrow \mathrm{H}_{2}+\mathrm{C}_{2} \mathrm{H}_{4}+\mathrm{HS}$ & $8.34 \cdot 10^{-12} \times \mathrm{e}^{-2212 / \mathrm{T}} \times(\mathrm{T} / 300)^{1.6}$ & Zhang et al., 2005 \\
\hline 15 & $\mathrm{C}_{2} \mathrm{H}_{6} \mathrm{~S}+\mathrm{OH} \rightarrow \mathrm{H}_{2} \mathrm{O}+\mathrm{C}_{2} \mathrm{H}_{4}+\mathrm{HS}$ & $1.13 \cdot 10^{-11} \times \mathrm{e}^{-253 / \mathrm{T}}$ & Atkinson et al., 2004 \\
\hline 16 & $\mathrm{C}_{2} \mathrm{H}_{6} \mathrm{~S}_{2}+\mathrm{H} \rightarrow \mathrm{CH}_{3} \mathrm{SH}+\mathrm{CH}_{3} \mathrm{~S}$ & $9.47 \cdot 10^{-12} \times \mathrm{e}^{-50 / \mathrm{T}}$ & Ekwenchi et al., 1980 \\
\hline 17 & $\mathrm{CH}_{3}+\mathrm{HS} \rightarrow \mathrm{CH}_{3} \mathrm{SH}$ & $1.66 \cdot 10^{-11}$ & Shum and Benson, 1985 \\
\hline 18 & $\mathrm{CH}_{3} \mathrm{~S}+\mathrm{CH}_{3} \mathrm{~S} \rightarrow \mathrm{C}_{2} \mathrm{H}_{6} \mathrm{~S}_{2}$ & $4.00 \cdot 10^{-11}$ & Anastasi et al., 1991 \\
\hline 19 & $\mathrm{CH}_{3} \mathrm{~S}+\mathrm{CO} \rightarrow \mathrm{CH}_{3}+\mathrm{OCS}$ & $2.6 \cdot 10^{-11} \times \mathrm{e}^{-5940 / \mathrm{T}}$ & Assumed same as $k\left(\mathrm{CH}_{3} \mathrm{O}+\mathrm{CO}\right)$ \\
\hline 20 & $\mathrm{CH}_{3} \mathrm{~S}+\mathrm{CS} \rightarrow \mathrm{CH}_{3}+\mathrm{CS}_{2}$ & $2.6 \cdot 10^{-11} \times \mathrm{e}^{-5940 / \mathrm{T}}$ & Assumed same as $k\left(\mathrm{CH}_{3} \mathrm{O}+\mathrm{CO}\right)$ \\
\hline 21 & $\mathrm{CH}_{3} \mathrm{~S}+\mathrm{H}_{2} \mathrm{O}_{2} \rightarrow \mathrm{CH}_{3} \mathrm{SH}+\mathrm{H}_{2} \mathrm{O}$ & $3.01 \cdot 10^{-13}$ & Turnipseed et al., 1996 \\
\hline 22 & $\mathrm{CH}_{3} \mathrm{~S}+\mathrm{HCS} \rightarrow \mathrm{CH}_{3} \mathrm{SH}+\mathrm{CS}$ & $1.18 \cdot 10^{-12} \times \mathrm{e}^{-910 / \mathrm{T}} \times(\mathrm{T} / 300)^{0.65}$ & Liu et al., 2006 \\
\hline 23 & $\mathrm{CH}_{3} \mathrm{~S}+\mathrm{HS} \rightarrow \mathrm{CH}_{3} \mathrm{SH}+\mathrm{S}$ & $1.66 \cdot 10^{-11}$ & Assumed same as $k\left(\mathrm{CH}_{3}+\mathrm{HS}\right)$ \\
\hline 24 & $\mathrm{CH}_{3} \mathrm{SH}+\mathrm{CH}_{3} \rightarrow \mathrm{CH}_{4}+\mathrm{CH}_{3} \mathrm{~S}$ & $2.99 \cdot 10^{-31}$ & Kerr and Trotman-Dickenson, 1957 \\
\hline 25 & $\mathrm{C}_{2} \mathrm{H}_{6} \mathrm{~S}+\mathrm{O} \rightarrow \mathrm{CH}_{3}+\mathrm{CH}_{3}+\mathrm{SO}$ & $1.30 \cdot 10^{-11} \times \mathrm{e}^{-410 / \mathrm{T}} \times(\mathrm{T} / 298)^{1.1}$ & Sander et al., 2006 \\
\hline 26 & $\mathrm{CH}_{3} \mathrm{SH}+\mathrm{O} \rightarrow \mathrm{CH}_{3}+\mathrm{HSO}$ & $1.30 \cdot 10^{-11} \times \mathrm{e}^{-410 / \mathrm{T}} \times(\mathrm{T} / 298)^{1.1}$ & Assumed same as $k\left(\mathrm{C}_{2} \mathrm{H}_{6} \mathrm{~S}+\mathrm{O}\right)$ \\
\hline 27 & $\mathrm{C}_{2} \mathrm{H}_{6} \mathrm{~S}_{2}+\mathrm{O} \rightarrow \mathrm{CH}_{3}+\mathrm{CH}_{3} \mathrm{~S}+\mathrm{SO}$ & $3.90 \cdot 10^{-11} \times \mathrm{e}^{290 / \mathrm{T}} \times(\mathrm{T} / 298)^{1.1}$ & Sander et al., 2006 \\
\hline 28 & $\mathrm{C}_{2} \mathrm{H}_{6} \mathrm{~S}+\mathrm{OH} \rightarrow \mathrm{CH}_{2}{ }^{1}+\mathrm{CH}_{3} \mathrm{~S}+\mathrm{H}_{2} \mathrm{O}$ & $1.10 \cdot 10^{-11} \times \mathrm{e}^{-240 / \mathrm{T}} \times(\mathrm{T} / 298)^{1.1}$ & Sander et al., 2006 \\
\hline 29 & $\mathrm{C}_{2} \mathrm{H}_{6} \mathrm{~S}_{2}+\mathrm{OH} \rightarrow \mathrm{CH}_{3}+\mathrm{CH}_{3} \mathrm{SH}+\mathrm{SO}$ & $6.00 \cdot 10^{-11} \times \mathrm{e}^{400 / \mathrm{T}} \times(\mathrm{T} / 298)^{1.2}$ & Sander et al., 2006 \\
\hline 30 & $\mathrm{CH}_{3} \mathrm{SH}+\mathrm{OH} \rightarrow \mathrm{CH}_{3} \mathrm{~S}+\mathrm{H}_{2} \mathrm{O}$ & $9.90 \cdot 10^{-12} \times \mathrm{e}^{360 / \mathrm{T}} \times(\mathrm{T} / 298)^{1.07}$ & Sander et al., 2006 \\
\hline 31 & $\mathrm{C}_{2} \mathrm{H}_{6} \mathrm{~S}+\mathrm{O} \rightarrow \mathrm{CH}_{3}+\mathrm{CH}_{3}+\mathrm{SO}$ & $1.30 \cdot 10^{-11} \times \mathrm{e}^{-410 / \mathrm{T}} \times(\mathrm{T} / 298)^{1.1}$ & Sander et al., 2006 \\
\hline 32 & $\mathrm{CH}_{3} \mathrm{SH}+\mathrm{O} \rightarrow \mathrm{CH}_{3}+\mathrm{HSO}$ & $1.30 \cdot 10^{-11} \times \mathrm{e}^{-410 / \mathrm{T}} \times(\mathrm{T} / 298)^{1.1}$ & Assumed same as $k\left(\mathrm{C}_{2} \mathrm{H}_{6} \mathrm{~S}+\mathrm{O}\right)$ \\
\hline 33 & $\mathrm{CH}_{3} \mathrm{SH}+\mathrm{H} \rightarrow \mathrm{CH}_{3}+\mathrm{H}_{2} \mathrm{~S}$ & $1.5 \cdot 10^{-11} \times \mathrm{e}^{-840 / \mathrm{T}}$ & Amano et al., 1983 \\
\hline 34 & $\mathrm{CH}_{3} \mathrm{SH}+\mathrm{H} \rightarrow \mathrm{H}_{2}+\mathrm{CH}_{3} \mathrm{~S}$ & $4.82 \cdot 10^{-11} \times \mathrm{e}^{-1310 / \mathrm{T}}$ & Amano et al., 1983 \\
\hline 35 & $\mathrm{CH}_{3} \mathrm{SH}+\mathrm{OH} \rightarrow \mathrm{H}_{2} \mathrm{O}+\mathrm{CH}_{3} \mathrm{~S}$ & $9.9 \cdot 10^{-12} \times \mathrm{e}^{360 / \mathrm{T}}$ & DeMore and Yung, 1982 \\
\hline 36 & $\mathrm{CH}+\mathrm{CS}_{2} \rightarrow \mathrm{HCS}+\mathrm{CS}$ & $3.49 \cdot 10^{-10} \times \mathrm{e}^{-40 / \mathrm{T}}$ & Zabarnick et al., 1989 \\
\hline 37 & $\mathrm{CS}+\mathrm{HS} \rightarrow \mathrm{CS}_{2}+\mathrm{H}$ & $1.5 \cdot 10^{-13} \times(1+0.6 \times$ den $)$ & Assumed same as $k(\mathrm{CO}+\mathrm{OH})$ \\
\hline 38 & $\mathrm{CS}+\mathrm{O} \rightarrow \mathrm{CO}+\mathrm{S}$ & $2.7 \cdot 10^{-10} \times \mathrm{e}^{-760 / \mathrm{T}}$ & Atkinson et al., 2004 \\
\hline 39 & $\mathrm{CS}+\mathrm{O}_{2} \rightarrow \mathrm{CO}+\mathrm{SO}$ & $5 \cdot 10^{-20}$ & Wine et al., 1981 \\
\hline 40 & $\mathrm{CS}+\mathrm{O}_{2} \rightarrow \mathrm{OCS}+\mathrm{O}$ & $4 \cdot 10^{-19}$ & Wine et al., 1981 \\
\hline 41 & $\mathrm{CS}+\mathrm{O}_{3} \rightarrow \mathrm{CO}+\mathrm{SO}_{2}$ & $3 \cdot 10^{-12}$ & Wine et al., 1981 \\
\hline 42 & $\mathrm{CS}+\mathrm{O}_{3} \rightarrow \mathrm{OCS}+\mathrm{O}_{2}$ & $3 \cdot 10^{-12}$ & Wine et al., 1981 \\
\hline 43 & $\mathrm{CS}+\mathrm{O}_{3} \rightarrow \mathrm{SO}+\mathrm{CO}_{2}$ & $3 \cdot 10^{-12}$ & Wine et al., 1981 \\
\hline 44 & $\mathrm{CS}_{2}+\mathrm{O} \rightarrow \mathrm{CO}+\mathrm{S}_{2}$ & $5.81 \cdot 10^{-14}$ & Singleton and Cvetanovic, 1988 \\
\hline 45 & $\mathrm{CS}_{2}+\mathrm{O} \rightarrow \mathrm{OCS}+\mathrm{S}$ & $3 \cdot 10^{-12} \times \mathrm{e}^{-650 / \mathrm{T}}$ & Toon et al., 1987 \\
\hline 46 & $\mathrm{CS}_{2}+\mathrm{O} \rightarrow \mathrm{SO}+\mathrm{CS}$ & $3.2 \cdot 10^{-11} \times \mathrm{e}^{-650 / \mathrm{T}}$ & Toon et al., 1987 \\
\hline 47 & $\mathrm{CS}_{2}+\mathrm{OH} \rightarrow \mathrm{OCS}+\mathrm{HS}$ & $2 \cdot 10^{-15}$ & Atkinson et al., 2004 \\
\hline 48 & $\mathrm{CS}_{2}+\mathrm{S} \rightarrow \mathrm{CS}+\mathrm{S}_{2}$ & $1.9 \cdot 10^{-14} \times \mathrm{e}^{-580 / \mathrm{T}} \times(\mathrm{T} / 300)^{3.97}$ & Woiki and Roth, 1995 \\
\hline 49 & $\mathrm{CS}_{2}+\mathrm{SO} \rightarrow \mathrm{OCS}+\mathrm{S}_{2}$ & $2.4 \cdot 10^{-13} \times \mathrm{e}^{-2370 / \mathrm{T}}$ & Assumed same as $k\left(\mathrm{SO}^{*}+\mathrm{O}_{2}\right)$ \\
\hline 50 & $\mathrm{CS}_{2}{ }^{*}+\mathrm{CS}_{2} \rightarrow \mathrm{CS}+\mathrm{CS}+\mathrm{S}_{2}$ & $1 \cdot 10^{-12}$ & Assumed same as $k\left(\mathrm{CS}_{2}^{*}+\mathrm{CS}_{2}\right.$ \\
\hline 51 & $\mathrm{CS}_{2}{ }^{*}+\mathrm{M} \rightarrow \mathrm{CS}_{2}+\mathrm{M}$ & $2.5 \cdot 10^{-11}$ & Wine et al., 1981 \\
\hline 52 & $\mathrm{CS}_{2}^{*}+\mathrm{O}_{2} \rightarrow \mathrm{CS}+\mathrm{SO}_{2}$ & $1 \cdot 10^{-12}$ & Wine et al., 1981 \\
\hline 53 & $\mathrm{C}+\mathrm{HS} \rightarrow \mathrm{CS}+\mathrm{H}$ & $4 \cdot 10^{-11}$ & Assumed same as $k(\mathrm{C}+\mathrm{OH})$ \\
\hline 54 & $\mathrm{C}+\mathrm{S}_{2} \rightarrow \mathrm{CS}+\mathrm{S}$ & $3.3 \cdot 10^{-11}$ & Assumed same as $k\left(\mathrm{C}+\mathrm{O}_{2}\right)$ \\
\hline 55 & $\mathrm{C}_{2}+\mathrm{S} \rightarrow \mathrm{C}+\mathrm{CS}$ & $5 \cdot 10^{-11}$ & Assumed same as $k\left(\mathrm{C}_{2}+\mathrm{O}\right)$ \\
\hline 56 & $\mathrm{C}_{2}+\mathrm{S}_{2} \rightarrow \mathrm{CS}+\mathrm{CS}$ & $1.5 \cdot 10^{-11} \times \mathrm{e}^{-550 / \mathrm{T}}$ & Assumed same as $k\left(\mathrm{C}_{2}+\mathrm{O}_{2}\right)$ \\
\hline 57 & $\mathrm{CH}+\mathrm{S} \rightarrow \mathrm{CS}+\mathrm{H}$ & $9.5 \cdot 10^{-11}$ & Assumed same as $k\left(\mathrm{CH}+\mathrm{CS}_{2}\right)$ \\
\hline 58 & $\mathrm{CH}+\mathrm{S}_{2} \rightarrow \mathrm{CS}+\mathrm{HS}$ & $5.9 \cdot 10^{-11}$ & Assumed same as $k\left(\mathrm{CH}+\mathrm{O}_{2}\right)$ \\
\hline 59 & $\mathrm{CH}_{2}^{1}+\mathrm{S}_{2} \rightarrow \mathrm{HCS}+\mathrm{HS}$ & $3 \cdot 10^{-11}$ & Assumed same as $k\left(\mathrm{CH}_{2}^{1}+\mathrm{O}_{2}\right)$ \\
\hline 60 & $\mathrm{CH}_{3}+\mathrm{HCS} \rightarrow \mathrm{CH}_{4}+\mathrm{CS}$ & $8.2 \cdot 10^{-11}$ & Assumed same as $k\left(\mathrm{CH}_{3}+\mathrm{HCO}\right)$ \\
\hline 61 & $\mathrm{H}+\mathrm{CS}+\mathrm{M} \rightarrow \mathrm{HCS}+\mathrm{M}$ & $2.0 \cdot 10^{-33} \times \mathrm{e}^{-850 / \mathrm{T}} \times$ den & Assumed same as $k(\mathrm{H}+\mathrm{CO})$ \\
\hline
\end{tabular}


Table 1. (Continued)

\begin{tabular}{|c|c|c|c|}
\hline Rxn. \# & Reaction & Reaction rate constant & Reference \\
\hline 62 & $\mathrm{H}+\mathrm{HCS} \rightarrow \mathrm{H}_{2}+\mathrm{CS}$ & $1.2 \cdot 10^{-10}$ & Assumed same as $k(\mathrm{H}+\mathrm{HCO})$ \\
\hline 63 & $\mathrm{HS}+\mathrm{CO} \rightarrow \mathrm{OCS}+\mathrm{H}$ & $4.2 \cdot 10^{-14} \times \mathrm{e}^{-7650 / \mathrm{T}}$ & Kurbanov and Mamedov, 1995 \\
\hline 64 & $\mathrm{HS}+\mathrm{HCS} \rightarrow \mathrm{H}_{2} \mathrm{~S}+\mathrm{CS}$ & $5.0 \cdot 10^{-11}$ & Assumed same as $k(\mathrm{HS}+\mathrm{HCO})$ \\
\hline 65 & $\mathrm{OCS}+\mathrm{CH} \rightarrow \mathrm{CO}+\mathrm{HCS}$ & $1.99 \cdot 10^{-10} \times \mathrm{e}^{-190 / \mathrm{T}}$ & Zabarnick et al., 1989 \\
\hline 66 & $\mathrm{~S}+\mathrm{CO}+\mathrm{M} \rightarrow \mathrm{OCS}+\mathrm{M}$ & $6.5 \cdot 10^{-33} \times \mathrm{e}^{-2180 / \mathrm{T}} \times$ den & Assumed same as $k(\mathrm{CO}+\mathrm{O})$ \\
\hline 67 & $\mathrm{~S}+\mathrm{HCS} \rightarrow \mathrm{H}+\mathrm{CS}_{2}$ & $1.0 \cdot 10^{-10}$ & $\begin{array}{l}\text { Assumed same as } k(\mathrm{O}+ \\
\left.\quad \mathrm{HCO} \rightarrow \mathrm{H}+\mathrm{CO}_{2}\right)\end{array}$ \\
\hline 68 & $\mathrm{~S}+\mathrm{HCS} \rightarrow \mathrm{HS}+\mathrm{CS}$ & $5.0 \cdot 10^{-11}$ & $\begin{array}{l}\text { Assumed same as } k(\mathrm{O}+ \\
\mathrm{HCO} \rightarrow \mathrm{HS}+\mathrm{CO})\end{array}$ \\
\hline 69 & $2 \mathrm{CH}_{2}^{3} \rightarrow \mathrm{C}_{2} \mathrm{H}_{2}+\mathrm{H}_{2}$ & $5.3 \cdot 10^{-11}$ & Braun et al., 1970 \\
\hline 70 & $\mathrm{C}+\mathrm{H}_{2}+\mathrm{M} \rightarrow \mathrm{CH}_{2}^{3}+\mathrm{M}$ & $\begin{array}{l}k_{0}=8.75 \cdot 10^{-31} \times \mathrm{e}^{524 / \mathrm{T}} \\
k_{\infty}=8.3 \cdot 10^{-1}\end{array}$ & Zahnle, 1986 \\
\hline 71 & $\mathrm{C}+\mathrm{O}_{2} \rightarrow \mathrm{CO}+\mathrm{O}$ & $3.3 \cdot 10^{-11}$ & Donovan and Husain, 1970 \\
\hline 72 & $\mathrm{C}+\mathrm{OH} \rightarrow \mathrm{CO}+\mathrm{H}$ & $4 \cdot 10^{-11}$ & Giguere and Huebner, 1978 \\
\hline 73 & $\mathrm{C}_{2}+\mathrm{CH}_{4} \rightarrow \mathrm{C}_{2} \mathrm{H}+\mathrm{CH}_{3}$ & $5.05 \cdot 10^{-11} \times \mathrm{e}^{-297 / \mathrm{T}}$ & Pitts et al., 1982 \\
\hline 74 & $\mathrm{C}_{2}+\mathrm{H}_{2} \rightarrow \mathrm{C}_{2} \mathrm{H}+\mathrm{H}$ & $1.77 \cdot 10^{-10} \times \mathrm{e}^{-1469 / \mathrm{T}}$ & Pitts et al., 1982 \\
\hline 75 & $\mathrm{C}_{2}+\mathrm{O} \rightarrow \mathrm{C}+\mathrm{CO}$ & $5 \cdot 10^{-11}$ & Prasad and Huntress, 1980 \\
\hline 76 & $\mathrm{C}_{2}+\mathrm{O}_{2} \rightarrow \mathrm{CO}+\mathrm{CO}$ & $1.5 \cdot 10^{-11} \times \mathrm{e}^{-550 / \mathrm{T}}$ & Baughcum and Oldenborg, 1984 \\
\hline 77 & $\mathrm{C}_{2} \mathrm{H}+\mathrm{C}_{2} \mathrm{H}_{2} \rightarrow \mathrm{HCAER}+\mathrm{H}$ & $1.5 \cdot 10^{-10}$ & Stephens et al., 1987 \\
\hline 78 & $\mathrm{C}_{2} \mathrm{H}+\mathrm{C}_{2} \mathrm{H}_{6} \rightarrow \mathrm{C}_{2} \mathrm{H}_{2}+\mathrm{C}_{2} \mathrm{H}_{5}$ & $3.6 \cdot 10^{-11}$ & Lander et al., 1990 \\
\hline 79 & $\mathrm{C}_{2} \mathrm{H}+\mathrm{C}_{3} \mathrm{H}_{8} \rightarrow \mathrm{C}_{2} \mathrm{H}_{2}+\mathrm{C}_{3} \mathrm{H}_{7}$ & $1.4 \cdot 10^{-11}$ & Okabe, 1983 \\
\hline 80 & $\mathrm{C}_{2} \mathrm{H}+\mathrm{CH}_{2} \mathrm{CCH}_{2} \rightarrow \mathrm{HCAER}+\mathrm{H}$ & $1.5 \cdot 10^{-10}$ & Pavlov et al., 2001 \\
\hline 81 & $\mathrm{C}_{2} \mathrm{H}+\mathrm{CH}_{4} \rightarrow \mathrm{C}_{2} \mathrm{H}_{2}+\mathrm{CH}_{3}$ & $6.94 \cdot 10^{-12} \times \mathrm{e}^{-250 / \mathrm{T}}$ & Allen et al., 1992; Lander et al., 1990 \\
\hline 82 & $\mathrm{C}_{2} \mathrm{H}+\mathrm{H}+\mathrm{M} \rightarrow \mathrm{C}_{2} \mathrm{H}_{2}+\mathrm{M}$ & $\begin{aligned} k_{0}= & 2.64 \cdot 10^{-26} \times \mathrm{e}^{-721 / \mathrm{T}} \\
& \times(\mathrm{T} / 300)^{-3.1} \\
k_{\infty} & =3.0 \cdot 10^{-10}\end{aligned}$ & Tsang and Hampson, 1986 \\
\hline 83 & $\mathrm{C}_{2} \mathrm{H}+\mathrm{H}_{2} \rightarrow \mathrm{C}_{2} \mathrm{H}_{2}+\mathrm{H}$ & $5.58 \cdot 10^{-11} \times \mathrm{e}^{-1443 / \mathrm{T}}$ & Allen et al., 1992; Stephens et al., 1987 \\
\hline 84 & $\mathrm{C}_{2} \mathrm{H}+\mathrm{O} \rightarrow \mathrm{CO}+\mathrm{CH}$ & $1 \cdot 10^{-10} \times \mathrm{e}^{-250 / \mathrm{T}}$ & Zahnle, 1986 \\
\hline 85 & $\mathrm{C}_{2} \mathrm{H}+\mathrm{O}_{2} \rightarrow \mathrm{CO}+\mathrm{HCO}$ & $2 \cdot 10^{-11}$ & Brown and Laufer, 1981 \\
\hline 86 & $\mathrm{C}_{2} \mathrm{H}_{2}+\mathrm{H}+\mathrm{M} \rightarrow \mathrm{C}_{2} \mathrm{H}_{3}+\mathrm{M}$ & $\begin{array}{l}k_{0}=2.6 \cdot 10^{-31} \\
k_{\infty}=8.3 \cdot 10^{-11} \times \mathrm{e}^{-1374 / \mathrm{T}}\end{array}$ & Romani et al., 1993 \\
\hline 87 & $\mathrm{C}_{2} \mathrm{H}_{2}+\mathrm{O} \rightarrow \mathrm{CH}_{2}^{3}+\mathrm{CO}$ & $2.9 \cdot 10^{-11} \times \mathrm{e}^{-1600 / \mathrm{T}}$ & Zahnle, 1986 \\
\hline 88 & $\mathrm{C}_{2} \mathrm{H}_{2}+\mathrm{OH}+\mathrm{M} \rightarrow \mathrm{C}_{2} \mathrm{H}_{2} \mathrm{OH}+\mathrm{M}$ & $\begin{array}{l}k_{0}=5.5 \cdot 10^{-30} \\
k_{\infty}=8.3 \cdot 10^{-13} \times(\mathrm{T} / 300)^{-2}\end{array}$ & Sander et al., 2006 \\
\hline 89 & $\mathrm{C}_{2} \mathrm{H}_{2}+\mathrm{OH}+\mathrm{M} \rightarrow \mathrm{CH}_{2} \mathrm{CO}+\mathrm{H}+\mathrm{M}$ & $\begin{array}{l}k_{0}=5.8 \cdot 10^{-31} \times \mathrm{e}^{1258 / \mathrm{T}} \\
k_{\infty}=1.4 \cdot 10^{-12} \times \mathrm{e}^{388 / \mathrm{T}}\end{array}$ & Perry and Williamson, 1982 \\
\hline 90 & $\mathrm{C}_{2} \mathrm{H}_{2}+\mathrm{OH} \rightarrow \mathrm{CO}+\mathrm{CH}_{3}$ & $2 \cdot 10^{-12} \times \mathrm{e}^{-250 / \mathrm{T}}$ & Hampson and Garvin, 1977 \\
\hline 91 & $\mathrm{C}_{2} \mathrm{H}_{2} \mathrm{OH}+\mathrm{H} \rightarrow \mathrm{H}_{2}+\mathrm{CH}_{2} \mathrm{CO}$ & $3.3 \cdot 10^{-11} \times \mathrm{e}^{-2000 / \mathrm{T}}$ & Miller et al., 1982 \\
\hline 92 & $\mathrm{C}_{2} \mathrm{H}_{2} \mathrm{OH}+\mathrm{H} \rightarrow \mathrm{H}_{2} \mathrm{O}+\mathrm{C}_{2} \mathrm{H}_{2}$ & $5 \cdot 10^{-11}$ & Miller et al., 1982 \\
\hline 93 & $\mathrm{C}_{2} \mathrm{H}_{2} \mathrm{OH}+\mathrm{O} \rightarrow \mathrm{OH}+\mathrm{CH}_{2} \mathrm{CO}$ & $3.3 \cdot 10^{-11} \times \mathrm{e}^{-2000 / \mathrm{T}}$ & Miller et al., 1982 \\
\hline 94 & $\mathrm{C}_{2} \mathrm{H}_{2} \mathrm{OH}+\mathrm{OH} \rightarrow \mathrm{H}_{2} \mathrm{O}+\mathrm{CH}_{2} \mathrm{CO}$ & $1.7 \cdot 10^{-11} \times \mathrm{e}^{-1000 / \mathrm{T}}$ & Miller et al., 1982 \\
\hline 95 & $\mathrm{C}_{2} \mathrm{H}_{3}+\mathrm{C}_{2} \mathrm{H}_{3} \rightarrow \mathrm{C}_{2} \mathrm{H}_{4}+\mathrm{C}_{2} \mathrm{H}_{2}$ & $2.4 \cdot 10^{-11}$ & Fahr et al., 1991 \\
\hline 96 & $\mathrm{C}_{2} \mathrm{H}_{3}+\mathrm{C}_{2} \mathrm{H}_{5} \rightarrow \mathrm{C}_{2} \mathrm{H}_{4}+\mathrm{C}_{2} \mathrm{H}_{4}$ & $3 \cdot 10^{-12}$ & Laufer et al., 1983 \\
\hline 97 & $\mathrm{C}_{2} \mathrm{H}_{3}+\mathrm{C}_{2} \mathrm{H}_{5}+\mathrm{M} \rightarrow \mathrm{CH}_{3}+\mathrm{C}_{3} \mathrm{H}_{5}+\mathrm{M}$ & $\begin{array}{l}k_{0}=1.9 \cdot 10^{-27} \\
k_{\infty}=2.5 \cdot 10^{-11}\end{array}$ & Romani et al., 1993 \\
\hline 98 & $\mathrm{C}_{2} \mathrm{H}_{3}+\mathrm{C}_{2} \mathrm{H}_{6} \rightarrow \mathrm{C}_{2} \mathrm{H}_{4}+\mathrm{C}_{2} \mathrm{H}_{5}$ & $3 \cdot 10^{-13} \times \mathrm{e}^{-5170 / \mathrm{T}}$ & Kasting et al., 1983 \\
\hline 99 & $\mathrm{C}_{2} \mathrm{H}_{3}+\mathrm{CH}_{3} \rightarrow \mathrm{C}_{2} \mathrm{H}_{2}+\mathrm{CH}_{4}$ & $34 \cdot 10^{-11}$ & Fahr et al., 1991 \\
\hline 100 & $\mathrm{C}_{2} \mathrm{H}_{3}+\mathrm{CH}_{3}+\mathrm{M} \rightarrow \mathrm{C}_{3} \mathrm{H}_{6}+\mathrm{M}$ & $\begin{array}{l}k_{0}=1.3 \cdot 10^{-22} \\
k_{\infty}=1.2 \cdot 10^{-10}\end{array}$ & Raymond et al., 2006 \\
\hline 101 & $\mathrm{C}_{2} \mathrm{H}_{3}+\mathrm{CH}_{4} \rightarrow \mathrm{C}_{2} \mathrm{H}_{4}+\mathrm{CH}_{3}$ & $2.4 \cdot 10^{-24} \times \mathrm{e}^{-2754 / \mathrm{T}} \times \mathrm{T}^{4.02}$ & Tsang and Hampson, 1986 \\
\hline 102 & $\mathrm{C}_{2} \mathrm{H}_{3}+\mathrm{H} \rightarrow \mathrm{C}_{2} \mathrm{H}_{2}+\mathrm{H}_{2}$ & $3.3 \cdot 10^{-11}$ & Warnatz, 1984 \\
\hline 103 & $\mathrm{C}_{2} \mathrm{H}_{3}+\mathrm{H}_{2} \rightarrow \mathrm{C}_{2} \mathrm{H}_{4}+\mathrm{H}$ & $2.6 \cdot 10^{-13} \times \mathrm{e}^{-2646 / \mathrm{T}}$ & Allen et al., 1992 \\
\hline 104 & $\mathrm{C}_{2} \mathrm{H}_{3}+\mathrm{O} \rightarrow \mathrm{CH}_{2} \mathrm{CO}+\mathrm{H}$ & $5.5 \cdot 10^{-11}$ & Hoyermann et al., 1981 \\
\hline 105 & $\mathrm{C}_{2} \mathrm{H}_{3}+\mathrm{OH} \rightarrow \mathrm{C}_{2} \mathrm{H}_{2}+\mathrm{H}_{2} \mathrm{O}$ & $8.3 \cdot 10^{-12}$ & Benson and Haugen, 1967 \\
\hline 106 & $\mathrm{C}_{2} \mathrm{H}_{4}+\mathrm{H}+\mathrm{M} \rightarrow \mathrm{C}_{2} \mathrm{H}_{5}+\mathrm{M}$ & $\begin{array}{l}k_{0}=2.15 \cdot 10^{-29} \times \mathrm{e}^{-349 / \mathrm{T}} \\
k_{\infty}=4.95 \cdot 10^{-11} \times \mathrm{e}^{-1051 / \mathrm{T}}\end{array}$ & Lightfoot and Pilling, 1987 \\
\hline 107 & $\mathrm{C}_{2} \mathrm{H}_{4}+\mathrm{O} \rightarrow \mathrm{HCO}+\mathrm{CH}_{3}$ & $5.5 \cdot 10^{-12} \times \mathrm{e}^{-565 / \mathrm{T}}$ & Hampson and Garvin, 1977 \\
\hline 108 & $\mathrm{C}_{2} \mathrm{H}_{4}+\mathrm{OH}+\mathrm{M} \rightarrow \mathrm{C}_{2} \mathrm{H}_{4} \mathrm{OH}+\mathrm{M}$ & $\begin{array}{l}k_{0}=1.0 \cdot 10^{-28} \times(\mathrm{T} / 300)^{4.5} \\
k_{\infty}=8.8 \cdot 10^{-12} \times(\mathrm{T} / 300)^{0.85}\end{array}$ & Sander et al., 2006 \\
\hline 109 & $\mathrm{C}_{2} \mathrm{H}_{4}+\mathrm{OH} \rightarrow \mathrm{H}_{2} \mathrm{CO}+\mathrm{CH}_{3}$ & $2.2 \cdot 10^{-12} \times \mathrm{e}^{385 / \mathrm{T}}$ & Hampson and Garvin, 1977 \\
\hline 110 & $\mathrm{C}_{2} \mathrm{H}_{4} \mathrm{OH}+\mathrm{H} \rightarrow \mathrm{H}_{2}+\mathrm{CH}_{3} \mathrm{CHO}$ & $3.3 \cdot 10^{-11} \times \mathrm{e}^{-2000 / \mathrm{T}}$ & Zahnle and Kasting, 1986 \\
\hline 111 & $\mathrm{C}_{2} \mathrm{H}_{4} \mathrm{OH}+\mathrm{H} \rightarrow \mathrm{H}_{2} \mathrm{O}+\mathrm{C}_{2} \mathrm{H}_{4}$ & $5 \cdot 10^{-11}$ & Miller et al., 1982 \\
\hline
\end{tabular}


Table 1. (Continued)

\begin{tabular}{|c|c|c|c|}
\hline Rxn. \# & Reaction & Reaction rate constant & Reference \\
\hline 112 & $\mathrm{C}_{2} \mathrm{H}_{4} \mathrm{OH}+\mathrm{O} \rightarrow \mathrm{OH}+\mathrm{CH}_{3} \mathrm{CHO}$ & $3.3 \cdot 10^{-11} \times \mathrm{e}^{-2000 / \mathrm{T}}$ & Zahnle and Kasting, 1986 \\
\hline 113 & $\mathrm{C}_{2} \mathrm{H}_{4} \mathrm{OH}+\mathrm{OH} \rightarrow \mathrm{H}_{2} \mathrm{O}+\mathrm{CH}_{3} \mathrm{CHO}$ & $1.7 \cdot 10^{-11} \times \mathrm{e}^{-1000 / \mathrm{T}}$ & Zahnle and Kasting, 1986 \\
\hline 114 & $\mathrm{C}_{2} \mathrm{H}_{5}+\mathrm{C}_{2} \mathrm{H}_{3} \rightarrow \mathrm{C}_{2} \mathrm{H}_{6}+\mathrm{C}_{2} \mathrm{H}_{2}$ & $6 \cdot 10^{-12}$ & Laufer et al., 1983 \\
\hline 115 & $\mathrm{C}_{2} \mathrm{H}_{5}+\mathrm{C}_{2} \mathrm{H}_{5} \rightarrow \mathrm{C}_{2} \mathrm{H}_{6}+\mathrm{C}_{2} \mathrm{H}_{4}$ & $2.3 \cdot 10^{-12}$ & Tsang and Hampson, 1986 \\
\hline 116 & $\mathrm{C}_{2} \mathrm{H}_{5}+\mathrm{CH}_{3} \rightarrow \mathrm{C}_{2} \mathrm{H}_{4}+\mathrm{CH}_{4}$ & $1.88 \cdot 10^{-12} \times(\mathrm{T} / 300)^{-0.5}$ & Romani et al., 1993 \\
\hline 117 & $\mathrm{C}_{2} \mathrm{H}_{5}+\mathrm{CH}_{3}+\mathrm{M} \rightarrow \mathrm{C}_{3} \mathrm{H}_{8}+\mathrm{M}$ & $\begin{array}{l}k_{0}=3.9 \cdot 10^{-10} \times(\mathrm{T} / 300)^{2.5} \\
k_{\infty}=1.4 \cdot 10^{-8} \times(\mathrm{T} / 300)^{0.5}\end{array}$ & Romani et al., 1993 \\
\hline 118 & $\mathrm{C}_{2} \mathrm{H}_{5}+\mathrm{H} \rightarrow \mathrm{C}_{2} \mathrm{H}_{4}+\mathrm{H}_{2}$ & $3 \cdot 10^{-12}$ & Tsang and Hampson, 1986 \\
\hline 119 & $\mathrm{C}_{2} \mathrm{H}_{5}+\mathrm{H}+\mathrm{M} \rightarrow \mathrm{C}_{2} \mathrm{H}_{6}+\mathrm{M}$ & $\begin{array}{l}k_{0}=5.5 \cdot 10^{-23} \times \mathrm{e}^{-1040 / \mathrm{T}} \\
k_{\infty}=1.5 \cdot 10^{-10}\end{array}$ & Gladstone et al., 1996 \\
\hline 120 & $\mathrm{C}_{2} \mathrm{H}_{5}+\mathrm{H} \rightarrow \mathrm{CH}_{3}+\mathrm{CH}_{3}$ & $7.95 \cdot 10^{-11}$ & Gladstone et al., 1996 \\
\hline 121 & $\mathrm{C}_{2} \mathrm{H}_{5}+\mathrm{HCO} \rightarrow \mathrm{C}_{2} \mathrm{H}_{6}+\mathrm{CO}$ & $5 \cdot 10^{-11}$ & Pavlov et al., 2001 \\
\hline 122 & $\mathrm{C}_{2} \mathrm{H}_{5}+\mathrm{HNO} \rightarrow \mathrm{C}_{2} \mathrm{H}_{6}+\mathrm{NO}$ & $3 \cdot 10^{-14}$ & Pavlov et al., 2001 \\
\hline 123 & $\mathrm{C}_{2} \mathrm{H}_{5}+\mathrm{O} \rightarrow \mathrm{CH}_{3}+\mathrm{HCO}+\mathrm{H}$ & $1.1 \cdot 10^{-10}$ & Pavlov et al., 2001 \\
\hline 124 & $\mathrm{C}_{2} \mathrm{H}_{5}+\mathrm{O} \rightarrow \mathrm{CH}_{3} \mathrm{CHO}+\mathrm{H}$ & $1.33 \cdot 10^{-10}$ & Tsang and Hampson, 1986 \\
\hline \multirow[t]{2}{*}{125} & $\begin{array}{l}\mathrm{C}_{2} \mathrm{H}_{5}+\mathrm{O}_{2}+\mathrm{M} \rightarrow \mathrm{CH}_{3}+ \\
\mathrm{HCO}+\mathrm{OH}+\mathrm{M}\end{array}$ & $k_{0}=1.5 \cdot 10^{-28} \times(\mathrm{T} / 300)^{3.0}$ & Sander et al., 2006 \\
\hline & & $k_{\infty}=8 \cdot 10^{-12}$ & \\
\hline 126 & $\mathrm{C}_{2} \mathrm{H}_{5}+\mathrm{OH} \rightarrow \mathrm{CH}_{3} \mathrm{CHO}+\mathrm{H}_{2}$ & $1 \cdot 10^{-10}$ & Pavlov et al., 2001 \\
\hline 127 & $\mathrm{C}_{2} \mathrm{H}_{5}+\mathrm{OH} \rightarrow \mathrm{C}_{2} \mathrm{H}_{4}+\mathrm{H}_{2} \mathrm{O}$ & $4.0 \cdot 10^{-11}$ & Pavlov et al., 2001 \\
\hline 128 & $\mathrm{C}_{2} \mathrm{H}_{6}+\mathrm{O} \rightarrow \mathrm{C}_{2} \mathrm{H}_{5}+\mathrm{OH}$ & $8.62 \cdot 10^{-12} \times \mathrm{e}^{-2920 / \mathrm{T}} \times(\mathrm{T} / 300)^{1.5}$ & Baulch et al., 1994 \\
\hline 129 & $\mathrm{C}_{2} \mathrm{H}_{6}+\mathrm{O}^{1} \mathrm{D} \rightarrow \mathrm{C}_{2} \mathrm{H}_{5}+\mathrm{OH}$ & $6.29 \cdot 10^{-10}$ & Matsumi et al., 1993 \\
\hline 130 & $\mathrm{C}_{2} \mathrm{H}_{6}+\mathrm{OH} \rightarrow \mathrm{C}_{2} \mathrm{H}_{5}+\mathrm{H}_{2} \mathrm{O}$ & $8.54 \cdot 10^{-12} \times \mathrm{e}^{-1070 / \mathrm{T}}$ & Sander et al., 2006 \\
\hline 131 & $\mathrm{C}_{3} \mathrm{H}_{2}+\mathrm{H}+\mathrm{M} \rightarrow \mathrm{C}_{3} \mathrm{H}_{3}+\mathrm{M}$ & $\begin{array}{l}k_{0}=1.7 \cdot 10^{-26} \\
k_{\infty}=1.5 \cdot 10^{-10}\end{array}$ & Yung et al., 1984 \\
\hline 132 & $\mathrm{C}_{3} \mathrm{H}_{3}+\mathrm{H}+\mathrm{M} \rightarrow \mathrm{CH}_{2} \mathrm{CCH}_{2}+\mathrm{M}$ & $\begin{array}{l}k_{0}=1.7 \cdot 10^{-26} \\
k_{\infty}=1.5 \cdot 10^{-10}\end{array}$ & Yung et al., 1984 \\
\hline 133 & $\mathrm{C}_{3} \mathrm{H}_{3}+\mathrm{H}+\mathrm{M} \rightarrow \mathrm{CH}_{3} \mathrm{C}_{2} \mathrm{H}+\mathrm{M}$ & $\begin{array}{l}k_{0}=1.7 \cdot 10^{-26} \\
k_{\infty}=1.5 \cdot 10^{-10}\end{array}$ & Yung et al., 1984 \\
\hline 134 & $\mathrm{C}_{3} \mathrm{H}_{5}+\mathrm{CH}_{3} \rightarrow \mathrm{CH}_{2} \mathrm{CCH}_{2}+\mathrm{CH}_{4}$ & $4.5 \cdot 10^{-12}$ & Yung et al., 1984 \\
\hline 135 & $\mathrm{C}_{3} \mathrm{H}_{5}+\mathrm{CH}_{3} \rightarrow \mathrm{CH}_{3} \mathrm{C}_{2} \mathrm{H}+\mathrm{CH}_{4}$ & $4.5 \cdot 10^{-12}$ & Yung et al., 1984 \\
\hline 136 & $\mathrm{C}_{3} \mathrm{H}_{5}+\mathrm{H}+\mathrm{M} \rightarrow \mathrm{C}_{3} \mathrm{H}_{6}+\mathrm{M}$ & $\begin{array}{l}k_{0}=1.0 \cdot 10^{-28} \\
k_{\infty}=1.0 \cdot 10^{-11}\end{array}$ & Yung et al., 1984 \\
\hline 137 & $\mathrm{C}_{3} \mathrm{H}_{5}+\mathrm{H} \rightarrow \mathrm{CH}_{2} \mathrm{CCH}_{2}+\mathrm{H}_{2}$ & $1.5 \cdot 10^{-11}$ & Yung et al., 1984 \\
\hline 138 & $\mathrm{C}_{3} \mathrm{H}_{5}+\mathrm{H} \rightarrow \mathrm{CH}_{3} \mathrm{C}_{2} \mathrm{H}+\mathrm{H}_{2}$ & $1.5 \cdot 10^{-11}$ & Yung et al., 1984 \\
\hline 139 & $\mathrm{C}_{3} \mathrm{H}_{5}+\mathrm{H} \rightarrow \mathrm{CH}_{4}+\mathrm{C}_{2} \mathrm{H}_{2}$ & $1.5 \cdot 10^{-11}$ & Yung et al., 1984 \\
\hline 140 & $\mathrm{C}_{3} \mathrm{H}_{6}+\mathrm{H}+\mathrm{M} \rightarrow \mathrm{C}_{3} \mathrm{H}_{7}+\mathrm{M}$ & $k_{0}=2.15 \cdot 10^{-29} \times \mathrm{e}^{-349 / \mathrm{T}}$ & Pavlov et al., 2001 \\
\hline & & $k_{\infty}=4.95 \cdot 10^{-11} \times \mathrm{e}^{-1051 / \mathrm{T}}$ & Assumed same as $k\left(\mathrm{C}_{2} \mathrm{H}_{4}+\mathrm{H}\right)$ \\
\hline 141 & $\mathrm{C}_{3} \mathrm{H}_{6}+\mathrm{O} \rightarrow \mathrm{CH}_{3}+\mathrm{CH}_{3} \mathrm{CO}$ & $4.1 \cdot 10^{-12} \times \mathrm{e}^{-38 / \mathrm{T}}$ & Hampson and Garvin, 1977 \\
\hline 142 & $\mathrm{C}_{3} \mathrm{H}_{6}+\mathrm{OH} \rightarrow \mathrm{CH}_{3} \mathrm{CHO}+\mathrm{CH}_{3}$ & $4.1 \cdot 10^{-12} \times \mathrm{e}^{540 / \mathrm{T}}$ & Hampson and Garvin, 1977 \\
\hline 143 & $\mathrm{C}_{3} \mathrm{H}_{7}+\mathrm{CH}_{3} \rightarrow \mathrm{C}_{3} \mathrm{H}_{6}+\mathrm{CH}_{4}$ & $2.5 \cdot 10^{-12} \times \mathrm{e}^{-200 / \mathrm{T}}$ & Yung et al., 1984 \\
\hline 144 & $\mathrm{C}_{3} \mathrm{H}_{7}+\mathrm{H} \rightarrow \mathrm{CH}_{3}+\mathrm{C}_{2} \mathrm{H}_{5}$ & $7.95 \cdot 10^{-11} \times \mathrm{e}^{-127 / \mathrm{T}}$ & Pavlov et al., 2001 \\
\hline 145 & $\mathrm{C}_{3} \mathrm{H}_{7}+\mathrm{O} \rightarrow \mathrm{C}_{2} \mathrm{H}_{5} \mathrm{CHO}+\mathrm{H}$ & $1.1 \cdot 10^{-10}$ & Pavlov et al., 2001 \\
\hline 146 & $\mathrm{C}_{3} \mathrm{H}_{7}+\mathrm{OH} \rightarrow \mathrm{C}_{2} \mathrm{H}_{5} \mathrm{CHO}+\mathrm{H}_{2}$ & $1.1 \cdot 10^{-10}$ & Pavlov et al., 2001 \\
\hline 147 & $\mathrm{C}_{3} \mathrm{H}_{8}+\mathrm{O}+\mathrm{M} \rightarrow \mathrm{C}_{3} \mathrm{H}_{7}+\mathrm{OH}+\mathrm{M}$ & $\begin{array}{l}k_{0}=1.6 \cdot 10^{-11} \times \mathrm{e}^{-2900 / \mathrm{T}} \\
k_{\infty}=2.2 \cdot 10^{-11} \times \mathrm{e}^{-2200 / \mathrm{T}}\end{array}$ & Hampson and Garvin, 1977 \\
\hline 148 & $\mathrm{C}_{3} \mathrm{H}_{8}+\mathrm{O}^{1} \mathrm{D} \rightarrow \mathrm{C}_{3} \mathrm{H}_{7}+\mathrm{OH}$ & $1.4 \cdot 10^{-10}$ & Pavlov et al., 2001 \\
\hline 149 & $\mathrm{C}_{3} \mathrm{H}_{8}+\mathrm{OH} \rightarrow \mathrm{C}_{3} \mathrm{H}_{7}+\mathrm{H}_{2} \mathrm{O}$ & $8.6 \cdot 10^{-12} \times \mathrm{e}^{-615 / \mathrm{T}}$ & Sander et al., 2006 \\
\hline 150 & $\mathrm{CH}+\mathrm{C}_{2} \mathrm{H}_{2}+\mathrm{M} \rightarrow \mathrm{C}_{3} \mathrm{H}_{2}+\mathrm{H}+\mathrm{M}$ & $\begin{array}{l}k_{0}=2.15 \cdot 10^{-29} \times \mathrm{e}^{-349 / \mathrm{T}} \\
k_{\infty}=4.95 \cdot 10^{-11} \times \mathrm{e}^{-1051 / \mathrm{T}}\end{array}$ & Romani et al., 1993 \\
\hline 151 & $\mathrm{CH}+\mathrm{C}_{2} \mathrm{H}_{4}+\mathrm{M} \rightarrow \mathrm{CH}_{2} \mathrm{CCH}_{2}+\mathrm{H}+\mathrm{M}$ & $\begin{array}{l}k_{0}=1.75 \cdot 10^{-10} \times \mathrm{e}^{61 / \mathrm{T}} \\
k_{\infty}=5.3 \cdot 10^{-10}\end{array}$ & Romani et al., 1993 \\
\hline 152 & $\mathrm{CH}+\mathrm{C}_{2} \mathrm{H}_{4}+\mathrm{M} \rightarrow \mathrm{CH}_{3} \mathrm{C}_{2} \mathrm{H}+\mathrm{H}+\mathrm{M}$ & $\begin{array}{l}k_{0}=1.75 \cdot 10^{-10} \times \mathrm{e}^{61 / \mathrm{T}} \\
k_{\infty}=5.3 \cdot 10^{-10}\end{array}$ & Romani et al., 1993 \\
\hline 153 & $\mathrm{CH}+\mathrm{CH}_{4}+\mathrm{M} \rightarrow \mathrm{C}_{2} \mathrm{H}_{4}+\mathrm{H}+\mathrm{M}$ & $\begin{array}{l}k_{0}=2.5 \cdot 10^{-11} \times \mathrm{e}^{200 / \mathrm{T}} \\
k_{\infty}=1.7 \cdot 10^{-10}\end{array}$ & Romani et al., 1993 \\
\hline 154 & $\mathrm{CH}+\mathrm{CO}_{2} \rightarrow \mathrm{HCO}+\mathrm{CO}$ & $5.9 \cdot 10^{-12} \times \mathrm{e}^{-350 / \mathrm{T}}$ & Berman et al., 1982 \\
\hline 155 & $\mathrm{CH}+\mathrm{H} \rightarrow \mathrm{C}+\mathrm{H}_{2}$ & $1.4 \cdot 10^{-11}$ & Becker et al., 1989 \\
\hline 156 & $\mathrm{CH}+\mathrm{H}_{2} \rightarrow \mathrm{CH}_{2}^{3}+\mathrm{H}$ & $2.38 \cdot 10^{-10} \times \mathrm{e}^{-1760 / \mathrm{T}}$ & Zabarnick et al., 1986 \\
\hline 157 & $\mathrm{CH}+\mathrm{H}_{2}+\mathrm{M} \rightarrow \mathrm{CH}_{3}+\mathrm{M}$ & $\begin{array}{l}k_{0}=8.75 \cdot 10^{-31} \times \mathrm{e}^{524 / \mathrm{T}} \\
k_{\infty}=8.3 \cdot 10^{-11}\end{array}$ & Romani et al., 1993 \\
\hline 158 & $\mathrm{CH}+\mathrm{O} \rightarrow \mathrm{CO}+\mathrm{H}$ & $9.5 \cdot 10^{-11}$ & Messing et al., 1981 \\
\hline
\end{tabular}


TABle 1. (Continued)

\begin{tabular}{|c|c|c|c|}
\hline Rxn. \# & Reaction & Reaction rate constant & Reference \\
\hline 159 & $\mathrm{CH}+\mathrm{O}_{2} \rightarrow \mathrm{CO}+\mathrm{OH}$ & $5.9 \cdot 10^{-11}$ & Butler et al., 1981 \\
\hline 160 & $\mathrm{CH}_{2}^{1}+\mathrm{CH}_{4} \rightarrow \mathrm{CH}_{3}+\mathrm{CH}_{3}$ & $7.14 \cdot 10^{-12} \times \mathrm{e}^{-5050 / \mathrm{T}}$ & Böhland et al., 1985 \\
\hline 161 & $\mathrm{CH}_{2}^{1}+\mathrm{CO}_{2} \rightarrow \mathrm{H}_{2} \mathrm{CO}+\mathrm{CO}$ & $1 \cdot 10^{-12}$ & Zahnle, 1986 \\
\hline 162 & $\mathrm{CH}_{2}^{1}+\mathrm{H}_{2} \rightarrow \mathrm{CH}_{2}^{3}+\mathrm{H}_{2}$ & $1.26 \cdot 10^{-11}$ & Romani et al., 1993 \\
\hline 163 & $\mathrm{CH}_{2}^{1}+\mathrm{H}_{2} \rightarrow \mathrm{CH}_{3}+\mathrm{H}$ & $5 \cdot 10^{-15}$ & Tsang and Hampson, 1986 \\
\hline 164 & $\mathrm{CH}_{2}^{1}+\mathrm{M} \rightarrow \mathrm{CH}_{2}^{3}+\mathrm{M}$ & $8.8 \cdot 10^{-12}$ & Ashfold et al., 1981 \\
\hline 165 & $\mathrm{CH}_{2}^{1}+\mathrm{O}_{2} \rightarrow \mathrm{HCO}+\mathrm{OH}$ & $3 \cdot 10^{-11}$ & Ashfold et al., 1981 \\
\hline 166 & $\mathrm{CH}_{2}^{3}+\mathrm{C}_{2} \mathrm{H}_{2}+\mathrm{M} \rightarrow \mathrm{CH}_{2} \mathrm{CCH}_{2}+\mathrm{M}$ & $\begin{array}{l}k_{0}=3.8 \cdot 10^{-25} \\
k_{\infty}=3.7 \cdot 10^{-12}\end{array}$ & Laufer, 1981; Laufer et al., 1983 \\
\hline 167 & $\mathrm{CH}_{2}^{3}+\mathrm{C}_{2} \mathrm{H}_{2}+\mathrm{M} \rightarrow \mathrm{CH}_{3} \mathrm{C}_{2} \mathrm{H}+\mathrm{M}$ & $\begin{array}{l}k_{0}=3.8 \cdot 10^{-25} \\
k_{\infty}=2.2 \cdot 10^{-12}\end{array}$ & Laufer, 1981; Laufer et al., 1983 \\
\hline 168 & $\mathrm{CH}_{2}^{3}+\mathrm{C}_{2} \mathrm{H}_{3} \rightarrow \mathrm{CH}_{3}+\mathrm{C}_{2} \mathrm{H}_{2}$ & $3 \cdot 10^{-11}$ & Tsang and Hampson, 1986 \\
\hline 169 & $\mathrm{CH}_{2}^{3}+\mathrm{C}_{2} \mathrm{H}_{5} \rightarrow \mathrm{CH}_{3}+\mathrm{C}_{2} \mathrm{H}_{4}$ & $3 \cdot 10^{-11}$ & Tsang and Hampson, 1986 \\
\hline 170 & $\mathrm{CH}_{2}^{3}+\mathrm{CH}_{3} \rightarrow \mathrm{C}_{2} \mathrm{H}_{4}+\mathrm{H}$ & $7 \cdot 10^{-11}$ & Tsang and Hampson, 1986 \\
\hline 171 & $\mathrm{CH}_{2}^{3}+\mathrm{CO}+\mathrm{M} \rightarrow \mathrm{CH}_{2} \mathrm{CO}+\mathrm{M}$ & $\begin{array}{l}k_{0}=1.0 \cdot 10^{-28} \\
k_{\infty}=1.0 \cdot 10^{-15}\end{array}$ & Yung et al., 1984 \\
\hline 172 & $\mathrm{CH}_{2}^{3}+\mathrm{CO}_{2} \rightarrow \mathrm{H}_{2} \mathrm{CO}+\mathrm{CO}$ & $3.9 \cdot 10^{-14}$ & Laufer, 1981 \\
\hline 173 & $\mathrm{CH}_{2}^{3}+\mathrm{H} \rightarrow \mathrm{CH}+\mathrm{H}_{2}$ & $4.7 \cdot 10^{-10} \times \mathrm{e}^{-370 / \mathrm{T}}$ & Zabarnick et al., 1986 \\
\hline 174 & $\mathrm{CH}_{2}^{3}+\mathrm{H}+\mathrm{M} \rightarrow \mathrm{CH}_{3}+\mathrm{M}$ & $\begin{array}{l}k_{0}=3.1 \cdot 10^{-30} \times \mathrm{e}^{457 / \mathrm{T}} \\
k_{\infty}=1.5 \cdot 10^{-10}\end{array}$ & Gladstone et al., 1996 \\
\hline 175 & $\mathrm{CH}_{2}^{3}+\mathrm{O} \rightarrow \mathrm{CH}+\mathrm{OH}$ & $8 \cdot 10^{-12}$ & Huebner and Giguere, 1980 \\
\hline 176 & $\mathrm{CH}_{2}^{3}+\mathrm{O} \rightarrow \mathrm{CO}+\mathrm{HH}$ & $8.3 \cdot 10^{-11}$ & Homann and Wellmann, 1983 \\
\hline 177 & $\mathrm{CH}_{2}^{3}+\mathrm{O} \rightarrow \mathrm{HCO}+\mathrm{H}$ & $1 \cdot 10^{-11}$ & Huebner and Giguere, 1980 \\
\hline 178 & $\mathrm{CH}_{2}^{3}+\mathrm{O}_{2} \rightarrow \mathrm{HCO}+\mathrm{OH}$ & $4.1 \cdot 10^{-11} \times \mathrm{e}^{-750 / \mathrm{T}}$ & Baulch et al., 1994 \\
\hline 179 & $\mathrm{CH}_{2} \mathrm{CCH}_{2}+\mathrm{H} \rightarrow \mathrm{C}_{3} \mathrm{H}_{5}$ & $\begin{array}{l}k_{0}=8.9 \cdot 10^{-29} \times \mathrm{e}^{-1225 / \mathrm{T}} \times(\mathrm{T} / 300)^{-2.0} \\
k_{\infty}=1.4 \cdot 10^{-11} \times \mathrm{e}^{-1000 / \mathrm{T}}\end{array}$ & Yung et al., 1984 \\
\hline 180 & $\mathrm{CH}_{2} \mathrm{CCH}_{2}+\mathrm{H} \rightarrow \mathrm{CH}_{3}+\mathrm{C}_{2} \mathrm{H}_{2}$ & $\begin{array}{l}k_{0}=8.9 \cdot 10^{-29} \times \mathrm{e}^{-1225 / \mathrm{T}} \times(\mathrm{T} / 300)^{-2.0} \\
k_{\infty}=9.7 \cdot 10^{-13} \times \mathrm{e}^{-1550 / \mathrm{T}}\end{array}$ & Yung et al., 1984 \\
\hline 181 & $\mathrm{CH}_{2} \mathrm{CCH}_{2}+\mathrm{H} \rightarrow \mathrm{CH}_{3} \mathrm{C}_{2} \mathrm{H}+\mathrm{H}$ & $1 \cdot 10^{-11} \times \mathrm{e}^{-1000 / \mathrm{T}}$ & Yung et al., 1984 \\
\hline 182 & $\mathrm{CH}_{2} \mathrm{CO}+\mathrm{H} \rightarrow \mathrm{CH}_{3}+\mathrm{CO}$ & $1.9 \cdot 10^{-11} \times \mathrm{e}^{-1725 / \mathrm{T}}$ & Michael et al., 1979 \\
\hline 183 & $\mathrm{CH}_{2} \mathrm{CO}+\mathrm{O} \rightarrow \mathrm{H}_{2} \mathrm{CO}+\mathrm{CO}$ & $3.3 \cdot 10^{-11}$ & Lee, 1980; Miller et al., 1982 \\
\hline 184 & $\mathrm{CH}_{3}+\mathrm{C}_{2} \mathrm{H}_{3} \rightarrow \mathrm{C}_{3} \mathrm{H}_{5}+\mathrm{H}$ & $2.4 \cdot 10^{-13}$ & Romani et al., 1993 \\
\hline 185 & $\mathrm{CH}_{3}+\mathrm{CH}_{3}+\mathrm{M} \rightarrow \mathrm{C}_{2} \mathrm{H}_{6}+\mathrm{M}$ & $\begin{array}{l}k_{0}=4.0 \cdot 10^{-24} \times \mathrm{e}^{-1390 / \mathrm{T}} \times(\mathrm{T} / 300)^{-7.0} \\
k_{\infty}=1.79 \cdot 10^{-10} \times \mathrm{e}^{-329 / \mathrm{T}}\end{array}$ & Wagner and Wardlaw, 1988 \\
\hline 186 & $\mathrm{CH}_{3}+\mathrm{CO}+\mathrm{M} \rightarrow \mathrm{CH}_{3} \mathrm{CO}+\mathrm{M}$ & $1.4 \cdot 10^{-32} \times \mathrm{e}^{-3000 / \mathrm{T}} \times \mathrm{den}$ & Watkins and Word, 1974 \\
\hline 187 & $\mathrm{CH}_{3}+\mathrm{H}+\mathrm{M} \rightarrow \mathrm{CH}_{4}+\mathrm{M}$ & $k_{0}=6.0 \cdot 10^{-28} \times(\mathrm{T} / 298)^{-1.80}$ & $\begin{array}{l}\text { Baulch et al., 1994; } \\
\text { Tsang and Hampson, } 1986\end{array}$ \\
\hline & & $k_{\infty}=2.0 \cdot 10^{-10} \times(\mathrm{T} / 298)^{-0.40}$ & \\
\hline 188 & $\mathrm{CH}_{3}+\mathrm{H}_{2} \mathrm{CO} \rightarrow \mathrm{CH}_{4}+\mathrm{HCO}$ & $1.60 \cdot 10^{-16} \times \mathrm{e}^{899 / \mathrm{T}} \times(\mathrm{T} / 298)^{6.10}$ & Baulch et al., 1994 \\
\hline 189 & $\mathrm{CH}_{3}+\mathrm{HCO} \rightarrow \mathrm{CH}_{4}+\mathrm{CO}$ & $2.01 \cdot 10^{-10}$ & Tsang and Hampson, 1986 \\
\hline 190 & $\mathrm{CH}_{3}+\mathrm{HNO} \rightarrow \mathrm{CH}_{4}+\mathrm{NO}$ & $1.85 \cdot 10^{-11} \times \mathrm{e}^{-176 / \mathrm{T}} \times(\mathrm{T} / 298)^{0.6}$ & Choi and Lin, 2005 \\
\hline 191 & $\mathrm{CH}_{3}+\mathrm{O} \rightarrow \mathrm{H}_{2} \mathrm{CO}+\mathrm{H}$ & $1.1 \cdot 10^{-10}$ & Sander et al., 2006 \\
\hline 192 & $\mathrm{CH}_{3}+\mathrm{O}_{2} \rightarrow \mathrm{H}_{2} \mathrm{CO}+\mathrm{OH}$ & $\begin{array}{l}k_{0}=4.0 \cdot 10^{-31} \times(\mathrm{T} / 300)^{-3.6} \\
k_{\infty}=1.2 \cdot 10^{-12} \times(\mathrm{T} / 300)^{-1.1}\end{array}$ & Sander et al., 2006 \\
\hline 193 & $\mathrm{CH}_{3}+\mathrm{O}_{3} \rightarrow \mathrm{H}_{2} \mathrm{CO}+\mathrm{HO}_{2}$ & $5.4 \cdot 10^{-12} \times \mathrm{e}^{-220 / \mathrm{T}}$ & Sander et al., 2006 \\
\hline 194 & $\mathrm{CH}_{3}+\mathrm{OH} \rightarrow \mathrm{CH}_{3} \mathrm{O}+\mathrm{H}$ & $9.3 \cdot 10^{-11} \times \mathrm{e}^{-1606 / \mathrm{T}} \times(\mathrm{T} / 298)$ & Jasper et al., 2007 \\
\hline 195 & $\mathrm{CH}_{3}+\mathrm{OH} \rightarrow \mathrm{CO}+\mathrm{H}_{2}+\mathrm{H}_{2}$ & $6.7 \cdot 10^{-12}$ & Fenimore, 1969 \\
\hline 196 & $\mathrm{CH}_{3} \mathrm{C}_{2} \mathrm{H}+\mathrm{H}+\mathrm{M} \rightarrow \mathrm{C}_{3} \mathrm{H}_{5}+\mathrm{M}$ & $\begin{array}{l}k_{0}=8.88 \cdot 10^{-29} \times \mathrm{e}^{-1225 / \mathrm{T}} \times(\mathrm{T} / 300)^{-2} \\
k_{\infty}=9.7 \cdot 10^{-12} \times \mathrm{e}^{-1550 / \mathrm{T}}\end{array}$ & Yung et al., 1984 \\
\hline 197 & $\mathrm{CH}_{3} \mathrm{C}_{2} \mathrm{H}+\mathrm{H} \rightarrow \mathrm{CH}_{3}+\mathrm{C}_{2} \mathrm{H}_{2}$ & $\begin{array}{l}k_{0}=8.88 \cdot 10^{-29} \times \mathrm{e}^{-1225 / \mathrm{T}} \times(\mathrm{T} / 300)^{-2} \\
k_{\infty}=9.7 \cdot 10^{-12} \times \mathrm{e}^{-1550 / \mathrm{T}}\end{array}$ & Whytock et al., 1976 \\
\hline 198 & $\mathrm{CH}_{3} \mathrm{CHO}+\mathrm{CH}_{3} \rightarrow \mathrm{CH}_{3} \mathrm{CO}+\mathrm{CH}_{4}$ & $2.8 \cdot 10^{-11} \times \mathrm{e}^{-1540 / \mathrm{T}}$ & Zahnle, 1986 \\
\hline 199 & $\mathrm{CH}_{3} \mathrm{CHO}+\mathrm{H} \rightarrow \mathrm{CH}_{3} \mathrm{CO}+\mathrm{H}_{2}$ & $2.8 \cdot 10^{-11} \times \mathrm{e}^{-1540 / \mathrm{T}}$ & Zahnle, 1986 \\
\hline 200 & $\mathrm{CH}_{3} \mathrm{CHO}+\mathrm{O} \rightarrow \mathrm{CH}_{3} \mathrm{CO}+\mathrm{OH}$ & $5.8 \cdot 10^{-13}$ & Washida, 1981 \\
\hline 201 & $\mathrm{CH}_{3} \mathrm{CHO}+\mathrm{OH} \rightarrow \mathrm{CH}_{3} \mathrm{CO}+\mathrm{H}_{2} \mathrm{O}$ & $1.6 \cdot 10^{-11}$ & Niki et al., 1978 \\
\hline 202 & $\mathrm{CH}_{3} \mathrm{CO}+\mathrm{CH}_{3} \rightarrow \mathrm{C}_{2} \mathrm{H}_{6}+\mathrm{CO}$ & $5.4 \cdot 10^{-11}$ & Adachi et al., 1981 \\
\hline 203 & $\mathrm{CH}_{3} \mathrm{CO}+\mathrm{CH}_{3} \rightarrow \mathrm{CH}_{4}+\mathrm{CH}_{2} \mathrm{CO}$ & $8.6 \cdot 10^{-11}$ & Adachi et al., 1981 \\
\hline 204 & $\mathrm{CH}_{3} \mathrm{CO}+\mathrm{H} \rightarrow \mathrm{CH}_{4}+\mathrm{CO}$ & $1 \cdot 10^{-10}$ & Zahnle, 1986 \\
\hline 205 & $\mathrm{CH}_{3} \mathrm{CO}+\mathrm{O} \rightarrow \mathrm{H}_{2} \mathrm{CO}+\mathrm{HCO}$ & $5 \cdot 10^{-11}$ & Zahnle, 1986 \\
\hline 206 & $\mathrm{CH}_{3} \mathrm{O}+\mathrm{CO} \rightarrow \mathrm{CH}_{3}+\mathrm{CO}_{2}$ & $2.6 \cdot 10^{-11} \times \mathrm{e}^{-5940 / \mathrm{T}}$ & Wen et al., 1989 \\
\hline 207 & $\mathrm{CH}_{3} \mathrm{O}_{2}+\mathrm{H} \rightarrow \mathrm{CH}_{4}+\mathrm{O}_{2}$ & $1.6 \cdot 10^{-10}$ & Tsang and Hampson, 1986 \\
\hline 208 & $\mathrm{CH}_{3} \mathrm{O}_{2}+\mathrm{H} \rightarrow \mathrm{H}_{2} \mathrm{O}+\mathrm{H}_{2} \mathrm{CO}$ & $1 \cdot 10^{-11}$ & Zahnle et al., 2006 \\
\hline
\end{tabular}


TAble 1. (Continued)

\begin{tabular}{|c|c|c|c|}
\hline Rxn. \# & Reaction & Reaction rate constant & Reference \\
\hline 209 & $\mathrm{CH}_{3} \mathrm{O}_{2}+\mathrm{O} \rightarrow \mathrm{H}_{2} \mathrm{CO}+\mathrm{HO}_{2}$ & $1 \cdot 10^{-11}$ & Vaghjiani and Ravishankara, 1990 \\
\hline 210 & $\mathrm{CH}_{4}+\mathrm{HS} \rightarrow \mathrm{CH}_{3}+\mathrm{H}_{2} \mathrm{~S}$ & $2.99 \cdot 10^{-31}$ & Kerr and Trotman-Dickenson, 1957 \\
\hline 211 & $\mathrm{CH}_{4}+\mathrm{O} \rightarrow \mathrm{CH}_{3}+\mathrm{OH}$ & $8.75 \cdot 10^{-12} \times \mathrm{e}^{-4330 / \mathrm{T}} \times(\mathrm{T} / 298)^{1.5}$ & Tsang and Hampson, 1986 \\
\hline 212 & $\mathrm{CH}_{4}+\mathrm{O}^{1} \mathrm{D} \rightarrow \mathrm{CH}_{3}+\mathrm{OH}$ & $1.28 \cdot 10^{-10}$ & Sander et al., 2006 \\
\hline 213 & $\mathrm{CH}_{4}+\mathrm{O}^{1} \mathrm{D} \rightarrow \mathrm{H}_{2} \mathrm{CO}+\mathrm{H}_{2}$ & $2.25 \cdot 10^{-11}$ & Sander et al., 2006 \\
\hline 214 & $\mathrm{CH}_{4}+\mathrm{OH} \rightarrow \mathrm{CH}_{3}+\mathrm{H}_{2} \mathrm{O}$ & $2.45 \cdot 10^{-12} \times \mathrm{e}^{-1775 / \mathrm{T}}$ & Sander et al., 2006 \\
\hline 215 & $\mathrm{CO}+\mathrm{O}+\mathrm{M} \rightarrow \mathrm{CO}_{2}+\mathrm{M}$ & $1.7 \cdot 10^{-33} \times \mathrm{e}^{-1515 / \mathrm{T}} \times$ den & Tsang and Hampson, 1986 \\
\hline 216 & $\mathrm{CO}+\mathrm{OH} \rightarrow \mathrm{CO}_{2}+\mathrm{H}$ & $1.5 \cdot 10^{-13} \times(1+0.6 \times$ den $)$ & Sander et al., 2006 \\
\hline 217 & $\mathrm{H}+\mathrm{CO}+\mathrm{M} \rightarrow \mathrm{HCO}+\mathrm{M}$ & $5.29 \cdot 10^{-34} \times \mathrm{e}^{-100 / \mathrm{T}} \times$ den & Baulch et al., 1994 \\
\hline 218 & $\mathrm{H}+\mathrm{H}+\mathrm{M} \rightarrow \mathrm{H}_{2}+\mathrm{M}$ & $8.85 \cdot 10^{-33} \times(\mathrm{T} / 298)^{-0.6} \times$ den & Baulch et al., 1994 \\
\hline 219 & $\mathrm{H}+\mathrm{HCO} \rightarrow \mathrm{H}_{2}+\mathrm{CO}$ & $1.5 \cdot 10^{-10}$ & Baulch et al., 1992 \\
\hline 220 & $\mathrm{H}+\mathrm{HNO} \rightarrow \mathrm{H}_{2}+\mathrm{NO}$ & $3.01 \cdot 10^{-11} \times \mathrm{e}^{500 / \mathrm{T}}$ & Tsang and Herron, 1991 \\
\hline 221 & $\mathrm{H}+\mathrm{HO}_{2} \rightarrow \mathrm{H}_{2}+\mathrm{O}_{2}$ & $6.9 \cdot 10^{-12}$ & Sander et al., 2006 \\
\hline 222 & $\mathrm{H}+\mathrm{HO}_{2} \rightarrow \mathrm{H}_{2} \mathrm{O}+\mathrm{O}$ & $1.62 \cdot 10^{-12}$ & Sander et al., 2006 \\
\hline 223 & $\mathrm{H}+\mathrm{HO}_{2} \rightarrow \mathrm{OH}+\mathrm{OH}$ & $7.29 \cdot 10^{-11}$ & Sander et al., 2006 \\
\hline 224 & $\mathrm{H}+\mathrm{NO}+\mathrm{M} \rightarrow \mathrm{HNO}+\mathrm{M}$ & $2.1 \cdot 10^{-32} \times(\mathrm{T} / 298)^{1.00} \times$ den & Hampson and Garvin, 1977 \\
\hline 225 & $\mathrm{H}+\mathrm{O}_{2}+\mathrm{M} \rightarrow \mathrm{HO}_{2}+\mathrm{M}$ & $5.7 \cdot 10^{-32} \times 7.5 \cdot 10^{-11} \times(\mathrm{T} / 298)^{1.6}$ & Sander et al., 2006 \\
\hline 226 & $\mathrm{H}+\mathrm{O}_{3} \rightarrow \mathrm{OH}+\mathrm{O}_{2}$ & $1.4 \cdot 10^{-10} \times \mathrm{e}^{-470 / \mathrm{T}}$ & Sander et al., 2006 \\
\hline 227 & $\mathrm{H}+\mathrm{OH}+\mathrm{M} \rightarrow \mathrm{H}_{2} \mathrm{O}+\mathrm{M}$ & $6.8 \cdot 10^{-31} \times(\mathrm{T} / 300)^{-2} \times$ den & McEwan and Phillips, 1975 \\
\hline 228 & $\mathrm{H}+\mathrm{SO}+\mathrm{M} \rightarrow \mathrm{HSO}+\mathrm{M}$ & $\begin{array}{l}k_{0}=5.7 \cdot 10^{-32} \times(\mathrm{T} / 298)^{1.6} \\
k_{\infty}=7.5 \cdot 10^{-11}\end{array}$ & Kasting, 1990 \\
\hline 229 & $\mathrm{H}_{2}+\mathrm{O} \rightarrow \mathrm{OH}+\mathrm{H}$ & $1.34 \cdot 10^{-15} \times \mathrm{e}^{-1460 / \mathrm{T}} \times(\mathrm{T} / 298)^{6.52}$ & Robie et al., 1990 \\
\hline 230 & $\mathrm{H}_{2}+\mathrm{O}^{1} \mathrm{D} \rightarrow \mathrm{OH}+\mathrm{H}$ & $1.1 \cdot 10^{-11}$ & Sander et al., 2006 \\
\hline 231 & $\mathrm{H}_{2}+\mathrm{OH} \rightarrow \mathrm{H}_{2} \mathrm{O}+\mathrm{H}$ & $5.5 \cdot 10^{-12} \times \mathrm{e}^{-2000 / \mathrm{T}}$ & Sander et al., 2006 \\
\hline 232 & $\mathrm{H}_{2} \mathrm{CO}+\mathrm{H} \rightarrow \mathrm{H}_{2}+\mathrm{HCO}$ & $2.14^{-12} \times \mathrm{e}^{-1090 / \mathrm{T}} \times(\mathrm{T} / 298)^{1.62}$ & Baulch et al., 1994 \\
\hline 233 & $\mathrm{H}_{2} \mathrm{CO}+\mathrm{O} \rightarrow \mathrm{HCO}+\mathrm{OH}$ & $3.4 \cdot 10^{-11} \times \mathrm{e}^{-1600 / \mathrm{T}}$ & Sander et al., 2006 \\
\hline 234 & $\mathrm{H}_{2} \mathrm{CO}+\mathrm{OH} \rightarrow \mathrm{H}_{2} \mathrm{O}+\mathrm{HCO}$ & $5.5 \cdot 10^{-12} \times \mathrm{e}^{125 / \mathrm{T}}$ & Sander et al., 2006 \\
\hline 235 & $\mathrm{H}_{2} \mathrm{O}+\mathrm{O}^{1} \mathrm{D} \rightarrow \mathrm{OH}+\mathrm{OH}$ & $2.2 \cdot 10^{-10}$ & Sander et al., 2006 \\
\hline 236 & $\mathrm{H}_{2} \mathrm{O}_{2}+\mathrm{O} \rightarrow \mathrm{OH}+\mathrm{HO}_{2}$ & $1.4 \cdot 10^{-12} \times \mathrm{e}^{-2000 / \mathrm{T}}$ & Sander et al., 2006 \\
\hline 237 & $\mathrm{H}_{2} \mathrm{O}_{2}+\mathrm{OH} \rightarrow \mathrm{HO}_{2}+\mathrm{H}_{2} \mathrm{O}$ & $2.9 \cdot 10^{-12} \times \mathrm{e}^{-160 / \mathrm{T}}$ & Sander et al., 2006 \\
\hline 238 & $\mathrm{H}_{2} \mathrm{~S}+\mathrm{H} \rightarrow \mathrm{H}_{2}+\mathrm{HS}$ & $3.66 \cdot 10^{-12} \times \mathrm{e}^{-455 / \mathrm{T}} \times(\mathrm{T} / 298)^{1.94}$ & Peng et al., 1999 \\
\hline 239 & $\mathrm{H}_{2} \mathrm{~S}+\mathrm{O} \rightarrow \mathrm{OH}+\mathrm{HS}$ & $9.2 \cdot 10^{-12} \times \mathrm{e}^{-1800 / \mathrm{T}}$ & Sander et al., 2006 \\
\hline 240 & $\mathrm{H}_{2} \mathrm{~S}+\mathrm{OH} \rightarrow \mathrm{H}_{2} \mathrm{O}+\mathrm{HS}$ & $6.0 \cdot 10^{-12} \times \mathrm{e}^{-70 / \mathrm{T}}$ & Sander et al., 2006 \\
\hline 241 & $\mathrm{HCO}+\mathrm{H}+\mathrm{M} \rightarrow \mathrm{CO}+\mathrm{M}$ & $6.0 \cdot 10^{-11} \times \mathrm{e}^{-7721 / \mathrm{T}} \times$ den & Krasnoperov et al., 2004 \\
\hline 242 & $\mathrm{HCO}+\mathrm{H}_{2} \mathrm{CO} \rightarrow \mathrm{CH}_{3} \mathrm{O}+\mathrm{CO}$ & $3.8 \cdot 10^{-17}$ & Wen et al., 1989 \\
\hline 243 & $\mathrm{HCO}+\mathrm{HCO} \rightarrow \mathrm{H}_{2} \mathrm{CO}+\mathrm{CO}$ & $3.0 \cdot 10^{-11}$ & Tsang and Hampson, 1986 \\
\hline 244 & $\mathrm{HCO}+\mathrm{NO} \rightarrow \mathrm{HNO}+\mathrm{CO}$ & $1.2 \cdot 10^{-11}$ & Tsang and Hampson, 1986 \\
\hline 245 & $\mathrm{HCO}+\mathrm{O}_{2} \rightarrow \mathrm{HO}_{2}+\mathrm{CO}$ & $5.2 \cdot 10^{-12}$ & Sander et al., 2006 \\
\hline 246 & $\mathrm{HNO}+\mathrm{NO}+\mathrm{M} \rightarrow \mathrm{H}+\mathrm{M}$ & $1.04 \cdot 10^{-6} \times \mathrm{e}^{25618 / \mathrm{T}} \times(\mathrm{T} / 298)^{-1.61} \times \operatorname{den}$ & Tsang and Hampson, 1986 \\
\hline 247 & $\mathrm{HNO}_{2}+\mathrm{OH} \rightarrow \mathrm{H}_{2} \mathrm{O}+\mathrm{NO}_{2}$ & $1.8 \cdot 10^{-11} \times \mathrm{e}^{-390 / \mathrm{T}}$ & Sander et al., 2006 \\
\hline 248 & $\mathrm{HNO}_{3}+\mathrm{OH} \rightarrow \mathrm{H}_{2} \mathrm{O}+\mathrm{NO}_{2}+\mathrm{O}$ & $\begin{array}{l}7.2 \cdot 10^{-15} \times \mathrm{e}^{-785 / \mathrm{T}}+ \\
\left(1.9 \cdot 10^{-33} \times \mathrm{e}^{725 / \mathrm{T}} \times \mathrm{den}\right) / \\
\left(1+4.6 \cdot 10^{-16} \times \mathrm{e}^{-715 / \mathrm{T}} \times \mathrm{den}\right)\end{array}$ & Sander et al., 2006 \\
\hline 249 & $\mathrm{HO}_{2}+\mathrm{HO}_{2} \rightarrow \mathrm{H}_{2} \mathrm{O}_{2}+\mathrm{O}_{2}$ & $\begin{array}{l}k_{0}=2.3 \cdot 10^{-13} \times \mathrm{e}^{590 / \mathrm{T}} \\
k_{\infty}=1.7 \cdot 10^{-33} \times \mathrm{e}^{1000 / \mathrm{T}}\end{array}$ & Sander et al., 2006 \\
\hline 250 & $\mathrm{HO}_{2}+\mathrm{O} \rightarrow \mathrm{OH}+\mathrm{O}_{2}$ & $3.0 \cdot 10^{-11} \times \mathrm{e}^{200 / \mathrm{T}}$ & Sander et al., 2006 \\
\hline 251 & $\mathrm{HO}_{2}+\mathrm{O}_{3} \rightarrow \mathrm{OH}+\mathrm{O}_{2}+\mathrm{O}_{2}$ & $1.1 \cdot 10^{-14} \times \mathrm{e}^{-490 / \mathrm{T}}$ & Sander et al., 2006 \\
\hline 252 & $\mathrm{HS}+\mathrm{H} \rightarrow \mathrm{H}_{2}+\mathrm{S}$ & $3.0 \cdot 10^{-11}$ & Schofield, 1973 \\
\hline 253 & $\mathrm{HS}+\mathrm{H}_{2} \mathrm{CO} \rightarrow \mathrm{H}_{2} \mathrm{~S}+\mathrm{HCO}$ & $1.7 \cdot 10^{-11} \times \mathrm{e}^{-800 / \mathrm{T}}$ & Sander et al., 2006 \\
\hline 254 & $\mathrm{HS}+\mathrm{HCO} \rightarrow \mathrm{H}_{2} \mathrm{~S}+\mathrm{CO}$ & $5.0 \cdot 10^{-11}$ & Kasting, 1990 \\
\hline 255 & $\mathrm{HS}+\mathrm{HO}_{2} \rightarrow \mathrm{H}_{2} \mathrm{~S}+\mathrm{O}_{2}$ & $1.0 \cdot 10^{-11}$ & Stachnik and Molina, 1987 \\
\hline 256 & $\mathrm{HS}+\mathrm{HS} \rightarrow \mathrm{H}_{2} \mathrm{~S}+\mathrm{S}$ & $1.5^{-11}$ & Schofield, 1973 \\
\hline 257 & $\mathrm{HS}+\mathrm{NO}_{2} \rightarrow \mathrm{HSO}+\mathrm{NO}$ & $2.9 \cdot 10^{-11} \times \mathrm{e}^{240 / \mathrm{T}}$ & Sander et al., 2006 \\
\hline 258 & $\mathrm{HS}+\mathrm{O} \rightarrow \mathrm{H}+\mathrm{SO}$ & $1.6 \cdot 10^{-10}$ & Sander et al., 2006 \\
\hline 259 & $\mathrm{HS}+\mathrm{O}_{2} \rightarrow \mathrm{OH}+\mathrm{SO}$ & $4.0 \cdot 10^{-19}$ & Sander et al., 2006 \\
\hline 260 & $\mathrm{HS}+\mathrm{O}_{3} \rightarrow \mathrm{HSO}+\mathrm{O}_{2}$ & $9.0 \cdot 10^{-12} \times \mathrm{e}^{-280 / \mathrm{T}}$ & Sander et al., 2006 \\
\hline 261 & $\mathrm{HS}+\mathrm{S} \rightarrow \mathrm{H}+\mathrm{S}_{2}$ & $2.2 \cdot 10^{-11} \times \mathrm{e}^{-120 / \mathrm{T}}$ & Kasting, 1990 \\
\hline 262 & $\mathrm{HSO}+\mathrm{H} \rightarrow \mathrm{H}_{2}+\mathrm{SO}$ & $6.48 \cdot 10^{-12}$ & Sander et al., 2006 \\
\hline 263 & $\mathrm{HSO}+\mathrm{H} \rightarrow \mathrm{HS}+\mathrm{OH}$ & $7.29 \cdot 10^{-11}$ & Sander et al., 2006 \\
\hline 264 & $\mathrm{HSO}+\mathrm{HS} \rightarrow \mathrm{H}_{2} \mathrm{~S}+\mathrm{SO}$ & $1 \cdot 10^{-12}$ & Kasting, 1990 \\
\hline 265 & $\mathrm{HSO}+\mathrm{NO} \rightarrow \mathrm{HNO}+\mathrm{SO}$ & $1.0 \cdot 10^{-15}$ & Atkinson et al., 2004 \\
\hline 266 & $\mathrm{HSO}+\mathrm{O} \rightarrow \mathrm{OH}+\mathrm{SO}$ & $3.0 \cdot 10^{-11} \times \mathrm{e}^{-200 / \mathrm{T}}$ & Kasting, 1990 \\
\hline
\end{tabular}


TAble 1. (Continued)

\begin{tabular}{|c|c|c|c|}
\hline Rxn. \# & Reaction & Reaction rate constant & Reference \\
\hline 267 & $\mathrm{HSO}+\mathrm{OH} \rightarrow \mathrm{H}_{2} \mathrm{O}+\mathrm{SO}$ & $5.2 \cdot 10^{-12}$ & Sander et al., 2006 \\
\hline 268 & $\mathrm{HSO}+\mathrm{S} \rightarrow \mathrm{HS}+\mathrm{SO}$ & $1 \cdot 10^{-11}$ & Kasting, 1990 \\
\hline 269 & $\mathrm{HSO}_{3}+\mathrm{H} \rightarrow \mathrm{H}_{2}+\mathrm{SO}_{3}$ & $1.0 \cdot 10^{-11}$ & Kasting, 1990 \\
\hline 270 & $\mathrm{HSO}_{3}+\mathrm{O} \rightarrow \mathrm{OH}+\mathrm{SO}_{3}$ & $1.0 \cdot 10^{-11}$ & Kasting, 1990 \\
\hline 271 & $\mathrm{HSO}_{3}+\mathrm{O}_{2} \rightarrow \mathrm{HO}_{2}+\mathrm{SO}_{3}$ & $1.3 \cdot 10^{-12} \times \mathrm{e}^{-330 / \mathrm{T}}$ & Sander et al., 2006 \\
\hline 272 & $\mathrm{HSO}_{3}+\mathrm{OH} \rightarrow \mathrm{H}_{2} \mathrm{O}+\mathrm{SO}_{3}$ & $1.0 \cdot 10^{-11}$ & Kasting, 1990 \\
\hline 273 & $\mathrm{~N}+\mathrm{NO} \rightarrow \mathrm{N}_{2}+\mathrm{O}$ & $2.1 \cdot 10^{-11} \times \mathrm{e}^{-100 / \mathrm{T}}$ & Sander et al., 2006 \\
\hline 274 & $\mathrm{~N}+\mathrm{O}_{2} \rightarrow \mathrm{NO}+\mathrm{O}$ & $1.5 \cdot 10^{-12} \times \mathrm{e}^{-3600 / \mathrm{T}}$ & Sander et al. 2006 \\
\hline 275 & $\mathrm{~N}+\mathrm{OH} \rightarrow \mathrm{NO}+\mathrm{H}$ & $3.8 \cdot 10^{-11} \times \mathrm{e}^{85 / \mathrm{T}}$ & Atkinson et al., 1989 \\
\hline 276 & $\mathrm{~N}_{2} \mathrm{H}_{3}+\mathrm{H} \rightarrow \mathrm{NH}_{2}+\mathrm{NH}_{2}$ & $2.7 \cdot 10^{-12}$ & Gehring et al., 1971 \\
\hline 277 & $\mathrm{~N}_{2} \mathrm{H}_{3}+\mathrm{N}_{2} \mathrm{H}_{3} \rightarrow \mathrm{N}_{2} \mathrm{H}_{4}+\mathrm{N}_{2}+\mathrm{H}_{2}$ & $6 \cdot 10^{-11}$ & Kuhn and Atreya, 1979 \\
\hline 278 & $\mathrm{~N}_{2} \mathrm{H}_{4}+\mathrm{H} \rightarrow \mathrm{N}_{2} \mathrm{H}_{3}+\mathrm{H}_{2}$ & $9.9 \cdot 10^{-12} \times \mathrm{e}^{-1200 / \mathrm{T}}$ & Stief and Payne, 1976 \\
\hline 279 & $\mathrm{NH}+\mathrm{H}+\mathrm{M} \rightarrow \mathrm{NH}_{2}+\mathrm{M}$ & $\left(6 \cdot 10^{-30} \times\right.$ den $) /\left(1+3 \cdot 10^{-20} \times\right.$ den $)$ & Kasting, 1982 \\
\hline 280 & $\mathrm{NH}+\mathrm{NO} \rightarrow \mathrm{N}_{2}+\mathrm{OH}$ & $4.9 \cdot 10^{-11}$ & Sander et al., 2006 \\
\hline 281 & $\mathrm{NH}+\mathrm{O} \rightarrow \mathrm{N}+\mathrm{OH}$ & $1 \cdot 10^{-11}$ & Kasting, 1982 \\
\hline 282 & $\mathrm{NH}+\mathrm{O} \rightarrow \mathrm{NH}_{2}+\mathrm{CO}$ & $1 \cdot 10^{-11}$ & Pavlov et al., 2001 \\
\hline 283 & $\mathrm{NH}_{2}+\mathrm{H}+\mathrm{M} \rightarrow \mathrm{NH}_{3}+\mathrm{M}$ & $\left(6 \cdot 10^{-30} \times\right.$ den $) /\left(1+3 \cdot 10^{-20} \times\right.$ den $)$ & Gordon et al., 1971 \\
\hline 284 & $\mathrm{NH}_{2}+\mathrm{HCO} \rightarrow \mathrm{NH}_{3}+\mathrm{CO}$ & $1 \cdot 10^{-11}$ तोeाti & Pavlov et al., 2001 \\
\hline 285 & $\mathrm{NH}_{2}+\mathrm{NH}_{2} \rightarrow \mathrm{N}_{2} \mathrm{H}_{4}$ & $1 \cdot 10^{-10}$ & Gordon et al., 1971 \\
\hline 286 & $\mathrm{NH}_{2}+\mathrm{NO} \rightarrow \mathrm{N}_{2}+\mathrm{H}_{2} \mathrm{O}$ & $3.8 \cdot 10^{-12} \times \mathrm{e}^{450 / \mathrm{T}}$ & Sander et al., 2006 \\
\hline 287 & $\mathrm{NH}_{2}+\mathrm{O} \rightarrow \mathrm{HNO}+\mathrm{H}$ & $5 \cdot 10^{-12}$ & Albers et al., 1969 \\
\hline 288 & $\mathrm{NH}_{2}+\mathrm{O} \rightarrow \mathrm{NH}+\mathrm{OH}$ & $5 \cdot 10^{-12}$ & Albers et al., 1969 \\
\hline 289 & $\mathrm{NH}_{2}^{*}+\mathrm{H}_{2} \rightarrow \mathrm{NH}_{3}+\mathrm{H}$ & $3 \cdot 10^{-11}$ & Kasting, 1982 \\
\hline 290 & $\mathrm{NH}_{2}^{*}+\mathrm{M} \rightarrow \mathrm{NH}_{2}+\mathrm{M}$ & $3 \cdot 10^{-11}$ & Kasting, 1982 \\
\hline 291 & $\mathrm{NH}_{3}+\mathrm{O}^{1} \mathrm{D} \rightarrow \mathrm{NH}_{2}+\mathrm{OH}$ & $2.5 \cdot 10^{-10}$ & Sander et al., 2006 \\
\hline 292 & $\mathrm{NH}_{3}+\mathrm{OH} \rightarrow \mathrm{NH}_{2}+\mathrm{H}_{2} \mathrm{O}$ & $1.7 \cdot 10^{-12} \times \mathrm{e}^{-710 / \mathrm{T}}$ & Sander et al., 2006 \\
\hline 293 & $\mathrm{NO}+\mathrm{HO}_{2} \rightarrow \mathrm{NO}_{2}+\mathrm{OH}$ & $3.5 \cdot 10^{-12} \times \mathrm{e}^{250 / \mathrm{T}}$ & Sander et al., 2006 \\
\hline 294 & $\mathrm{NO}+\mathrm{O}+\mathrm{M} \rightarrow \mathrm{NO}_{2}+\mathrm{M}$ & $9 \cdot 10^{-31} \times 3 \cdot 10^{-11} \times(\mathrm{T} / 298)^{1.5}$ & Sander et al., 2006 \\
\hline 295 & $\mathrm{NO}+\mathrm{O}_{3} \rightarrow \mathrm{NO}_{2}+\mathrm{O}_{2}$ & $2.0 \cdot 10^{-12} \times \mathrm{e}^{-1400 / \mathrm{T}}$ & Sander et al., 2006 \\
\hline 296 & $\mathrm{NO}+\mathrm{OH}+\mathrm{M} \rightarrow \mathrm{HNO}_{2}+\mathrm{M}$ & $\begin{array}{l}k_{0}=7 \cdot 10^{-31} \times(\mathrm{T} / 298)^{2.6} \\
k_{\infty}=3.6 \cdot 10^{-11} \times(\mathrm{T} / 298)^{0.1}\end{array}$ & Sander et al., 2006 \\
\hline 297 & $\mathrm{NO}_{2}+\mathrm{H} \rightarrow \mathrm{NO}+\mathrm{OH}$ & $4 \cdot 10^{-10} \times \mathrm{e}^{-340 / \mathrm{T}}$ & Sander et al., 2006 \\
\hline 298 & $\mathrm{NO}_{2}+\mathrm{O} \rightarrow \mathrm{NO}+\mathrm{O}_{2}$ & $5.6 \cdot 10^{-12} \times \mathrm{e}^{180 / \mathrm{T}}$ & Sander et al., 2006 \\
\hline 299 & $\mathrm{NO}_{2}+\mathrm{OH}+\mathrm{M} \rightarrow \mathrm{HNO}_{3}+\mathrm{M}$ & $\begin{array}{l}k_{0}=2.0 \cdot 10^{-30} \times(\mathrm{T} / 298)^{3.0} \\
k_{\infty}=2.5 \cdot 10^{-11}\end{array}$ & Sander et al., 2006 \\
\hline 300 & $\mathrm{O}+\mathrm{HCO} \rightarrow \mathrm{H}+\mathrm{CO}_{2}$ & $5.0 \cdot 10^{-11}$ & Tsang and Hampson, 1986 \\
\hline 301 & $\mathrm{O}+\mathrm{HCO} \rightarrow \mathrm{OH}+\mathrm{CO}$ & $1.0 \cdot 10^{-10}$ & Hampson and Garvin, 1977 \\
\hline 302 & $\mathrm{O}+\mathrm{HNO} \rightarrow \mathrm{OH}+\mathrm{NO}$ & $5.99 \cdot 10^{-11}$ & Tsang and Hampson, 1986 \\
\hline 303 & $\mathrm{O}+\mathrm{O}+\mathrm{M} \rightarrow \mathrm{O}_{2}+\mathrm{M}$ & $9.46 \cdot 10^{-34} \times \mathrm{e}^{480 / \mathrm{T}} \times$ den & Campbell and Gray, 1973 \\
\hline 304 & $\mathrm{O}+\mathrm{O}_{2}+\mathrm{M} \rightarrow \mathrm{O}_{3}+\mathrm{M}$ & $6 \cdot 10^{-34} \times 3 \cdot 10^{-11} \times(\mathrm{T} / 298)^{2.40}$ & Sander et al., 2006 \\
\hline 305 & $\mathrm{O}+\mathrm{O}_{3} \rightarrow \mathrm{O}_{2}+\mathrm{O}_{2}$ & $8.0 \cdot 10^{-12} \times \mathrm{e}^{-2060 / \mathrm{T}}$ & Sander et al., 2006 \\
\hline 306 & $\mathrm{O}^{1} \mathrm{D}+\mathrm{M} \rightarrow \mathrm{O}+\mathrm{M}$ & $1.8 \cdot 10^{-11} \times \mathrm{e}^{110 / \mathrm{T}}$ & Sander et al., 2006 \\
\hline 307 & $\mathrm{O}^{1} \mathrm{D}+\mathrm{O}_{2} \rightarrow \mathrm{O}+\mathrm{O}_{2}$ & $3.2 \cdot 10^{-11} \times \mathrm{e}^{70 / \mathrm{T}}$ & Sander et al., 2006 \\
\hline 308 & $\mathrm{OH}+\mathrm{HCO} \rightarrow \mathrm{H}_{2} \mathrm{O}+\mathrm{CO}$ & $1.7 \cdot 10^{-10}$ & Baulch et al., 1992 \\
\hline 309 & $\mathrm{OH}+\mathrm{HNO} \rightarrow \mathrm{H}_{2} \mathrm{O}+\mathrm{NO}$ & $5 \cdot 10^{-11}$ & Sun et al., 2001 \\
\hline 310 & $\mathrm{OH}+\mathrm{HO}_{2} \rightarrow \mathrm{H}_{2} \mathrm{O}+\mathrm{O}_{2}$ & $4.8 \cdot 10^{-11} \times \mathrm{e}^{250 / \mathrm{T}}$ & Sander et al., 2006 \\
\hline 311 & $\mathrm{OH}+\mathrm{O} \rightarrow \mathrm{H}+\mathrm{O}_{2}$ & $2.2 \cdot 10^{-11} \times \mathrm{e}^{120 / \mathrm{T}}$ & Sander et al., 2006 \\
\hline 312 & $\mathrm{OH}+\mathrm{O}_{3} \rightarrow \mathrm{HO}_{2}+\mathrm{O}_{2}$ & $1.6 \cdot 10^{-12} \times \mathrm{e}^{-940 / \mathrm{T}}$ & Sander et al., 2006 \\
\hline 313 & $\mathrm{OH}+\mathrm{OH} \rightarrow \mathrm{H}_{2} \mathrm{O}+\mathrm{O}$ & $4.2 \cdot 10^{-12} \times \mathrm{e}^{-240 / \mathrm{T}}$ & Sander et al., 2006 \\
\hline 314 & $\mathrm{OH}+\mathrm{OH} \rightarrow \mathrm{H}_{2} \mathrm{O}_{2}$ & $6.9 \cdot 10^{-31} \times 1.5 \cdot 10^{-11} \times(\mathrm{T} / 298)^{0.80}$ & Sander et al., 2006 \\
\hline 315 & $\mathrm{~S}+\mathrm{CO}_{2} \rightarrow \mathrm{SO}+\mathrm{CO}$ & $1.0 \cdot 10^{-20}$ & Yung and Demore, 1982 \\
\hline 316 & $\mathrm{~S}+\mathrm{HCO} \rightarrow \mathrm{HS}+\mathrm{CO}$ & $5.0 \cdot 10^{-11}$ & Kasting, 1990 \\
\hline 317 & $\mathrm{~S}+\mathrm{HO}_{2} \rightarrow \mathrm{HS}+\mathrm{O}_{2}$ & $1.5 \cdot 10^{-11}$ & Kasting, 1990 \\
\hline 318 & $\mathrm{~S}+\mathrm{HO}_{2} \rightarrow \mathrm{SO}+\mathrm{OH}$ & $1.5 \cdot 10^{-11}$ & Kasting, 1990 \\
\hline 319 & $\mathrm{~S}+\mathrm{O}_{2} \rightarrow \mathrm{SO}+\mathrm{O}$ & $2.3 \cdot 10^{-12}$ & Sander et al., 2006 \\
\hline 320 & $\mathrm{~S}+\mathrm{O}_{3} \rightarrow \mathrm{SO}+\mathrm{O}_{2}$ & $1.2 \cdot 10^{-11}$ & Sander et al., 2006 \\
\hline 321 & $\mathrm{~S}+\mathrm{OH} \rightarrow \mathrm{SO}+\mathrm{H}$ & $6.6 \cdot 10^{-11}$ & Sander et al., 2006 \\
\hline 322 & $\mathrm{~S}+\mathrm{S}+\mathrm{M} \rightarrow \mathrm{S}_{2}+\mathrm{M}$ & $1.98 \cdot 10^{-33} \times \mathrm{e}^{-206 / \mathrm{T}} \times$ den & Du et al., 2008 \\
\hline 323 & $\mathrm{~S}+\mathrm{S}_{2}+\mathrm{M} \rightarrow \mathrm{S}_{3}+\mathrm{M}$ & $2.8 \cdot 10^{-32} \times$ den & Kasting, 1990 \\
\hline 324 & $\mathrm{~S}+\mathrm{S}_{3}+\mathrm{M} \rightarrow \mathrm{S}_{4}+\mathrm{M}$ & $2.8 \cdot 10^{-31} \times$ den & Kasting, 1990 \\
\hline 325 & $\mathrm{~S}_{2}+\mathrm{O} \rightarrow \mathrm{S}+\mathrm{SO}$ & $1.1 \cdot 10^{-11}$ & Hills et al., 1987 \\
\hline 326 & $\mathrm{~S}_{2}+\mathrm{S}_{2}+\mathrm{M} \rightarrow \mathrm{S}_{4}+\mathrm{M}$ & $2.8 \cdot 10^{-31} \times$ den & Baulch et al., 1976 \\
\hline
\end{tabular}


TABle 1. (Continued)

\begin{tabular}{|c|c|c|c|}
\hline Rxn. \# & Reaction & Reaction rate constant & Reference \\
\hline 327 & $\mathrm{~S}_{4}+\mathrm{S}_{4}+\mathrm{M} \rightarrow \mathrm{S}_{8} \mathrm{AER}+\mathrm{M}$ & $2.8 \cdot 10^{-31} \times$ den & Kasting, 1990 \\
\hline 328 & $\mathrm{SO}+\mathrm{HCO} \rightarrow \mathrm{HSO}+\mathrm{CO}$ & $5.6 \cdot 10^{-12} \times(\mathrm{T} / 298)^{-0.4}$ & Kasting, 1990 \\
\hline 329 & $\mathrm{SO}+\mathrm{HO}_{2} \rightarrow \mathrm{SO}_{2}+\mathrm{OH}$ & $2.8 \cdot 10^{-11}$ & Kasting, 1990 \\
\hline 330 & $\mathrm{SO}+\mathrm{NO}_{2} \rightarrow \mathrm{SO}_{2}+\mathrm{NO}$ & $1.4 \cdot 10^{-11}$ & Sander et al., 2006 \\
\hline 331 & $\mathrm{SO}+\mathrm{O}+\mathrm{M} \rightarrow \mathrm{SO}_{2}+\mathrm{M}$ & $6.0 \cdot 10^{-31} \times$ den & Sander et al., 2006 \\
\hline 332 & $\mathrm{SO}+\mathrm{O}_{2} \rightarrow \mathrm{O}+\mathrm{SO}_{2}$ & $2.4 \cdot 10^{-13} \times \mathrm{e}^{-2370 / \mathrm{T}}$ & Sander et al., 2006 \\
\hline 333 & $\mathrm{SO}+\mathrm{O}_{3} \rightarrow \mathrm{SO}_{2}+\mathrm{O}_{2}$ & $4.5 \cdot 10^{-12} \times \mathrm{e}^{-1170 / \mathrm{T}}$ & Atkinson et al., 2004 \\
\hline 334 & $\mathrm{SO}+\mathrm{OH} \rightarrow \mathrm{SO}_{2}+\mathrm{H}$ & $8.6 \cdot 10^{-11}$ & Sander et al., 2006 \\
\hline 335 & $\mathrm{SO}+\mathrm{SO} \rightarrow \mathrm{SO}_{2}+\mathrm{S}$ & $3.5 \cdot 10^{-15}$ & Martinez and Herron, 1983 \\
\hline 336 & $\mathrm{SO}_{2}+\mathrm{HO}_{2} \rightarrow \mathrm{SO}_{3}+\mathrm{OH}$ & $8.63 \cdot 10^{-16}$ & Lloyd, 1974 \\
\hline 337 & $\mathrm{SO}_{2}+\mathrm{O}+\mathrm{M} \rightarrow \mathrm{SO}_{3}+\mathrm{M}$ & $\begin{array}{l}k_{0}=1.3 \cdot 10^{-33} \times(\mathrm{T} / 298)^{-3.6} \\
k_{\infty}=1.5 \cdot 10^{-11}\end{array}$ & Sander et al., 2006 \\
\hline 338 & $\mathrm{SO}_{2}+\mathrm{OH}+\mathrm{M} \rightarrow \mathrm{HSO}_{3}+\mathrm{M}$ & $\begin{array}{l}k_{0}=3 \cdot 10^{-31} \times(\mathrm{T} / 298)^{3.3} \\
k_{\infty}=1.5 \cdot 10^{-12}\end{array}$ & Sander et al., 2006 \\
\hline 339 & $\mathrm{SO}_{2}^{1}+\mathrm{M} \rightarrow \mathrm{SO}_{2}+\mathrm{M}$ & $1.0 \cdot 10^{-11}$ & Turco et al., 1982 \\
\hline 340 & $\mathrm{SO}_{2}^{1}+\mathrm{M} \rightarrow \mathrm{SO}_{2}^{3}+\mathrm{M}$ & $1.0 \cdot 10^{-12}$ & Turco et al., 1982 \\
\hline 341 & $\mathrm{SO}_{2}{ }^{1}+\mathrm{O}_{2} \rightarrow \mathrm{SO}_{3}+\mathrm{O}$ & $1.0 \cdot 10^{-16}$ & Turco et al., 1982 \\
\hline 342 & $\mathrm{SO}_{2}^{1}+\mathrm{SO}_{2} \rightarrow \mathrm{SO}_{3}+\mathrm{SO}$ & $4.0 \cdot 10^{-12}$ & Turco et al., 1982 \\
\hline 343 & $\mathrm{SO}_{2}^{3}+\mathrm{M} \rightarrow \mathrm{SO}_{2}+\mathrm{M}$ & $1.5 \cdot 10^{-13}$ & Turco et al., 1982 \\
\hline 344 & $\mathrm{SO}_{2}^{3}+\mathrm{SO}_{2} \rightarrow \mathrm{SO}_{3}+\mathrm{SO}$ & $7.0 \cdot 10^{-14}$ & Turco et al., 1982 \\
\hline 345 & $\mathrm{SO}_{3}+\mathrm{H}_{2} \mathrm{O} \rightarrow \mathrm{H}_{2} \mathrm{SO}_{4}$ & $1.2 \cdot 10^{-15}$ & Sander et al., 2006 \\
\hline 346 & $\mathrm{SO}_{3}+\mathrm{SO} \rightarrow \mathrm{SO}_{2}+\mathrm{SO}_{2}$ & $2.0 \cdot 10^{-15}$ & Chung et al., 1975 \\
\hline 347 & $\mathrm{SO}_{2}^{1}+h \nu \rightarrow \mathrm{SO}_{2}+h \nu$ & $2.2 \cdot 10^{+4}$ & Turco et al., 1982 \\
\hline 348 & $\mathrm{SO}_{2}^{1}+h \nu \rightarrow \mathrm{SO}_{2}^{3}+h \nu$ & $1.5 \cdot 10^{+3}$ & Turco et al., 1982 \\
\hline 349 & $\mathrm{SO}_{2}^{3}+h \nu \rightarrow \mathrm{SO}_{2}+h \nu$ & $1.13 \cdot 10^{+3}$ & Turco et al., 1982 \\
\hline 350 & $\mathrm{O}_{2}+h v \rightarrow \mathrm{O}+\mathrm{O}^{1} \mathrm{D}$ & $1.51 \cdot 10^{+02}$ & \\
\hline 351 & $\mathrm{O}_{2}+h v \rightarrow \mathrm{O}+\mathrm{O}$ & $2.90 \cdot 10^{+00}$ & \\
\hline 352 & $\mathrm{H}_{2} \mathrm{O}+h v \rightarrow \mathrm{H}+\mathrm{OH}$ & $1.65 \cdot 10^{-01}$ & \\
\hline 353 & $\mathrm{O}_{3}+h v \rightarrow \mathrm{O}_{2}+\mathrm{O}^{1} \mathrm{D}$ & $6.44 \cdot 10^{-04}$ & \\
\hline 354 & $\mathrm{O}_{3}+h v \rightarrow \mathrm{O}_{2}+\mathrm{O}$ & $1.64 \cdot 10^{-04}$ & \\
\hline 355 & $\mathrm{H}_{2} \mathrm{O}_{2}+h v \rightarrow \mathrm{OH}+\mathrm{OH}$ & $2.79 \cdot 10^{-14}$ & \\
\hline 356 & $\mathrm{CO}_{2}+h v \rightarrow \mathrm{CO}+\mathrm{O}$ & $2.50 \cdot 10^{+01}$ & \\
\hline 357 & $\mathrm{H}_{2} \mathrm{CO}+h v \rightarrow \mathrm{H}_{2}+\mathrm{CO}$ & $7.71 \cdot 10^{-01}$ & \\
\hline 358 & $\mathrm{H}_{2} \mathrm{CO}+h v \rightarrow \mathrm{HCO}+\mathrm{H}$ & $9.33 \cdot 10^{-01}$ & \\
\hline 359 & $\mathrm{CO}_{2}+h v \rightarrow \mathrm{CO}+\mathrm{O}^{1} \mathrm{D}$ & $2.73 \cdot 10^{+03}$ & \\
\hline 360 & $\mathrm{HO}_{2}+h v \rightarrow \mathrm{OH}+\mathrm{O}$ & $0.00 \cdot 10^{+00}$ & \\
\hline 361 & $\mathrm{CH}_{4}+h \nu \rightarrow \mathrm{CH}_{2}^{1}+\mathrm{H}_{2}$ & $1.75 \cdot 10^{+00}$ & \\
\hline 362 & $\mathrm{C}_{2} \mathrm{H}_{6}+h \nu \rightarrow \mathrm{CH}_{2}^{3}+\mathrm{CH}_{2}^{3}+\mathrm{H}_{2}$ & 0.00 & \\
\hline 363 & $\mathrm{C}_{2} \mathrm{H}_{6}+h \nu \rightarrow \mathrm{CH}_{4}+\mathrm{CH}_{2}^{1}$ & $1.48 \cdot 10^{-05}$ & \\
\hline 364 & $\mathrm{HNO}_{2}+h v \rightarrow \mathrm{NO}+\mathrm{OH}$ & $8.68 \cdot 10^{-22}$ & \\
\hline 365 & $\mathrm{HNO}_{3}+h v \rightarrow \mathrm{NO}_{2}+\mathrm{OH}$ & $2.74 \cdot 10^{-28}$ & \\
\hline 366 & $\mathrm{NO}+h v \rightarrow \mathrm{N}+\mathrm{O}$ & $2.04 \cdot 10^{-10}$ & \\
\hline 367 & $\mathrm{NO}_{2}+h v \rightarrow \mathrm{NO}+\mathrm{O}$ & $4.40 \cdot 10^{-14}$ & \\
\hline 368 & $\mathrm{CH}_{3}+h \nu \rightarrow \mathrm{CH}_{2}^{1}+\mathrm{H}$ & $6.67 \cdot 10^{-04}$ & \\
\hline 369 & $\mathrm{SO}+h v \rightarrow \mathrm{S}+\mathrm{O}$ & $0.00 \cdot 10^{+00}$ & \\
\hline 370 & $\mathrm{SO}_{2}+h v \rightarrow \mathrm{SO}+\mathrm{O}$ & $1.37 \cdot 10^{-10}$ & \\
\hline 371 & $\mathrm{H}_{2} \mathrm{~S}+h v \rightarrow \mathrm{HS}+\mathrm{H}$ & $1.00 \cdot 10^{-23}$ & \\
\hline 372 & $\mathrm{SO}_{2}+h \nu \rightarrow \mathrm{SO}_{2}^{1}$ & $1.52 \cdot 10^{-09}$ & \\
\hline 373 & $\mathrm{SO}_{2}+h \nu \rightarrow \mathrm{SO}_{2}^{3}$ & $8.14 \cdot 10^{-13}$ & \\
\hline 374 & $\mathrm{~S}_{2}+h v \rightarrow \mathrm{S}+\mathrm{S}$ & $5.94 \cdot 10^{-42}$ & \\
\hline 375 & $\mathrm{~S}_{2}+h v \rightarrow \mathrm{S}_{2}$ & $0.00 \cdot 10^{+00}$ & \\
\hline 376 & $\mathrm{H}_{2} \mathrm{SO}_{4}+h v \rightarrow \mathrm{SO}_{2}+\mathrm{OH}+\mathrm{OH}$ & $1.66 \cdot 10^{-13}$ & \\
\hline 377 & $\mathrm{SO}_{3}+h v \rightarrow \mathrm{SO}_{2}+\mathrm{O}$ & $0.00 \cdot 10^{+00}$ & \\
\hline 378 & $\mathrm{SO}_{2}^{1}+h \nu \rightarrow \mathrm{SO}_{2}^{3}+h \nu$ & $9.70 \cdot 10^{-11}$ & \\
\hline 379 & $\mathrm{SO}_{2}{ }^{1}+h \nu \rightarrow \mathrm{SO}_{2}+h \nu$ & $1.42 \cdot 10^{-09}$ & \\
\hline 380 & $\mathrm{SO}_{2}^{3}+h \nu \rightarrow \mathrm{SO}_{2}+h \nu$ & $9.78 \cdot 10^{-11}$ & \\
\hline 381 & $\mathrm{HSO}+h v \rightarrow \mathrm{HS}+\mathrm{O}$ & $7.19 \cdot 10^{-17}$ & \\
\hline 382 & $\mathrm{~S}_{4}+h v \rightarrow \mathrm{S}_{2}+\mathrm{S}_{2}$ & $0.00 \cdot 10^{+00}$ & \\
\hline 383 & $\mathrm{~S}_{3}+h v \rightarrow \mathrm{S}_{2}+\mathrm{S}$ & $4.22 \cdot 10^{-72}$ & \\
\hline 384 & $\mathrm{NH}_{3}+h v \rightarrow \mathrm{NH}_{2}+\mathrm{H}$ & $6.00 \cdot 10^{-34}$ & \\
\hline 385 & $\mathrm{~N}_{2} \mathrm{H}_{4}+h v \rightarrow \mathrm{N}_{2} \mathrm{H}_{3}+\mathrm{H}$ & $9.75 \cdot 10^{-93}$ & \\
\hline 386 & $\mathrm{NH}+h v \rightarrow \mathrm{N}+\mathrm{H}$ & $3.99 \cdot 10^{-35}$ & \\
\hline
\end{tabular}


TAble 1. (Continued)

\begin{tabular}{|c|c|c|c|}
\hline Rxn. \# & Reaction & Reaction rate constant & Reference \\
\hline 387 & $\mathrm{NH}_{2}+h v \rightarrow \mathrm{NH}+\mathrm{H}$ & $7.49 \cdot 10^{-37}$ & \\
\hline 388 & $\mathrm{NH}_{2}+h \nu \rightarrow \mathrm{NH}_{2}^{*}$ & $3.99 \cdot 10^{-35}$ & \\
\hline 389 & $\mathrm{NH}_{2}^{*}+h \nu \rightarrow \mathrm{NH}_{2}+h \nu$ & $3.99 \cdot 10^{-35}$ & \\
\hline 390 & $\mathrm{C}_{2} \mathrm{H}_{2}+h v \rightarrow \mathrm{C}_{2} \mathrm{H}+\mathrm{H}$ & $5.51 \cdot 10^{-07}$ & \\
\hline 391 & $\mathrm{C}_{2} \mathrm{H}_{2}+h v \rightarrow \mathrm{C}_{2}+\mathrm{H}_{2}$ & $4.09 \cdot 10^{-07}$ & \\
\hline 392 & $\mathrm{C}_{2} \mathrm{H}_{4}+h v \rightarrow \mathrm{C}_{2} \mathrm{H}_{2}+\mathrm{H}_{2}$ & $5.51 \cdot 10^{-07}$ & \\
\hline 393 & $\mathrm{C}_{3} \mathrm{H}_{8}+h v \rightarrow \mathrm{C}_{3} \mathrm{H}_{6}+\mathrm{H}_{2}$ & $1.45 \cdot 10^{-12}$ & \\
\hline 394 & $\mathrm{C}_{3} \mathrm{H}_{8}+h \nu \rightarrow \mathrm{C}_{2} \mathrm{H}_{6}+\mathrm{CH}_{2}{ }^{1}$ & $2.49 \cdot 10^{-13}$ & \\
\hline 395 & $\mathrm{C}_{3} \mathrm{H}_{8}+h v \rightarrow \mathrm{C}_{2} \mathrm{H}_{4}+\mathrm{CH}_{4}$ & $1.08 \cdot 10^{-12}$ & \\
\hline 396 & $\mathrm{C}_{3} \mathrm{H}_{8}+h v \rightarrow \mathrm{C}_{2} \mathrm{H}_{5}+\mathrm{CH}_{3}$ & $5.88 \cdot 10^{-13}$ & \\
\hline 397 & $\mathrm{C}_{2} \mathrm{H}_{6}+h v \rightarrow \mathrm{C}_{2} \mathrm{H}_{2}+\mathrm{H}_{2}+\mathrm{H}_{2}$ & $1.80 \cdot 10^{-05}$ & \\
\hline 398 & $\mathrm{C}_{2} \mathrm{H}_{6}+h v \rightarrow \mathrm{C}_{2} \mathrm{H}_{4}+\mathrm{H}+\mathrm{H}$ & $1.93 \cdot 10^{-05}$ & \\
\hline 399 & $\mathrm{C}_{2} \mathrm{H}_{6}+h v \rightarrow \mathrm{C}_{2} \mathrm{H}_{4}+\mathrm{H}_{2}$ & $5.29 \cdot 10^{-07}$ & \\
\hline 400 & $\mathrm{C}_{2} \mathrm{H}_{6}+h v \rightarrow \mathrm{CH}_{3}+\mathrm{CH}_{3}$ & $4.79 \cdot 10^{-06}$ & \\
\hline 401 & $\mathrm{C}_{2} \mathrm{H}_{4}+h v \rightarrow \mathrm{C}_{2} \mathrm{H}_{2}+\mathrm{H}+\mathrm{H}$ & $5.29 \cdot 10^{-07}$ & \\
\hline 402 & $\mathrm{C}_{3} \mathrm{H}_{6}+h v \rightarrow \mathrm{C}_{2} \mathrm{H}_{2}+\mathrm{CH}_{3}+\mathrm{H}$ & $5.26 \cdot 10^{-16}$ & \\
\hline 403 & $\mathrm{CH}_{4}+h \nu \rightarrow \mathrm{CH}_{2}^{3}+\mathrm{H}+\mathrm{H}$ & $1.42 \cdot 10^{+00}$ & \\
\hline 404 & $\mathrm{CH}_{4}+h v \rightarrow \mathrm{CH}_{3}+\mathrm{H}$ & $2.91 \cdot 10^{+00}$ & \\
\hline 405 & $\mathrm{CH}+h v \rightarrow \mathrm{C}+\mathrm{H}$ & $9.52 \cdot 10^{-06}$ & \\
\hline 406 & $\mathrm{CH}_{2} \mathrm{CO}+h \nu \rightarrow \mathrm{CH}_{2}{ }^{3}+\mathrm{CO}$ & $8.21 \cdot 10^{-10}$ & \\
\hline 407 & $\mathrm{CH}_{3} \mathrm{CHO}+h v \rightarrow \mathrm{CH}_{3}+\mathrm{HCO}$ & $1.14 \cdot 10^{-08}$ & \\
\hline 408 & & $114 \cdot 10^{-08}$ & \\
\hline & $\mathrm{CH}_{3} \mathrm{CHU}+h v \rightarrow \mathrm{CH}_{4}+\mathrm{CO}$ & & \\
\hline 409 & $\mathrm{C}_{2} \mathrm{H}_{5} \mathrm{CHO}+h v \rightarrow \mathrm{C}_{2} \mathrm{H}_{5}+\mathrm{HCO}$ & $6.42 \cdot 10^{-07}$ & \\
\hline 410 & $\mathrm{C}_{3} \mathrm{H}_{3}+h v \rightarrow \mathrm{C}_{3} \mathrm{H}_{2}+\mathrm{H}$ & $6.88 \cdot 10^{-07}$ & \\
\hline 411 & $\mathrm{CH}_{3} \mathrm{C}_{2} \mathrm{H}+h v \rightarrow \mathrm{C}_{3} \mathrm{H}_{3}+\mathrm{H}$ & $6.42 \cdot 10^{-07}$ & \\
\hline 412 & $\mathrm{CH}_{3} \mathrm{C}_{2} \mathrm{H}+h v \rightarrow \mathrm{C}_{3} \mathrm{H}_{2}+\mathrm{H}_{2}$ & $2.41 \cdot 10^{-07}$ & \\
\hline 413 & $\mathrm{CH}_{3} \mathrm{C}_{2} \mathrm{H}+h v \rightarrow \mathrm{CH}_{3}+\mathrm{C}_{2} \mathrm{H}$ & $3.21 \cdot 10^{-08}$ & \\
\hline 414 & $\mathrm{CH}_{2} \mathrm{CCH}_{2}+h v \rightarrow \mathrm{C}_{3} \mathrm{H}_{3}+\mathrm{H}$ & $6.49 \cdot 10^{-13}$ & \\
\hline 415 & $\mathrm{CH}_{2} \mathrm{CCH}_{2}+h v \rightarrow \mathrm{C}_{3} \mathrm{H}_{2}+\mathrm{H}_{2}$ & $2.43 \cdot 10^{-13}$ & \\
\hline 416 & $\mathrm{CH}_{2} \mathrm{CCH}_{2}+h \nu \rightarrow \mathrm{C}_{2} \mathrm{H}_{2}+\mathrm{CH}_{2}^{3}$ & $9.73 \cdot 10^{-14}$ & \\
\hline 417 & $\mathrm{C}_{3} \mathrm{H}_{6}+h v \rightarrow \mathrm{CH}_{2} \mathrm{CCH}_{2}+\mathrm{H}_{2}$ & $8.81 \cdot 10^{-16}$ & \\
\hline 418 & $\mathrm{C}_{3} \mathrm{H}_{6}+h \nu \rightarrow \mathrm{C}_{2} \mathrm{H}_{4}+\mathrm{CH}_{2}^{3}$ & $3.09 \cdot 10^{-17}$ & \\
\hline 419 & $\mathrm{C}_{3} \mathrm{H}_{6}+h v \rightarrow \mathrm{C}_{2} \mathrm{H}+\mathrm{CH}_{4}+\mathrm{H}$ & $1.43 \cdot 10^{-10}$ & \\
\hline 420 & $\mathrm{OCS}+h v \rightarrow \mathrm{CO}+\mathrm{S}$ & $2.67 \cdot 10^{-36}$ & \\
\hline 421 & $\mathrm{CS}_{2}+h v \rightarrow \mathrm{CS}+\mathrm{S}$ & $5.40 \cdot 10^{-47}$ & \\
\hline 422 & $\mathrm{CH}_{3} \mathrm{SH}+h v \rightarrow \mathrm{H}+\mathrm{CH}_{3} \mathrm{~S}$ & $1.48 \cdot 10^{-30}$ & \\
\hline 423 & $\mathrm{CH}_{3} \mathrm{SH}+h v \rightarrow \mathrm{HS}+\mathrm{CH}_{3}$ & $1.11 \cdot 10^{-31}$ & \\
\hline 424 & $\mathrm{C}_{2} \mathrm{H}_{6} \mathrm{~S}+h v \rightarrow \mathrm{CH}_{3} \mathrm{~S}+\mathrm{CH}_{3}$ & $4.01 \cdot 10^{-93}$ & \\
\hline 425 & $\mathrm{C}_{2} \mathrm{H}_{6} \mathrm{~S}_{2}+h v \rightarrow \mathrm{CH}_{3} \mathrm{~S}+\mathrm{CH}_{3} \mathrm{~S}$ & $1.65 \cdot 10^{-34}$ & \\
\hline 426 & $\mathrm{CS}_{2}+h \nu \rightarrow \mathrm{CS}_{2}^{*}$ & $6.57 \cdot 10^{-48}$ & \\
\hline
\end{tabular}

For photolysis reactions (bottom of table), the "Reaction rate constant" column shows the reaction rate (not the rate constant) at the top of the atmosphere during our "standard" simulation, the modern-day fluxes of $\mathrm{CH}_{4}, \mathrm{H}_{2} \mathrm{~S}$, and the $\mathrm{S}_{\text {org }}$ species on a planet orbiting the Sun. For more on how to calculate reaction rates, see Sander et al. (2006).

$\mathrm{O}_{2} / \mathrm{O}_{3}$ should not be taken as evidence that life does not exist on a planet's surface.

Furthermore, some planets and biospheres will not exhibit the more general feature of photochemical disequilibrium previously proposed as a universal biosignature (Lederberg, 1965; Lovelock, 1965; Des Marais et al., 2002). Unlike Earth's modern-day ecosystem, global anoxic ecosystems may drive an atmosphere toward equilibrium. For example, in the anoxic Archean biospheres considered by Kharecha et al. (2005), methanogens and acetogens combine $\mathrm{H}_{2}$ and $\mathrm{CO}$ with $\mathrm{CO}_{2}$ and $\mathrm{H}_{2} \mathrm{O}$ to produce $\mathrm{CH}_{4}$. They can make a metabolic living by doing this because $\mathrm{CH}_{4}$ has a lower Gibbs free energy and hence is thermodynamically stable in such a system. The biogenic gases released from such a biosphere result from a drive toward equilibrium, not disequilibrium. Because cases like these could complicate interpretation, it is important to identify additional biosignature gases that might be signs of anoxic biospheres. In this paper, we test the ability of various gases with carbon-sulfur bonds to act as remotely detectable biosignatures for anoxic, inhabited surface environments.

The biosignature potential of S-bearing gases was reviewed by Pilcher (2003), who focused on gases with bonds between methyl groups $\left(-\mathrm{CH}_{3}\right)$ and sulfur: methanethiol $\left(\mathrm{CH}_{3} \mathrm{SH}\right.$, also known as methyl mercaptan), dimethyl sulfide $\left(\mathrm{CH}_{3} \mathrm{SCH}_{3}\right.$ or DMS), and dimethyl disulfide $\left(\mathrm{CH}_{3} \mathrm{~S}_{2} \mathrm{CH}_{3}\right.$ or DMDS). More recently, Vance et al. (2011) suggested that $\mathrm{CH}_{3} \mathrm{SH}$ could be used as an in situ signature for life on Mars. On modern Earth, the production of these species is dominated by biota, but they are rapidly destroyed by photolysis and by reaction with hydroxyl $(\mathrm{OH})$ radicals (Kettle et al., 2001), and do not build up to concentrations detectable across interstellar distances. In this work, we consider these gases, along with carbon disulfide $\left(\mathrm{CS}_{2}\right)$ and carbonyl sulfide (OCS, sometimes abbreviated in other work as COS), two 
other biogenic gases that contain carbon-sulfur bonds. These two species-particularly OCS-also have volcanic and photochemical sources, but they are far smaller than biological fluxes. We henceforth use the term " $\mathrm{S}_{\text {org }}$ " as shorthand to refer to the entire suite of biologically produced species with carbon-sulfur bonds (DMS, DMDS, $\mathrm{CH}_{3} \mathrm{SH}, \mathrm{CS}_{2}$, and OCS). Although hydrogen sulfide $\left(\mathrm{H}_{2} \mathrm{~S}\right)$ is another S-bearing gas produced by biota, large quantities of this species enter the atmosphere via volcanism. Thus, we do not consider it here as a biosignature. However, we do consider the possibility that volcanic $\mathrm{H}_{2} \mathrm{~S}$ could act as a "false positive" for biogenic $\mathrm{S}_{\text {org }}$, as this abiotic $\mathrm{H}_{2} \mathrm{~S}$ could react in the atmosphere to form $\mathrm{S}_{\text {org }}$ species. Other work has explored the spectral signatures of sulfur dioxide $\left(\mathrm{SO}_{2}\right)$ and $\mathrm{H}_{2} \mathrm{~S}$ in detail (Kaltenegger and Sasselov, 2010), so we limit our discussion to their potential to be false positives for biological $S_{\text {org }}$ production. No study to date has predicted the concentrations of all the $S_{\text {org }}$ species in an anoxic atmosphere, nor has any study predicted the spectral features associated with these gases. We used a photochemical model to calculate vertical profiles of these gases for a variety of astronomical and biological contexts, and used a radiative transfer model to predict the spectral features consistent with those profiles.

\section{Methods}

\subsection{Photochemical code}

We modified the one-dimensional (altitude), low- $\mathrm{O}_{2}$ photochemical code originally developed by Kasting et al. (1979) to study the anoxic early Earth. The numerics of this model are described by Kasting and Ackerman (1985), and the chemistry was most recently modified by Pavlov et al. (2001). We have updated this code, adding seven long-lived chemical species that have lifetimes longer than the time scale for vertical mixing: $\mathrm{CH}_{3} \mathrm{SH}$, DMS, DMDS, OCS, $\mathrm{CS}_{2}$, methylthiol $\left(\mathrm{CH}_{3} \mathrm{~S}\right)$, and carbon monosulfide (CS). We also added three short-lived species, which are solved in photochemical equilibrium without considering vertical transport: excited-state $\mathrm{CS}_{2}, \mathrm{OCS}_{2}$, and HCS. These 10 species were incorporated into the chemical scheme by adding 73 chemical reactions. The current model contains 83 chemical species, 46 of which are long lived, connected by 433 chemical reactions. Additionally, many of the 360 reactions from prior work have updated reaction rate constants. A complete list of model reactions, reaction rate constants, and references can be found in Table 1 .

The model grid is composed of 100 plane-parallel layers that are each $1 \mathrm{~km}$ thick in altitude. We did not perform climate calculations for this work; instead, we assumed a temperature profile for an aerosol-free, ozone-free atmosphere. This profile had a surface temperature of $278 \mathrm{~K}$ that decreased to $180 \mathrm{~K}$ at the tropopause and was isothermal through the stratosphere. The relatively low surface temperature was picked for consistency with previous Archean photochemistry and climate models (Haqq-Misra et al., 2008), and the isothermal stratosphere is consistent with the model's lack of $\mathrm{O}_{3}$. The code calculates the mixing ratios of each species in each layer by solving the coupled mass-continuity/flux equations with the reverse Euler method (appropriate for stiff systems) and a variable time-stepping algorithm. For further details on the photochemical code, see Pavlov et al. (2001) and references therein.
Unless otherwise stated, all model runs were for a 1-bar, $\mathrm{N}_{2}$-dominated atmosphere with $3 \% \mathrm{CO}_{2} \quad(30,000 \mathrm{ppmv}$, or $\sim 100$ times the present level of $\mathrm{CO}_{2}$ in Earth's atmosphere) and $\mathrm{CH}_{4} / \mathrm{CO}_{2}$ ratios $<0.1$. These boundary conditions prevent formation of a significant organic haze (Pavlov et al., 2001; Trainer et al., 2006; Domagal-Goldman et al., 2008). These concentrations and the model's other chemical boundary conditions are by no means unique; however, they were chosen for consistency with a methanogen-acetogen ecosystem (Kharecha et al., 2005). The modeling of haze-free atmospheres is, from a photochemical standpoint, conservative. Including haze in the model would shield the gases we are studying from UV radiation and thereby increase their mixing ratios.

\subsection{Boundary conditions}

At the top of the atmosphere we allowed $\mathrm{H}$ and $\mathrm{H}_{2}$ to escape at the diffusion-limited rate (Walker, 1977). We also applied a constant downward flux of $\mathrm{CO}$ and $\mathrm{O}$ at the top of our model atmosphere. This accounts for $\mathrm{CO}$ and $\mathrm{O}$ that is produced from $\mathrm{CO}_{2}$ photolysis above the top layer of our atmosphere and subsequently flows downward into the model grid. For all other species, we used a zero-flux boundary condition at the top of the atmosphere (i.e., no escape).

At the bottom of the atmosphere, we used constant deposition velocities (to account for reactions with surface rocks and for dissolution in the ocean) for all species except the $\mathrm{S}_{\text {org }}$ species, $\mathrm{CH}_{4}$, and $\mathrm{NH}_{3}$. In addition to constant deposition velocities, $\mathrm{H}_{2} \mathrm{~S}, \mathrm{SO}_{2}$, and $\mathrm{H}_{2}$ had volcanic fluxes of $1 \times 10^{9}$ molecules $/ \mathrm{cm}^{2} / \mathrm{s}, 1 \times 10^{10}$ molecules $/ \mathrm{cm}^{2} / \mathrm{s}$, and $3 \times 10^{10}$ molecules $/ \mathrm{cm}^{2} / \mathrm{s}$, respectively, consistent with past models of Archean Earth (Zahnle et al., 2006) that assume volcanism rates about 3 times modern-day values. These fluxes were distributed throughout the troposphere to simulate volcanism. $\mathrm{CH}_{4}$ was modeled with a constant flux of $200 \mathrm{Tg} \mathrm{C} /$ year $\left(7 \times 10^{10}\right.$ molecules $\left./ \mathrm{cm}^{2} / \mathrm{s}\right)$ into the bottom layer of the atmosphere, in line with estimates of modernday non-anthropogenic fluxes on Earth (Intergovernmental Panel on Climate Change, 2007). [The total $\mathrm{CH}_{4}$ flux today is about 2 times higher; see the Intergovernmental Panel on Climate Change (2007)]. Despite this modern-day flux, the concentrations of $\mathrm{CH}_{4}$ in our models were much higher than they are today because the lack of atmospheric $\mathrm{O}_{2}$ allowed $\mathrm{CH}_{4}$ to accumulate. We imposed a constant mixing ratio of $10^{-10}$ for $\mathrm{NH}_{3}$. The corresponding surface flux needed to maintain this mixing ratio was $12.4 \mathrm{Tg} \mathrm{N} /$ year, slightly larger than the present-day non-anthropogenic $\mathrm{NH}_{3}$ flux, $10.5 \mathrm{Tg} \mathrm{N} /$ year (Intergovernmental Panel on Climate Change, 2007). All photochemical boundary conditions are listed in Table 2.

We parameterized the biological production of $\mathrm{S}_{\text {org }}$. The modern-day $S_{\text {org }}$ fluxes, predominantly biological in source, are as follows (in units of molecules $/ \mathrm{cm}^{2} / \mathrm{s}$ ): 0 for DMDS, $4.2 \times 10^{9}$ for DMS, 0 for $\mathrm{CH}_{3} \mathrm{~S}, 8.3 \times 10^{8}$ for $\mathrm{CH}_{3} \mathrm{SH}, 1.4 \times 10^{7}$ for $\mathrm{CS}_{2}, 1.4 \times 10^{7}$ for OCS, and 0 for CS (Kettle et al., 2001). We will use "MDF" as a unit to represent these modern-day fluxes in the rest of this paper, such that $1 \mathrm{MDF} \mathrm{S}_{\text {org }}$ is equivalent to an atmosphere that receives all $S_{\text {org }}$ species at the above fluxes. DMDS, $\mathrm{CH}_{3} \mathrm{~S}$, and CS have zero direct biological production but are produced photochemically from 
Table 2. A List of Species in Our Photochemical Code Along With the Lower Boundary Condition Type AND VAlues, the LATTER Given in CGS Units: CM/s for Deposition Velocity $\left(V_{\text {dep }}\right)$, Dimensionless Mixing Ratio by Volume for Fixed Concentration $\left(f_{0}\right)$, AND MOLECUles $/ \mathrm{CM}^{2} / \mathrm{s}$ FOR Flux (flux)

\begin{tabular}{|c|c|c|}
\hline Species & Lower boundary type & $V_{d e p} / f_{0} / f l u x$ \\
\hline $\mathrm{O}$ & constant deposition velocity & 1 \\
\hline $\mathrm{O}_{2}$ & constant deposition velocity & $1 \cdot 10^{-04}$ \\
\hline $\mathrm{H}_{2} \mathrm{O}$ & constant deposition velocity & 0 \\
\hline $\mathrm{H}$ & constant deposition velocity & 1 \\
\hline $\mathrm{OH}$ & constant deposition velocity & 1 \\
\hline $\mathrm{HO}_{2}$ & constant deposition velocity & 1 \\
\hline $\mathrm{H}_{2} \mathrm{O}_{2}$ & constant deposition velocity & $2 \cdot 10^{-01}$ \\
\hline $\mathrm{H}_{2}$ & constant deposition velocity* & $2.4 \cdot 10^{-04}$ \\
\hline $\mathrm{CO}$ & constant deposition velocity & $1.2 \cdot 10^{-04}$ \\
\hline $\mathrm{HCO}$ & constant deposition velocity & 1 \\
\hline $\mathrm{H}_{2} \mathrm{CO}$ & constant deposition velocity & $2 \cdot 10^{-01}$ \\
\hline $\mathrm{CH}_{4}$ & constant flux & $7 \cdot 10^{+10}$ \\
\hline $\mathrm{CH}_{3}$ & constant deposition velocity & 1 \\
\hline $\mathrm{C}_{2} \mathrm{H}_{6}$ & constant deposition velocity & 0 \\
\hline $\mathrm{NO}$ & constant deposition velocity & $3 \cdot 10^{-04}$ \\
\hline $\mathrm{NO}_{2}$ & constant deposition velocity & $3 \cdot 10^{-03}$ \\
\hline $\mathrm{HNO}$ & constant deposition velocity & 1 \\
\hline $\mathrm{H}_{2} \mathrm{~S}$ & constant deposition velocity* & $2 \cdot 10^{-02}$ \\
\hline $\mathrm{HS}$ & constant deposition velocity & 1 \\
\hline S & constant deposition velocity & 1 \\
\hline $\mathrm{SO}$ & constant deposition velocity & $3 \cdot 10^{-04}$ \\
\hline $\mathrm{SO}_{2}$ & constant deposition velocity* & 1 \\
\hline $\mathrm{H}_{2} \mathrm{SO}_{4}$ & constant deposition velocity & 1 \\
\hline $\mathrm{HSO}$ & constant deposition velocity & 1 \\
\hline $\mathrm{S}_{2}$ & constant deposition velocity & 0 \\
\hline $\mathrm{NH}_{3}$ & constant mixing ratio & $1 \cdot 10^{-10}$ \\
\hline $\mathrm{NH}_{2}$ & constant deposition velocity & 1 \\
\hline $\mathrm{N}_{2} \mathrm{H}_{3}$ & constant deposition velocity & 1 \\
\hline $\mathrm{N}_{2} \mathrm{H}_{4}$ & constant deposition velocity & $2 \cdot 10^{-01}$ \\
\hline $\mathrm{CH}_{2}^{3}$ & constant deposition velocity & 0 \\
\hline $\mathrm{C}_{2} \mathrm{H}_{5}^{2}$ & constant deposition velocity & 0 \\
\hline $\mathrm{C}_{2} \mathrm{H}_{2}$ & constant deposition velocity & 0 \\
\hline $\mathrm{C}_{2} \mathrm{H}_{4}$ & constant deposition velocity & 0 \\
\hline $\mathrm{C}_{3} \mathrm{H}_{8}$ & constant deposition velocity & 0 \\
\hline $\mathrm{C}_{2} \mathrm{H}_{3}$ & constant deposition velocity & 0 \\
\hline $\mathrm{C}_{3} \mathrm{H}_{6}$ & constant deposition velocity & 0 \\
\hline $\mathrm{C}_{3} \mathrm{H}_{2}$ & constant deposition velocity & 0 \\
\hline $\mathrm{CH}_{2} \mathrm{CCH}_{2}$ & constant deposition velocity & 0 \\
\hline $\mathrm{CH}_{3} \mathrm{C}_{2} \mathrm{H}$ & constant deposition velocity & 0 \\
\hline $\mathrm{C}_{2} \mathrm{H}_{6} \mathrm{~S}_{2}$ (DMDS) & constant flux & 0 \\
\hline $\mathrm{C}_{2} \mathrm{H}_{6} \mathrm{~S}(\mathrm{DMS})$ & constant flux & $4.20 \cdot 10^{+09}$ \\
\hline $\mathrm{CH}_{3} \mathrm{~S}$ & constant deposition velocity & $1 \cdot 10^{-02}$ \\
\hline $\mathrm{CH}_{3} \mathrm{SH}$ & constant flux & $8.3 \cdot 10^{+08}$ \\
\hline $\mathrm{CS}_{2}$ & constant flux & $1.4 \cdot 10^{+07}$ \\
\hline OCS & constant flux & $1.4 \cdot 10^{+07}$ \\
\hline CS & constant deposition velocity & $1 \cdot 10^{-04}$ \\
\hline
\end{tabular}

*In addition to a constant deposition velocity, we also use a volcanic flux for these gases. Specifically, we used volcanic fluxes of $3 \cdot 10^{10}$ molecules $/ \mathrm{cm}^{2} / \mathrm{s}$ of $\mathrm{H}_{2}, 1 \cdot 10^{10}$ molecules $/ \mathrm{cm}^{2} / \mathrm{s}$ of $\mathrm{SO}_{2}$, and $1 \cdot 10^{9}$ molecules $/ \mathrm{cm}^{2} / \mathrm{s}$ of $\mathrm{H}_{2} \mathrm{~S}$

other $S_{\text {org }}$ species and are needed to ensure a comprehensive modeling of $S_{\text {org }}$ chemistry. To determine the effect of $S_{\text {org }}$ fluxes on $S_{\text {org }}$ mixing ratios and ultimately on disc-averaged planetary spectra, we parameterized $S_{\text {org }}$ flux rates by holding the ratios of these fluxes constant and multiplying each flux by a common factor.
Most $S_{\text {org }}$ species are produced via methylation of (addition of methyl groups to) $\mathrm{CH}_{3} \mathrm{SH}$ or dehydrogenation of (removal of $\mathrm{H}$ atoms from) $\mathrm{CH}_{3} \mathrm{SH}$, or both. The main modern-day global source of $\mathrm{CH}_{3} \mathrm{SH}$ is the degradation of methionine, an amino acid that contains a terminal methio group $\left(-\mathrm{SCH}_{3}\right)$, from eukaryotes. Based on the production rate of methionine, Pilcher (2003) estimated the flux of $\mathrm{CH}_{3} \mathrm{SH}$ during the Archean to be $\sim 3 \times 10^{9} \mathrm{~mol} /$ year, or about $0.01 \mathrm{MDF} \mathrm{CH}_{3} \mathrm{SH}$. This estimate agrees with what one would get by simply scaling $\mathrm{CH}_{3} \mathrm{SH}$ production linearly with net primary productivity, as that is also estimated to have been 0.01 times the modern value (Kharecha et al., 2005). Because the Archean is our lone example of an anoxic planet, Pilcher's work serves as an estimate for the $S_{\text {org }}$ fluxes on extrasolar planets with anoxic surface conditions. However, these fluxes could vary if methionine (or some other Scontaining amino acid) was more or less prevalent in the planet's biota or if the biospheric productivity was different. Thus, in our primary suite of model runs, we parameterize the $S_{\text {org }}$ fluxes from methionine degradation, using values from 0 to 3000 times those estimated by Pilcher (2003) (this is equivalent to 0-30 MDF $\mathrm{S}_{\text {org }}$ ).

The direct production of $\mathrm{CH}_{3} \mathrm{SH}$ for metabolic purposes could lead to higher $S_{\text {org }}$ fluxes. Methanosarcina acetivorans, a methanogen, can produce $\mathrm{CH}_{3} \mathrm{SH}$ via the metabolic reaction $3 \mathrm{CO}+\mathrm{H}_{2} \mathrm{~S}+\mathrm{H}_{2} \mathrm{O} \rightarrow \mathrm{CH}_{3} \mathrm{SH}+2 \mathrm{CO}_{2}$ (Moran et al., 2008). In the rest of this manuscript, we will refer to this metabolism as "mercaptogenesis" and to the organisms that utilize it as "mercaptogens." Assuming substrate-limited (CO- $/ \mathrm{H}_{2} \mathrm{~S}-$ limited) conditions with no competition for substrates places an upper limit on mercaptogenesis. $\mathrm{CO}$ should build up to extremely high levels on planets with anoxic atmospheres unless consumed by biota (Zahnle, 1986; Kharecha et al., 2005); thus, $\mathrm{H}_{2} \mathrm{~S}$ is likely the limiting substrate on such planets. Estimates of the net primary productivity of S-consumers on Archean Earth vary by orders of magnitude, from $5 \times 10^{9} \mathrm{~mol} \mathrm{~S} /$ year (Kharecha et al., 2005) to $2 \times 10^{14} \mathrm{~mol}$ S/year (Canfield, 2005). Both estimates have caveats: the lower estimate did not include a complete $S$ cycle that allowed for recycling of $S$, and the upper estimate neglected inorganic sinks for $S$ such as metal-sulfide deposition. The former omission likely has a larger impact, so we used Canfield's estimate as an upper limit to $S$ utilization. If mercaptogens accounted for all $\mathrm{H}_{2} \mathrm{~S}$ used by metabolism, the range of the above $\mathrm{S}$ consumption estimates would correspond to $\mathrm{CH}_{3} \mathrm{SH}$ fluxes of $\sim 3 \times 10^{9}$ to $1 \times 10^{14}$ moles $\mathrm{CH}_{3} \mathrm{SH} /$ year, or $0.03-1000 \mathrm{MDF} \mathrm{CH}_{3} \mathrm{SH}$. Thus, $1000 \mathrm{MDF} \mathrm{CH}_{3} \mathrm{SH}$ is an upper limit to the $\mathrm{CH}_{3} \mathrm{SH}$ produced by mercaptogens on early Earth. The actual $\mathrm{CH}_{3} \mathrm{SH}$ production was likely much lower than this, due to competition for $\mathrm{CO}$ and $\mathrm{H}_{2} \mathrm{~S}$ from other metabolisms or from scavenging of $\mathrm{S}$ from the oceans by metal precipitates. On an extrasolar planet, the $\mathrm{CH}_{3} \mathrm{SH}$ production rate could be higher if the planet has larger volcanic $\mathrm{H}_{2} \mathrm{~S}$ flux rates. Constraining such fluxes may be possible via absorption features of volcanic gases in planetary spectra (Kaltenegger et al., 2010).

Unfortunately, no anoxic ocean model currently exists that includes biological S recycling and a complete accounting of oceanic S sources. Furthermore, no code exists that can model mercaptogens in the context of $\mathrm{CO}$-consuming methanogens and sulfur oxidizers that could compete for substrates. These problems might eventually be addressed by the development 
of ocean biogeochemistry codes with flexible chemistries and a wide variety of metabolisms. In the absence of such codes, we parameterized $\mathrm{CH}_{3} \mathrm{SH}$ fluxes from 1 to $100 \mathrm{MDF}$ to simulate a biosphere with $\mathrm{CO}$-consuming mercaptogens. Because the $\mathrm{CO}$ they consume would otherwise be used by methanogens, we decreased the biological $\mathrm{CH}_{4}$ flux in proportion to the biological $\mathrm{CH}_{3} \mathrm{SH}$ flux in these simulations. In this set of mercaptogenesis experiments with 1-100 MDF $\mathrm{CH}_{3} \mathrm{SH}$, we held the fluxes of the other $\mathrm{S}_{\text {org }}$ gases (DMS, DMDS, OCS, and $\mathrm{CS}_{2}$ ) constant at $1 \mathrm{MDF}$, because, unlike $\mathrm{CH}_{3} \mathrm{SH}$, these gases are not directly produced by this metabolism. To distinguish between the two sets of experiments, we label model simulations where we changed the flux of all $S_{\text {org }}$ gases with " $X$ MDF $S_{\text {org, }}$ " and label model simulations where we changed only the flux of $\mathrm{CH}_{3} \mathrm{SH}$ with "X $\mathrm{MDF} \mathrm{CH}_{3} \mathrm{SH}$."

Each set of $S_{\text {org }}$ boundary conditions was applied to planets orbiting stars of three different spectral types, following Segura et al. (2005). Specifically, we used timeaveraged spectra of the Sun, the active M dwarf AD Leo, and a model-generated $\mathrm{M}$ dwarf with a surface temperature of $3100 \mathrm{~K}$ and no chromosphere (Allard et al., 1997). This star, referred to as "T3100" in the remainder of this manuscript, is not presented as a physically meaningful case but rather as a low-UV-flux, end-member simulation. All stellar spectra were scaled such that the total energy flux at the top of the model planet's atmosphere was $1092 \mathrm{~W} / \mathrm{m}^{2}$, including radiation outside the bounds of our photochemical model wavelength grid. This is $80 \%$ of the flux Earth currently receives from the Sun, which is in line with the amount of energy the anoxic, Archean Earth received. Because the total energy flux received by the planet is the same, this is equivalent to assuming that the planet orbits within the habitable zone of that star. The resulting scaled stellar spectra are plotted in Fig. 1, as binned for use in the photochemical code.

\subsection{Radiative transfer code}

We used the line-by-line Spectral Mapping Atmospheric Radiative Transfer model (Meadows and Crisp, 1996; Crisp, 1997) to generate synthetic planetary spectra of our model planets. Spectra were computed by using the vertical mixing ratio profiles of $\mathrm{CH}_{3} \mathrm{SH}$, DMS, DMDS, OCS, $\mathrm{CS}_{2}, \mathrm{SO}_{2}, \mathrm{H}_{2} \mathrm{~S}$, $\mathrm{CH}_{4}, \mathrm{C}_{2} \mathrm{H}_{6}, \mathrm{CO}_{2}$, and $\mathrm{H}_{2} \mathrm{O}$ generated by the photochemical code. The underlying surface consisted of a $278 \mathrm{~K}$ global ocean with an emissivity of $\sim 1$, and we used the same assumed temperature structure applied in the photochemical model. The input stellar spectra and molecular absorption data were obtained from the Virtual Planetary Laboratory's online database (http://vpl.astro.washington.edu/spectra/ VPLSpectra/frontpage.htm) and include molecular line parameters from the HITRAN (Rothman et al., 2005) and PNNL databases (Sharpe et al., 2004).

We did not include any aerosols in our spectral model, so the model spectra shown here should be considered idealized "clear sky" simulations. However, we limited parameter space (see Boundary conditions, above) so that the atmospheric $\mathrm{CH}_{4} / \mathrm{CO}_{2}$ ratio was less than 0.1 , a condition for which thick organic haze layers will not form (Trainer et al., 2006; Haqq-Misra et al., 2008). $S_{8}$ and sulfate hazes were also limited by these conditions. Assuming Mie scattering, all $S_{8}$, hydrocarbon, and sulfate particles in our simulations had extinction optical depths less than 0.05 within the "IR win- dow" between 8.5 and $13 \mu \mathrm{m}$ in which most of the absorption features explored here appear. While organo-sulfate particles can form in sulfur-rich anoxic atmospheres (DeWitt et al., 2010), the optical properties of these particles have not yet been explored. Water clouds may also impact the spectra simulated here. For more on the effects of water clouds, see Robinson et al. (2011). We leave the exploration of aerosol and cloud effects for future studies.

\section{Results}

The habitable-zone planets around stars with lower surface temperatures receive proportionally fewer UV photons and more long-wavelength, low-energy photons (Fig. 1). This leads to lower photolysis rates on these planets, as there are fewer photons with the requisite energy to dissociate molecules. Figure $1 \mathrm{~b}$ illustrates this by showing the wavelengthdependent absorption cross section for $\mathrm{CH}_{3} \mathrm{SH}$ (Sharpe et al., 2004), along with the incident UV flux from the three different stars. Photolysis of $\mathrm{CH}_{3} \mathrm{SH}$ (and the other $\mathrm{S}_{\text {org }}$ species) generally occurs at wavelengths $<300 \mathrm{~nm}$, where the fluxes from the Sun, AD Leo, and T3100 differ by orders of magnitude. Except below $170 \mathrm{~nm}$, where the AD Leo habitable-zone planet receives the highest relative flux, the UV flux decreases dramatically going from the Sun to AD Leo to T3100. Because $\mathrm{CH}_{3} \mathrm{SH}$ photolysis occurs mostly in the $200-300 \mathrm{~nm}$ region, its photolysis rate follows this same pattern. The same holds true for other gases, for example, $\mathrm{H}_{2} \mathrm{O}$, whose photolysis creates highly reactive radicals that destroy $S_{\text {org }}$.

\subsection{Production and loss of $S_{\text {org }}$ species}

We define a standard Archean model with the general boundary conditions above along with $1 \mathrm{MDF} \mathrm{S}_{\text {org }}$ and 1 MDF $\mathrm{CH}_{4}$. The largest $\mathrm{S}_{\text {org }}$ sinks in this simulation were the following reactions:

$$
\begin{aligned}
& \mathrm{C}_{2} \mathrm{H}_{6} \mathrm{~S}_{2}+\mathrm{O} \rightarrow \mathrm{CH}_{3}+\mathrm{SO}+\mathrm{CH}_{3} \mathrm{~S} \\
& \mathrm{OCS}+h \nu \rightarrow \mathrm{CO}+\mathrm{S} \\
& \mathrm{C}_{2} \mathrm{H}_{6} \mathrm{~S}+\mathrm{O} \rightarrow \mathrm{CH}_{3}+\mathrm{CH}_{3}+\mathrm{SO} \\
& \mathrm{CH}_{3} \mathrm{SH}+\mathrm{O} \rightarrow \mathrm{CH}_{3}+\mathrm{HSO}
\end{aligned}
$$

These reactions outpaced other net $S_{\text {org }}$ sinks by at least an order of magnitude. Thus, the major sink for $S_{\text {org }}$ in our model was reaction with $\mathrm{O}$, and the major by-products were $\mathrm{CH}_{3}$ and oxidized sulfur species-SO and HSO. The major source of $\mathrm{O}$ atoms to the atmosphere was photolysis of major atmospheric components (in an anoxic atmosphere, $\mathrm{CO}_{2}$, $\mathrm{SO}_{2}$, and $\mathrm{H}_{2} \mathrm{O}$ ), and the inventory of $\mathrm{O}$ atoms decreased when the flux of UV photons to the atmosphere was diminished.

On the model planet orbiting T3100, the biggest sinks for $S_{\text {org }}$ species were the following reactions:

$$
\begin{aligned}
& \mathrm{OCS}+h \nu \rightarrow \mathrm{CO}+\mathrm{S} \\
& \mathrm{OCS}+\mathrm{S} \rightarrow \mathrm{CO}+\mathrm{S}_{2} \\
& \mathrm{CH}_{3} \mathrm{SH}+h \nu \rightarrow \mathrm{CH}_{3}+\mathrm{HS} \\
& \mathrm{C}_{2} \mathrm{H}_{6} \mathrm{~S}_{2}+\mathrm{O} \rightarrow \mathrm{CH}_{3}+\mathrm{SO}+\mathrm{CH}_{3} \mathrm{~S} \\
& \mathrm{CH}_{3} \mathrm{SH}+\mathrm{O} \rightarrow \mathrm{CH}_{3}+\mathrm{HSO}
\end{aligned}
$$

In the model simulations around these stars, the lack of UV photons entering the atmosphere led to a lack of $\mathrm{O}$ radicals in 

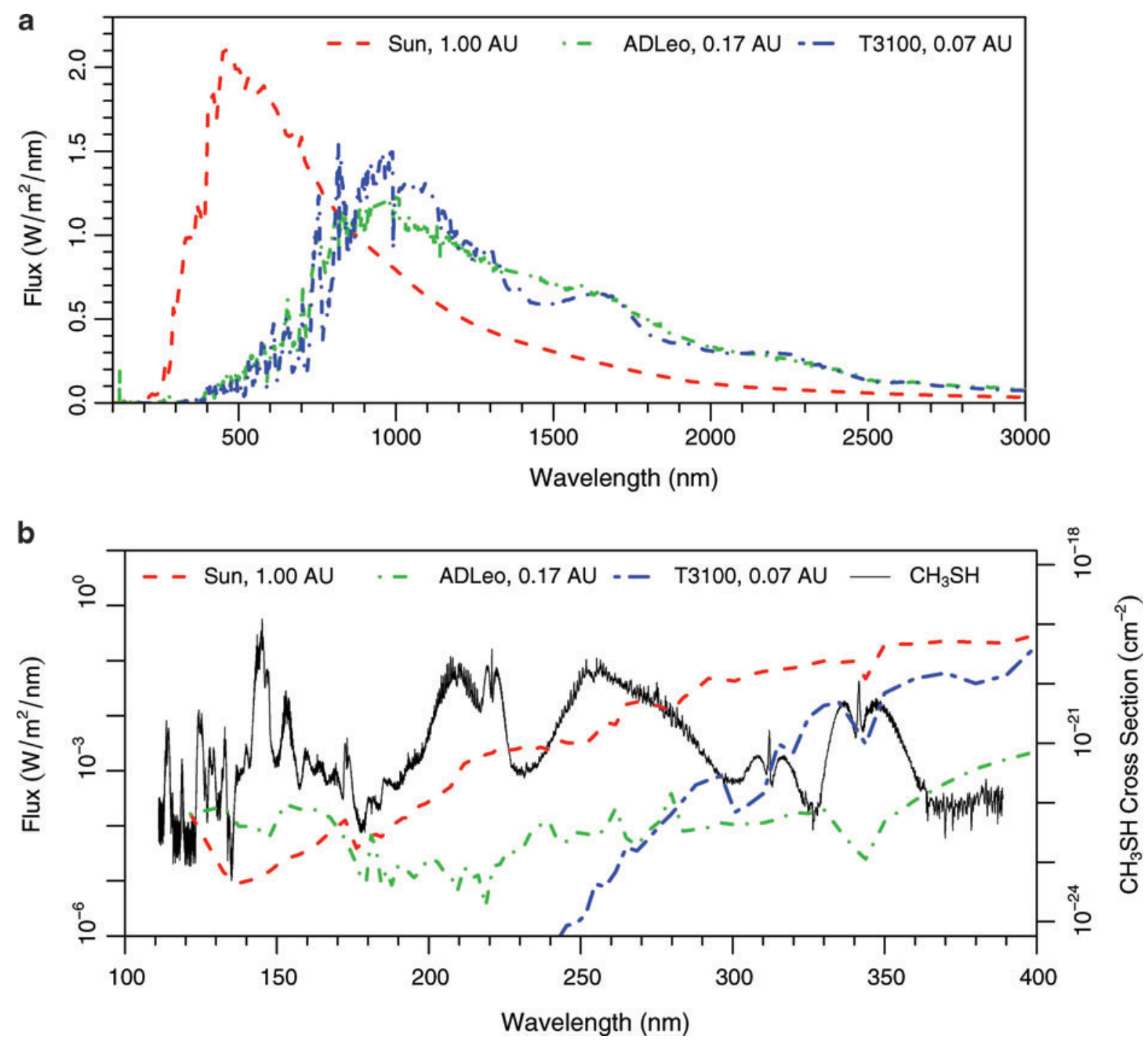

FIG. 1. (a) The stellar energy distribution at a planet receiving the same amount of total energy flux that the Earth received $\sim 2.5$ billion years ago for three different stars: the Sun, AD Leo, and T3100 (a model M dwarf that has no chromosphere). (b) The bottom panel is an expansion of the UV region of the top panel, with a logarithmic $y$ axis. The bottom panel also shows the absorption cross section of $\mathrm{CH}_{3} \mathrm{SH}$, units for which are on the right $y$ axis (also logarithmic). Color images available online at www.liebertonline.com/ast

the atmosphere. This caused a slower destruction rate of the $S_{\text {org }}$ gases and shifted the main by-products of $S_{\text {org }}$ photochemistry to carbon monoxide (CO) and reduced sulfur species $\left(\mathrm{S}, \mathrm{S}_{2}\right.$, and $\left.\mathrm{H}_{2} \mathrm{~S}\right)$. The planets orbiting AD Leo were between these two end-member cases for atomic $\mathrm{O}$ production. As a result, the by-products of $S_{\text {org }}$ chemistry on planets around $M$ dwarfs were a mix of oxidized and reduced sulfur species.

\subsection{Atmospheric profiles}

Results from nine photochemical model runs are shown in Figs. 2 and 3. Each figure contains a $3 \times 3$ grid of panels with decreasing UV flux (Sun, AD Leo, T3100) from left to right and increasing organic sulfur gases (0 MDF $S_{\text {org }}$, our

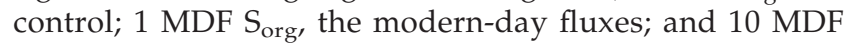
$\mathrm{CH}_{3} \mathrm{SH}$, corresponding to a biosphere containing mercaptogens) from top to bottom. Figure 2 shows the calculated mixing ratio profiles of the major $S_{\text {org }}$ species along with
$\mathrm{SO}_{2}$ and $\mathrm{H}_{2} \mathrm{~S}$, while Fig. 3 shows the calculated vertical profiles of $\mathrm{H}_{2} \mathrm{O}, \mathrm{CH}_{4}, \mathrm{C}_{2} \mathrm{H}_{6}, \mathrm{H}_{2}$, and $\mathrm{O}_{2}$. The profiles generated with $0 \mathrm{MDF}_{\text {org }}$ are our control experiments, as this boundary condition is equivalent to assuming no biological $\mathrm{S}_{\text {org }}$ production. In these cases, the atmospheric mixing ratios of all $S_{\text {org }}$ gases were extremely low.

For models with the modern-day $S_{\text {org }}$ flux, near-surface mixing ratios of DMS built up to at least $\sim 10 \mathrm{ppt}\left(10^{-11}\right)$ for all three stellar types. These relatively low concentrations are due to higher photolysis rates in the absence of an $\mathrm{O}_{2} / \mathrm{O}_{3}$ UV shield. For the T3100 model planet, DMDS and $\mathrm{CH}_{3} \mathrm{SH}$ peaked above $100 \mathrm{ppb}\left(10^{-7}\right)$. The shapes of the $S_{\text {org }}$ profiles also changed as a function of star type, as the sulfur gases remained well mixed to higher altitudes in the low-UV-flux models, further increasing the total column depths of the $S_{\text {org }}$ species. $\mathrm{C}_{2} \mathrm{H}_{6}$ concentrations also increased when surface $\mathrm{S}_{\text {org }}$ production was included, because of additional production of $\mathrm{CH}_{3}$ radicals (Fig. 3). 


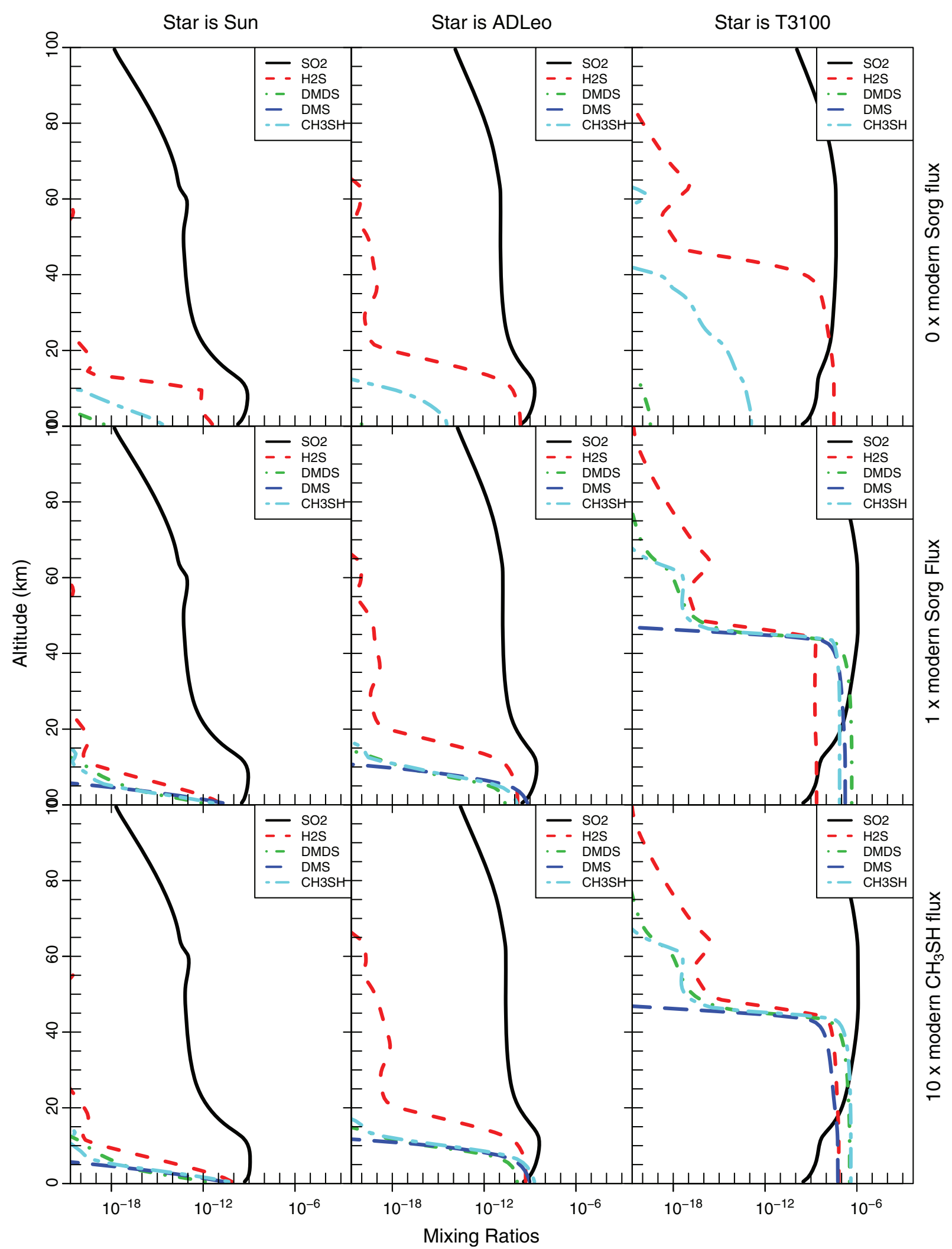

FIG. 2. These nine panels each show model-predicted vertical profiles of the mixing ratios of the organic sulfur species. Panels toward the left are for planets orbiting stars with greater UV radiation, and panels toward the bottom are for planets with higher biological $S_{\text {org }}$ production. The $S_{\text {org }}$ mixing ratios increase with higher ground $S_{\text {org }}$ fluxes (bottom panels) and with lower UV radiation (right panels). Color images available online at www.liebertonline.com/ast 


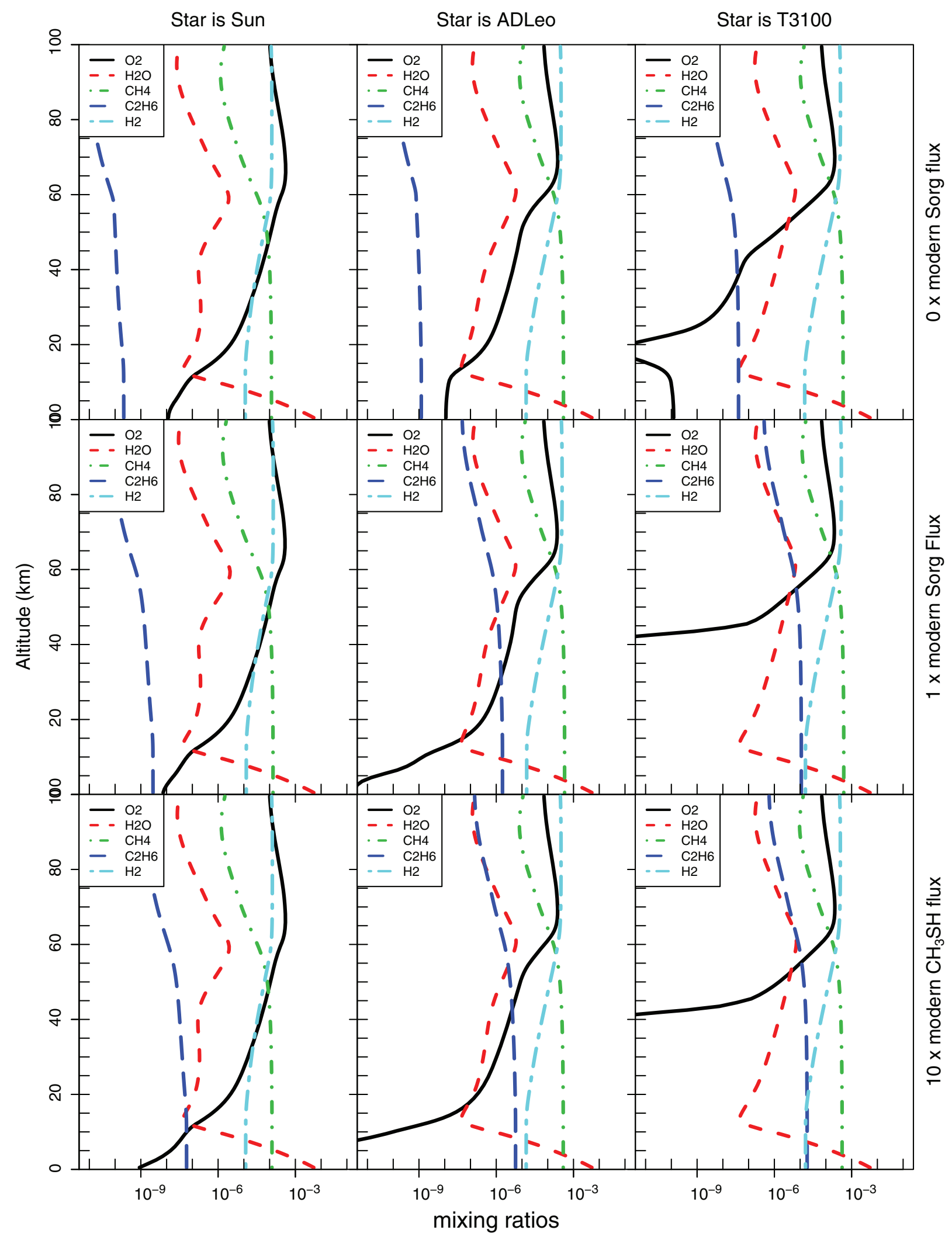

FIG. 3. These nine panels each show model-predicted vertical profiles of the mixing ratios of the greenhouse gases in our climate and line-by-line radiative transfer models. Panels on the left are for planets orbiting stars with greater UV radiation, and panels on the bottom are for planets with higher biological $\mathrm{S}_{\text {org }}$ production. $\mathrm{H}_{2} \mathrm{O}$ and $\mathrm{CO}_{2}$ concentrations are identical in all model runs, while $\mathrm{CH}_{4}$ concentrations vary only modestly between simulations. Note the increase in $\mathrm{C}_{2} \mathrm{H}_{6}$ concentrations on planets with higher $\mathrm{S}_{\text {org }}$ fluxes or lower UV radiation, or both. Color images available online at www.liebertonline.com/ast 
As expected, increasing the $\mathrm{CH}_{3} \mathrm{SH}$ flux to $10 \mathrm{MDF}$ while keeping the rest of the $S_{\text {org }}$ gases at 1 MDF (the mercaptogen experiments) resulted in a further increase in all $S_{\text {org }}$ mixing ratios (Fig. 2). This increase was most pronounced in $\mathrm{CH}_{3} \mathrm{SH}$ and in DMDS and was greatest on planets receiving relatively low UV radiation. $\mathrm{C}_{2} \mathrm{H}_{6}$ concentrations also increased with these higher $\mathrm{CH}_{3} \mathrm{SH}$ fluxes (Fig. 3), despite the fact that we decreased the $\mathrm{CH}_{4}$ fluxes in these simulations so that the total flux of $\mathrm{CH}_{3}$ groups to the atmosphere remained constant.

\subsection{Spectra}

To illustrate where each of the gases plotted in Figs. 2 and 3 are spectrally active, we present sensitivity spectra of the model planet with $30 \mathrm{MDF} \mathrm{CH}_{3} \mathrm{SH}$ orbiting AD Leo (Fig. 4). We generated Fig. 4 by running a full spectral model (shown as a black curve) and then subsequent model runs with one gas removed in each run. These sensitivity spectra are not self-consistent atmospheres; rather, they are tools to determine what gases are causing certain absorption features in the full spectral model. The spectral regions in which a sensitivity spectrum for a particular gas differs from the planet's complete spectrum show where that gas absorbs. For example, the effects of $\mathrm{H}_{2} \mathrm{O}$ are clearly seen (difference between black and gray curves) from 5 to $7 \mu \mathrm{m}$ and longward of $17 \mu \mathrm{m}$. Likewise, $\mathrm{CO}_{2}$ absorption features (difference between black and brown curves) are present from 9 to $11 \mu \mathrm{m}$ and from 12 to $19 \mu \mathrm{m}, \mathrm{CH}_{4}$ absorption is present from 6 to $9 \mu \mathrm{m}$, and $\mathrm{C}_{2} \mathrm{H}_{6}$ has a deep absorption feature from 11 to $13 \mu \mathrm{m}$. The distinguishable $\mathrm{S}_{\text {org }}$ absorption features include those caused by $\mathrm{CH}_{3} \mathrm{SH}$ from 9 to $11 \mu \mathrm{m}$ and by DMDS from 10 to $11 \mu \mathrm{m}$.

Model spectra from 4 to $20 \mu \mathrm{m}$ are presented at a spectral resolution of $R(\lambda / \Delta \lambda) \sim 50$ in Fig. 5. This resolution is consistent with the requirement goal for the Terrestrial Planet Finder Interferometer (TPF-I), a first-generation thermal-IR planet characterization mission (Lawson et al., 2007). For the simulations of a mercaptogen biosphere on a planet with a spectrum of the Sun or AD Leo, the greatest remotely observable difference was the $\mathrm{C}_{2} \mathrm{H}_{6}$ absorption feature between 11 and $13 \mu \mathrm{m}$, the strength of which increases at higher $\mathrm{CH}_{3} \mathrm{SH}$ fluxes $\left(30 \times\right.$ modern $\mathrm{CH}_{3} \mathrm{SH}$ flux). This feature became more prevalent if we increased the flux of the other $S_{\text {org }}$ gases $\left(30 \times\right.$ modern $S_{\text {org }}$ flux) or if we decreased the UV radiation reaching the planet (bottom panel). The model simulations with these deeper $\mathrm{C}_{2} \mathrm{H}_{6}$ features also exhibited enhanced absorption features from 8.5 to $11 \mu \mathrm{m}$ caused by DMDS.

$\mathrm{H}_{2} \mathrm{~S}$ fluxes are unlikely to cause false positives. $\mathrm{H}_{2} \mathrm{~S}$ had a large spectral influence only on planets with extremely large $\mathrm{H}_{2} \mathrm{~S}$ fluxes $\left(1000 \times \mathrm{H}_{2} \mathrm{~S} \mathrm{MDF}\right)$ orbiting stars with extremely low UV radiation (T3100). Except for these end-member cases, we do not expect $\mathrm{H}_{2} \mathrm{~S}$ to provide a false negative for the other absorption features discussed here.

\section{Discussion}

Several trends from our photochemical simulations (Figs. 2 and 3) have implications for the interpretation of future exoplanetary spectra. As the stellar UV flux to the planet decreases, the ground-level mixing ratios and altitudinal extent of $S_{\text {org }}$ species increase. The same effects can also be caused by increases to the $S_{\text {org }}$ surface fluxes. Both trends can be explained by an increase in the ratio of $S_{\text {org }}$ sources to $S_{\text {org }}$ sinks. The main sources of $S_{\text {org }}$ to the atmosphere are the biogenic surface fluxes; an increase in these raises the source/sink ratio. The two main sinks of $S_{\text {org }}$ species are direct photolysis and reaction with radicals such as $\mathrm{OH}$ and $\mathrm{O}$ that themselves are by-products of photochemical reactions. The decrease in UV radiation slows all photolysis and therefore decreases the sinks for $S_{\text {org }}$ species.

The other robust trend in the photochemical simulations is an increase in $\mathrm{C}_{2} \mathrm{H}_{6}$ with increasing $\mathrm{S}_{\text {org }}$ fluxes and with decreasing UV radiation. Increasing $S_{\text {org }}$ fluxes increases the source of $\mathrm{CH}_{3}$ radicals that combine to form $\mathrm{C}_{2} \mathrm{H}_{6}$. Decreases in UV fluxes lead to lower $\mathrm{C}_{2} \mathrm{H}_{6}$ photolysis rates, lower concentrations of $\mathrm{C}_{2} \mathrm{H}_{6}$-destroying radicals, and smaller sinks for $\mathrm{C}_{2} \mathrm{H}_{6}$.

$\mathrm{C}_{2} \mathrm{H}_{6}$ has not previously been identified as a potential biosignature for anoxic atmospheres, although most concepts for mid-IR exoplanet characterization missions already include plans to detect $\mathrm{CH}_{4}$ by looking for its absorption feature centered near $7.7 \mu \mathrm{m}$ (Lawson et al., 2007). According to our model simulations, $\mathrm{C}_{2} \mathrm{H}_{6}$ detection would require an interferometer with a spectral resolution of $\lambda / \Delta \lambda \sim 20$ and a $\mathrm{S} / \mathrm{N} \sim 15$ in the $11-13 \mu \mathrm{m}$ range to resolve the distinctive band profile for this gas. Such a mission could discriminate at a $3 \sigma$ level between $\mathrm{C}_{2} \mathrm{H}_{6}$ produced by the model with the modern-day $S_{\text {org }}$ flux and the model with no $S_{\text {org }}$ flux, for a planet around an $\mathrm{M}$ dwarf similar to AD Leo.

$\mathrm{C}_{2} \mathrm{H}_{6}$ concentrations can be enhanced both by increased $\mathrm{S}_{\text {org }}$ concentrations and by increased $\mathrm{CH}_{4}$. Because $\mathrm{CH}_{4}$ can have an abiogenic source, $\mathrm{CH}_{4}$-derived $\mathrm{C}_{2} \mathrm{H}_{6}$ could be abiogenic in origin. Figure 5 shows low-resolution $(R \sim 50)$ spectra with high $\mathrm{C}_{2} \mathrm{H}_{6}$ concentrations arising from either high $\mathrm{S}_{\text {org }}$ fluxes or high $\mathrm{CH}_{4}$ fluxes. Models that have higher $\mathrm{S}_{\text {org }}$ fluxes have higher $\mathrm{C}_{2} \mathrm{H}_{6}$ concentrations and a deeper $\mathrm{C}_{2} \mathrm{H}_{6}$ absorption feature between 11 and $13 \mu \mathrm{m}$. Similarly, models that have higher $\mathrm{CH}_{4}$ fluxes also have increased $\mathrm{C}_{2} \mathrm{H}_{6}$ concentrations and more absorption between 11 and $13 \mu \mathrm{m}$. However, models that achieve $\mathrm{C}_{2} \mathrm{H}_{6}$ buildup through increased $\mathrm{CH}_{4}$ fluxes also exhibit a detectable increase in the $\mathrm{CH}_{4}$ concentrations in the atmosphere: there was a doubling in the nearsurface $\mathrm{CH}_{4}$ mixing ratios when the $\mathrm{CH}_{4}$ fluxes were increased to $1.5 \mathrm{MDF}$, and another doubling when the $\mathrm{CH}_{4}$ fluxes were increased to 2.0 MDF. These increased $\mathrm{CH}_{4}$ concentrations caused significantly more absorption between 8 and $9 \mu \mathrm{m}$. In other words, changes in the absorption by $\mathrm{CH}_{4}$ could potentially allow us to discriminate between the spectra with "abiogenic, $\mathrm{CH}_{4}$-derived $\mathrm{C}_{2} \mathrm{H}_{6}$ " and the spectra with "biogenic, $\mathrm{S}_{\text {org }}$-derived $\mathrm{C}_{2} \mathrm{H}_{6}$." Thus, an exoplanet characterization mission that can measure the depths of the $\mathrm{CH}_{4}$ and $\mathrm{C}_{2} \mathrm{H}_{6}$ absorption features accurately enough to estimate the $\mathrm{C}_{2} \mathrm{H}_{6} / \mathrm{CH}_{4}$ ratio may be able to determine whether biological $\mathrm{S}_{\text {org }}$ production contributes to the source of $\mathrm{C}_{2} \mathrm{H}_{6}$.

These above differences in $\mathrm{CH}_{4}$ absorption depths in biological and abiological model simulations are the result of higher $\mathrm{C}_{2} \mathrm{H}_{6} / \mathrm{CH}_{4}$ ratios in models with biological $\mathrm{S}_{\text {org }}$ fluxes. These fluxes caused an increase in atmospheric $\mathrm{CH}_{3}$ groups, which in turn increased the atmospheric $\mathrm{C}_{2} \mathrm{H}_{6} / \mathrm{CH}_{4}$ ratio. Thus, for a given amount of $\mathrm{C}_{2} \mathrm{H}_{6}$, the $\mathrm{CH}_{4}$ concentrations were lower in models with higher $\mathrm{S}_{\text {org }}$ fluxes. (The converse is also true; for a given $\mathrm{CH}_{4}$ concentration, models with higher $\mathrm{S}_{\text {org }}$ fluxes exhibited higher $\mathrm{C}_{2} \mathrm{H}_{6}$ concentrations.) This effect could be augmented by inclusion of other 

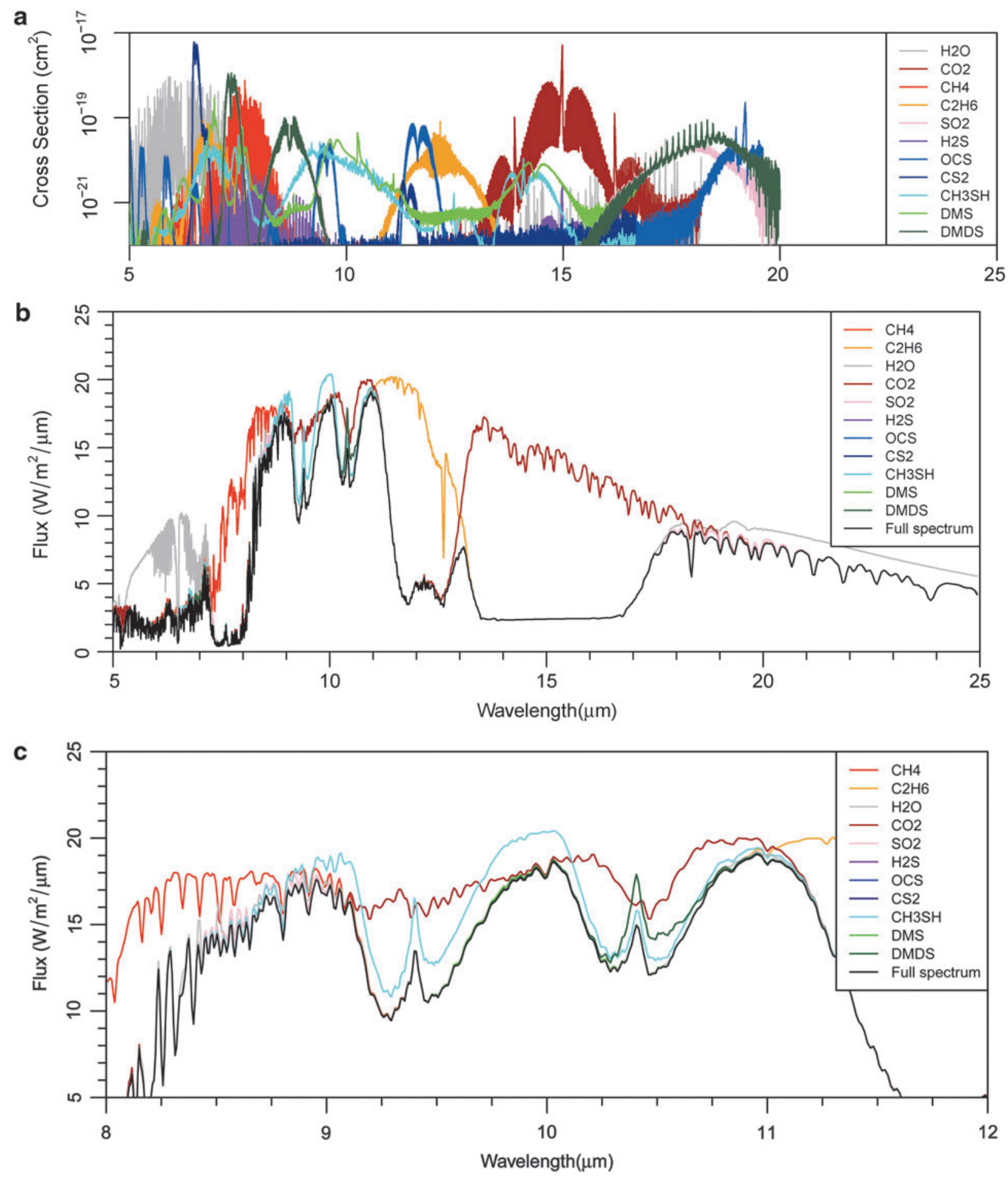

FIG. 4. The top panel shows the absorption cross sections for the gases included in our spectral model. The middle and bottom panels show the simulated spectra for a simulation of a planet with $30 \mathrm{MDF} \mathrm{S}_{\text {org }}$ orbiting AD Leo. The black line shows the full model spectrum, including the influence of all the gases in our line-by-line radiative transfer model. The colored lines show model spectra in which one gas is removed from the line-by-line radiative transfer model, with lines of the same color showing the absorption cross-section spectrum for that gas in the top pane. For example, the gray line shows the spectrum with the radiative influence of $\mathrm{H}_{2} \mathrm{O}$ removed from the model. The bottom panel shows a zoom-in on the "infrared window" between 8.5 and $11 \mu \mathrm{m}$.

biological $\mathrm{CH}_{3} \mathrm{X}$ species, such as $\mathrm{CH}_{3} \mathrm{Cl}$, that were not included in these simulations.

In addition to the influence of $\mathrm{S}_{\text {org }}$ species on the $\mathrm{C}_{2} \mathrm{H}_{6}$ feature, several other features were caused directly by the presence of the $S_{\text {org }}$ in the model atmospheres: absorption just shortward of $7 \mu \mathrm{m}$ by DMS, absorption just longward of $7 \mu \mathrm{m}$ by DMDS, absorption from 8.5 to $9.5 \mu \mathrm{m}$ by DMDS, and absorption between 9 and $11 \mu \mathrm{m}$ by DMDS and $\mathrm{CH}_{3} \mathrm{SH}$. When present, these features created a continuous, but not constant, increase in absorption from $6 \mu \mathrm{m}$ all the way to the $\mathrm{C}_{2} \mathrm{H}_{6}$ 

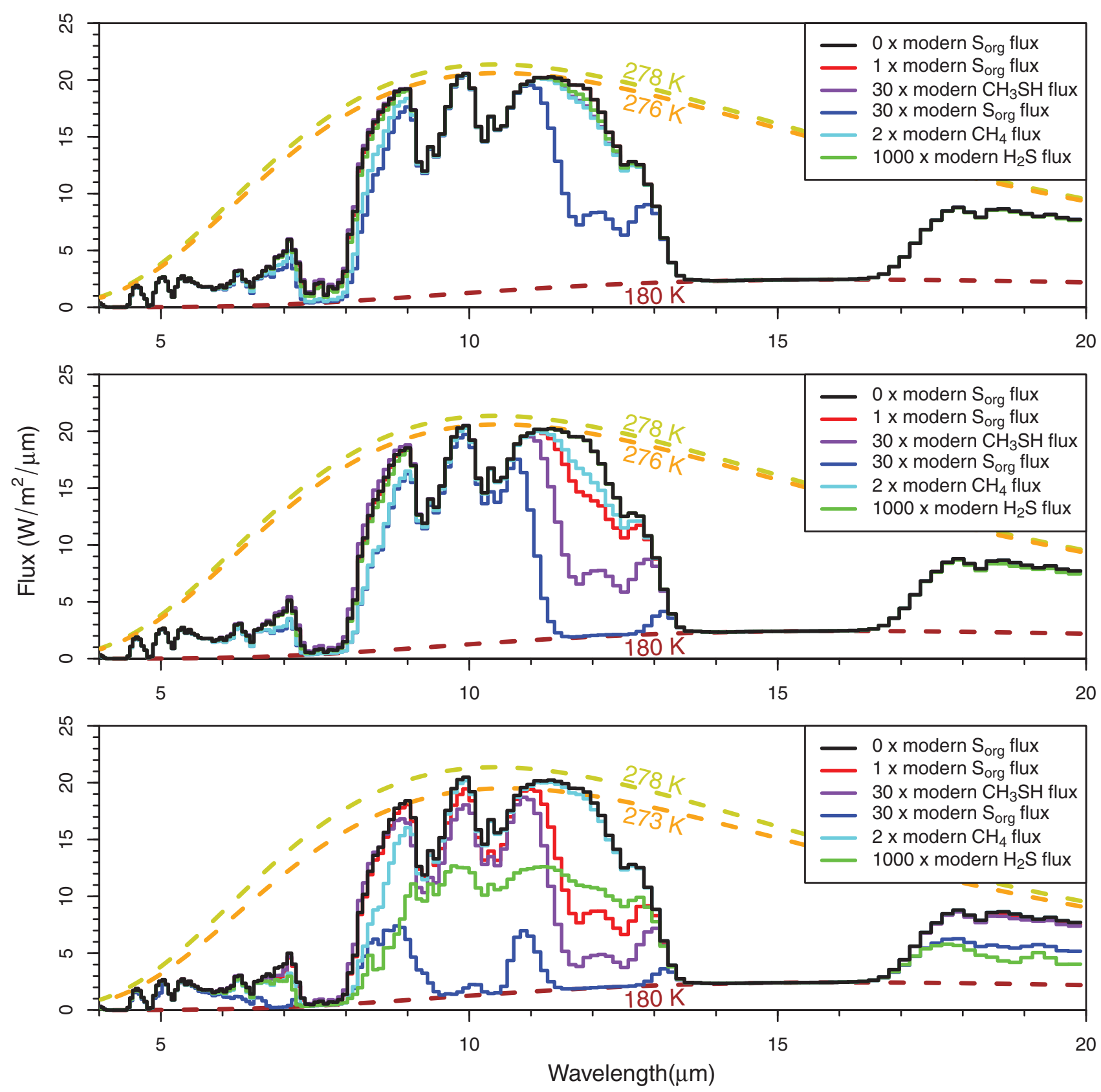

FIG. 5. Spectra for planets around the Sun (top panel), AD Leo (middle panel), and T3100, a model M dwarf with no chromosphere (bottom panel), all at a spectral resolution of $\lambda / \Delta \lambda \sim 50$. The black curve is a spectrum for a planet with $0 S_{\text {org }}$ flux. The red and blue lines show model spectra for planets with 1 and 30 times the modern $S_{\text {org }}$ fluxes. The purple lines show spectra for planets with 30 times the modern day flux of $\mathrm{CH}_{3} \mathrm{SH}$ and 0.65 times the modern day flux of $\mathrm{CH}_{4}$. The cyan lines show spectra with 2 times the modern day flux of $\mathrm{CH}_{4}$ and $0 \mathrm{~S}_{\text {org }}$ flux. The green lines show spectra with 1000 times the modern day flux of $\mathrm{H}_{2} \mathrm{~S}$ and $0 \mathrm{~S}_{\text {org }}$ flux. The goldenrod, orange, and brown dashed lines represent the Planck function for an object at $278 \mathrm{~K}$ (the surface temperature), 276 or $273 \mathrm{~K}$ (the highest "color temperature" for the " 1 modern $\mathrm{S}_{\text {org }}$ flux" spectrum), and $180 \mathrm{~K}$ (the stratospheric temperature).

feature at $11 \mu \mathrm{m}$. Thus, they have a significant impact across a wide wavelength range. However, these features only appeared in model simulations with extremely low UV fluxes (the T3100 case) or in simulations with at least 30-fold increases in the flux rate of all $S_{\text {org }}$ gases. On planets around more active stars, these features would only be detectable if the biosphere is much more productive than Earth's biosphere or if the organisms living on the planet have high concentrations of sulfur in their proteins. Even planets with an active mercaptogen community would not produce these features unless that community produces $\mathrm{CH}_{3} \mathrm{SH}$ at a rate that is greater than 30 times the modern-day $\mathrm{CH}_{3} \mathrm{SH}$ flux from the oceans.

Additional confusion in interpreting potential arises from the influence of surface temperature. Discriminating between planets with absorption by $S_{\text {org }}$ species and planets with lower surface temperatures may prove problematic, as the $\mathrm{S}_{\text {org }}$ gases all absorb in the 8-12 $\mu \mathrm{m}$ "atmospheric window" wavelength region. This is a part of the spectrum that some 
have suggested could be used to discern surface temperatures, because on modern-day Earth that region is the most transparent to the IR radiation emitted by the surface of Earth. However, an increase in greenhouse gases that absorb photons in this region (including $S_{\text {org }}$ species) will increase its opacity, thereby decreasing the effectiveness with which the surface temperature can be ascertained.

The quantitative effect of $S_{\text {org }}$ absorption on inferred planetary temperature is shown by the dashed curves in Fig. 5 . Here the model spectra, which are cloud free, have been degraded to the spectral resolution goal for TPF-I and are shown with blackbody spectra at three temperatures: (1) $180 \mathrm{~K}$, the stratospheric temperature in our model (drawn in brown); (2) $278 \mathrm{~K}$, the surface temperature in our model (drawn in goldenrod); and (3) either $276 \mathrm{~K}$ (top, middle) or $273 \mathrm{~K}$ (bottom), the maximum temperature derived for $1 \mathrm{MDF} \mathrm{S}_{\text {org }}$ case within the window region of the model spectrum (drawn in orange). Figure 5 shows that the $S_{\text {org }}$ gas absorption, in addition to weak water vapor absorption, increases the opacity of the atmosphere in the atmospheric window sufficiently that the majority of the radiation sensed comes from higher, colder regions of the planet's troposphere. The discrepancy between actual surface temperature $(278 \mathrm{~K})$ and maximum observed temperature is as much as $8 \mathrm{~K}$ for the highest $S_{\text {org }}$ fluxes and lowest UV fluxes. This will increase the planet's greenhouse effect but decrease the effectiveness with which the surface temperature can be sensed remotely. This effect is from atmospheric absorption alone and does not account for the atmospheric column-truncating effects of clouds or hazes, which for an unresolved Earth-like planet can further reduce the measured brightness temperature in the window region.

Obtaining the best possible estimates of planetary surface temperatures for extrasolar planets of unknown composition will therefore require sufficient spectral wavelength range and resolution to identify non-Earth-like atmospheric window regions, and good estimates of planetary composition and the presence of cloud or aerosol cover. These measurements, combined with atmospheric modeling, will be crucial for understanding limitations on planetary temperature retrieval from MIR spectra for planets with atmospheric characteristics unlike those of modern Earth. For anoxic atmospheres, it is important to be able to detect $S_{\text {org }}$ absorption features at wavelengths shortward of the window region. Absorption by DMS and DMDS between 6 and $9 \mu \mathrm{m}$ provides an extra constraint on the abundance of these gases. Similarly, the $\mathrm{C}_{2} \mathrm{H}_{6}$ feature could be used in conjunction with photochemical models to further constrain the $S_{\text {org }}$ flux rates. The atmospheric $S_{\text {org }}$ inventory could then be input to a climate model to calculate self-consistent surface temperatures and spectra. A fairly comprehensive characterization of an anoxic atmosphere could therefore be achieved with spectra from 6 to $13 \mu \mathrm{m}$ (and preferably down to $5 \mu \mathrm{m}$ and out to $20 \mu \mathrm{m}$ to help constrain water abundances) at a spectral resolution of at least 20 and a S/N greater than 15 . These baseline parameters are consistent with the current requirement goals for the TPF-I mission concept.

\section{Conclusions}

In this paper, we have shown that an anoxic biosphere could be detected over interstellar distances by searching for organic S species produced by biology. On planets orbiting
Sun-type stars, $\mathrm{S}_{\text {org }}$ fluxes at 30 times modern-day levels could be detected in the form of elevated $\mathrm{C}_{2} \mathrm{H}_{6} / \mathrm{CH}_{4}$ ratios that are a photochemical by-product of $\mathrm{S}_{\text {org }}$ gases. On planets around $\mathrm{M}$ dwarfs such as AD Leo, detection of heightened $\mathrm{C}_{2} \mathrm{H}_{6} / \mathrm{CH}_{4}$ ratios is possible at present-day $\mathrm{S}_{\text {org }}$ fluxes. Features caused directly by $S_{\text {org }}$ gases may be observable on planets that have much higher $S_{\text {org }}$ fluxes or on planets orbiting $\mathrm{M}$ dwarfs that exhibit low amounts of stellar activity, or both. An important caveat to this work is that aerosols, including water clouds, hydrocarbon aerosols, sulfate aerosols, and $\mathrm{S}_{8}$ particles, were not considered in the spectral portion of this study but may impact the ability to detect these species.

The detection of any of these features will require an instrument with spectral resolution $R>20$, broad coverage of the IR spectrum $(6-14 \mu \mathrm{m})$, and low total noise levels (S/ $\mathrm{N}>15$ or noise $\left.<1 \mathrm{~W} / \mathrm{m}^{2} / \mu \mathrm{m}\right)$. Current expected performance levels for TPF-I meet these requirements (Lawson et al., 2007). The use of models to interpret the spectra will also be required in order to separate the effects of surface temperature, organic sulfur gases, and other atmospheric constituents on the planetary spectrum.

Despite the difficulties involved, the benefits offered by such a search are considerable. By including organic sulfur species in our repertoire of remotely detectable biosignatures, the detection of life on some planets may also come with rudimentary lessons on the composition of that planet's biosphere. Thus, this work supports exoplanet characterization missions with a wavelength range and spectral resolution sufficient to detect $\mathrm{C}_{2} \mathrm{H}_{6}$ and the organic sulfur gases discussed above.

\section{Acknowledgments}

The quality of this manuscript was improved by the useful critiques of three reviewers. This work was performed as part of the NASA Astrobiology Institute's Virtual Planetary Laboratory, supported by the National Aeronautics and Space Administration through the NASA Astrobiology Institute under solicitation No. NNH05ZDA001C. Additional support was provided by the Pennsylvania State Astrobiology Research Center, supported by the National Aeronautics and Space Administration through the NASA Astrobiology Institute under grant number NNA09DA76A. S.D.D.-G. acknowledges additional support from the Astrobiology NSF IGERT grant at the University of Washington. M.C. and S.D.D.-G. acknowledge additional support from the NASA Postdoctoral Program. J.F.K. acknowledges additional support from NASA's Exobiology and Evolutionary Biology Program.

\section{Abbreviations}

DMS, dimethyl sulfide; DMDS, dimethyl disulfide; MDF, modern-day flux; S/N, signal-to-noise ratio; TPF-I, Terrestrial Planet Finder Interferometer.

\section{References}

Adachi, H., Basco, N., and James, D.G.L. (1981) The acetyl radicals $\mathrm{CH}_{3} \mathrm{CO}^{-}$and $\mathrm{CD}_{3} \mathrm{CO}^{-}$studied by flash photolysis and kinetic spectroscopy. International Journal of Chemical Kinetics 13:1251-1276. 
Albers, E.A., Hoyermann, K., Wagner, H.G., and Wolfrum, J. (1969) Study of the reaction of ammonia with oxygen atoms. Symposium (International) on Combustion 12:313-321.

Allard, F., Hauschildt, P.H., Alexander, D.R., and Starrfield, S. (1997) Model atmospheres of very low mass stars and brown dwarfs. Annu Rev Astron Astrophys 35:137-177.

Allen, M., Yung, Y.L., and Gladstone, G.R. (1992) The relative abundance of ethane to acetylene in the jovian stratosphere. Icarus 100:527-533.

Amano, A., Yamada, M., Hashimoto, K., and Sugiura, K. (1983) Kinetic feature of the reaction between methanethiol and hydrogen atoms. Nippon Kagaku Kaishi 12:385-393.

Anastasi, C., Broomfield, M., Nielsen, O.J., and Pagsberg, P. (1991) Ultraviolet absorption spectra and kinetics of $\mathrm{CH}_{3} \mathrm{~S}$ and $\mathrm{CH}_{2} \mathrm{SH}$ radicals. Chem Phys Lett 182:643-648.

Arthur, N.L. and Lee, M. (1976) Reactions of methyl radicals. I. Hydrogen abstraction from dimethyl sulphide. Aust J Chem 29:1483-1492.

Ashfold, M.N.R., Fullstone, M.A., Hancock, G., and Ketley, G.W. (1981) Singlet methylene kinetics: direct measurements of removal rates of $\tilde{a}^{1} \mathrm{~A}_{1}$ and $\tilde{\mathrm{b}}^{1} \mathrm{~B}_{1} \mathrm{CH}_{2}$ and $\mathrm{CD}_{2}$. Chem Phys 55:245257.

Atkinson, R., Baulch, D.L., Cox, R.A., Hampson, J.R.F., Kerr, J.A., and Troe, J. (1989) Evaluated kinetic and photochemical data for atmospheric chemistry: supplement III. IUPAC Subcommittee on Gas Kinetic Data Evaluation for Atmospheric Chemistry. Journal of Physical and Chemical Reference Data 18:881-1097.

Atkinson, R., Baulch, D.L., Cox, R.A., Crowley, J.N., Hampson, R.F., Hynes, R.G., Jenkin, M.E., Rossi, M.J., and Troe, J. (2004) Evaluated kinetic and photochemical data for atmospheric chemistry: Volume I-gas phase reactions of $\mathrm{O}_{X}, \mathrm{HO}_{X}, \mathrm{NO}_{X}$ and $\mathrm{SO}_{\mathrm{X}}$ species. Atmos Chem Phys 4:1461-1738.

Basco, N. and Pearson, A.E. (1967) Reactions of sulphur atoms in presence of carbon disulphide, carbonyl sulphide, and nitric oxide. Transactions of the Faraday Society 63:2684-2694.

Baughcum, S.L. and Oldenborg, R.C. (1984) Measurement of the $\mathrm{C}_{2}\left(\mathrm{a}^{3} \Pi_{\mathrm{u}}\right)$ and $\left.\mathrm{C}_{2}\left(\mathrm{X}^{1} \Sigma\right]_{g}^{+}\right)$Disappearance Rates with $\mathrm{O}_{2}$ from 298 to 1300 Kelvin, Oxford University Press, Cary, NC.

Baulch, D.L., Drysdale, D.D., and Home, D.G. (1976) Evaluated Kinetic Data for High Temperature Reactions, Butterworths, London.

Baulch, D.L., Cobos, C.J., Cox, R.A., Esser, C., Frank, P., Just, T., Kerr, J.A., Pilling, M.J., Troe, J., Walker, R.W., and Warnatz, J. (1992) Evaluated kinetic data for combustion modelling. Journal of Physical and Chemical Reference Data 21:411-734.

Baulch, D.L., Cobos, C.J., Cox, R.A., Frank, P., Hayman, G., Just, T., Kerr, J.A., Murrells, T., Pilling, M.J., Troe, J., Walker, R.W., and Warnatz, J. (1994) Evaluated kinetic data for combustion modeling. Supplement I. Journal of Physical and Chemical Reference Data 23:847-848.

Becker, K.H., Engelhardt, B., Wiesen, P., and Bayes, K.D. (1989) Rate constants for $\mathrm{CH}\left(X^{2} \Pi\right)$ reactions at low total pressures. Chem Phys Lett 154:342-348.

Benson, S.W. and Haugen, G.R. (1967) Mechanism of the hightemperature reactions between acetylene and hydrogen. The Journal of Physical Chemistry 71:4404-4411.

Berman, M.R., Fleming, J.W., Harvey, A.B., and Lin, M.C. (1982) Temperature dependence of $\mathrm{CH}$ radical reactions with $\mathrm{O}_{2}$, $\mathrm{NO}, \mathrm{CO}$ and $\mathrm{CO}_{2}$. Symposium (International) on Combustion 19:73-79.

Berndt, M.E., Allen, D.E., and Seyfried, W.E. (1996) Reduction of $\mathrm{CO}_{2}$ during serpentinization of olivine at 300 degrees $\mathrm{C}$ and 500 bar. Geology 24:351-354.
Böhland, T., Dõbe, S., Temps, F., and Wagner, H.G. (1985) Kinetics of the reactions between $\mathrm{CH}_{2}\left(\tilde{\chi}^{3} \mathrm{~B}_{1}\right)$-radicals and saturated hydrocarbons in the temperature range $296 \mathrm{~K} \leq \mathrm{T} \leq 707 \mathrm{~K}$. Berichte der Bunsengesellschaft für physikalische Chemie 89:11101116.

Braun, W., Bass, A.M., and Pilling, M. (1970) Flash photolysis of ketene and diazomethane: the production and reaction kinetics of triplet and singlet methylene. J Chem Phys 52:51315143.

Brown, R.L. and Laufer, A.H. (1981) Calculation of activation energies for hydrogen-atom abstractions by radicals containing carbon triple bonds. The Journal of Physical Chemistry 85:3826-3828.

Butler, J.E., Fleming, J.W., Goss, L.P., and Lin, M.C. (1981) Kinetics of $\mathrm{CH}$ radical reactions with selected molecules at room temperature. Chem Phys 56:355-365.

Campbell, I.M. and Gray, C.N. (1973) Rate constants for $\mathrm{O}\left({ }^{3} \mathrm{P}\right)$ recombination and association with $\mathrm{N}\left({ }^{4} \mathrm{~S}\right)$. Chem Phys Lett 18:607-609.

Canfield, D.E. (2005) The early history of atmospheric oxygen: homage to Robert A. Garrels. Annu Rev Earth Planet Sci 33: $1-36$.

Choi, Y.M. and Lin, M.C. (2005) Kinetics and mechanisms for reactions of $\mathrm{HNO}$ with $\mathrm{CH}_{3}$ and $\mathrm{C}_{6} \mathrm{H}_{5}$ studied by quantumchemical and statistical-theory calculations. International Journal of Chemical Kinetics 37:261-274.

Chung, K., Calvert, J.G., and Bottenheim, J.W. (1975) The photochemistry of sulfur dioxide excited within its first allowed

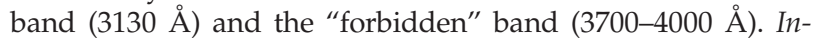
ternational Journal of Chemical Kinetics 7:161-182.

Crisp, D. (1997) Absorption of sunlight by water vapor in cloudy conditions: a partial explanation for the cloud absorption anomaly. Geophys Res Lett 24:571-574.

DeMore, W.B. and Yung, Y.L. (1982) Catalytic processes in the atmospheres of Earth and Venus. Science 217:1209-1213.

Des Marais, D.J., Harwit, M.O., Jucks, K.W., Kasting, J.F., Lin, D.N.C., Lunine, J.I., Schneider, J., Seager, S., Traub, W.A., and Woolf, N.J. (2002) Remote sensing of planetary properties and biosignatures on extrasolar terrestrial planets. Astrobiology 2:153-181.

DeWitt, H.L., Hasenkopf, C.A., Trainer, M.G., Farmer, D.K., Jimenez, J.L., McKay, C.P., Toon, O.B., and Tolbert, M.A. (2010) The formation of sulfate and elemental sulfur aerosols under varying laboratory conditions: implications for early Earth. Astrobiology 10:773-781.

Domagal-Goldman, S.D., Kasting, J.F., Johnston, D.T., and Farquhar, J. (2008) Organic haze, glaciations and multiple sulfur isotopes in the Mid-Archean era. Earth Planet Sci Lett 269:2940.

Donovan, R.J. and Husain, D. (1970) Recent advances in the chemistry of electronically excited atoms. Chem Rev 70:489-516.

Du, S., Francisco, J.S., Shepler, B.C., and Peterson, K.A. (2008) Determination of the rate constant for sulfur recombination by quasiclassical trajectory calculations. J Chem Phys 128, doi:10.1063/1.2919569.

Ekwenchi, M.M., Jodhan, A., and Strausz, O.P. (1980) Reaction of hydrogen atoms with dimethyldisulfide. International Journal of Chemical Kinetics 12:431-438.

European Space Agency. (2010) ESA-Space Science-Darwin, European Space Agency, Paris. Available online at http:// www.esa.int/science/darwin.

Fahr, A., Laufer, A., Klein, R., and Braun, W. (1991) Reaction rate of determinations of vinyl radical reactions with vinyl, methyl, and hydrogen atoms. The Journal of Physical Chemistry 95:3218-3224. 
Farquhar, J. and Wing, B.A. (2003) Multiple sulfur isotopes and the evolution of the atmosphere. Earth Planet Sci Lett 213:1-13. Farquhar, J., Peters, M., Johnston, D.T., Strauss, H., Masterson, A., Wiechert, U., and Kaufman, A.J. (2007) Isotopic evidence for Mesoarchaean anoxia and changing atmospheric sulphur chemistry. Nature 449:706-709.

Fenimore, C.P. (1969) Destruction of methane in water gas by reaction of $\mathrm{CH}_{3}$ and $\mathrm{OH}$ radicals. Symposium (International) on Combustion 12:463-467.

Gehring, Mv., Hoyermann, K., Wagner, H.G., and Wolfrum. J. (1971) Die reaktion von atomarem wasserstoff mit hydrazin. Berichte der Bunsengesellschaft für physikalische Chemie 75:12871294.

Giguere, P.T. and Huebner, W.F. (1978) A model of comet comae. I-Gas-phase chemistry in one dimension. Astrophys J 223:638-654.

Gladstone, G.R., Allen, M., and Yung, Y.L. (1996) Hydrocarbon photochemistry in the upper atmosphere of Jupiter. Icarus 119:1-52.

Gordon, S., Mulac, W., and Nangia, P. (1971) Pulse radiolysis of ammonia gas. II. Rate of disappearance of the $\mathrm{NH}_{2}\left(X^{2} \mathrm{~B}_{1}\right)$ radical. The Journal of Physical Chemistry 75:2087-2093.

Hampson, R.F. and Garvin, D. (1977) Evaluation and compilation of reaction rate data. The Journal of Physical Chemistry 81:2317-2319.

Haqq-Misra, J.D., Domagal-Goldman, S.D., Kasting, P.J., and Kasting, J.F. (2008) A revised, hazy methane greenhouse for the Archean Earth. Astrobiology 8:1127-1137.

Hills, A.J., Cicerone, R.J., Calvert, J.G., and Birks, J.W. (1987) Kinetics of the reactions of diatomic sulfur with atomic oxygen, molecular oxygen, ozone, nitrous oxide, nitric oxide, and nitrogen dioxide. The Journal of Physical Chemistry 91:1199_ 1204.

Holland, H.D. (1984) The Chemical Evolution of the Atmosphere and Oceans, Princeton University Press, Princeton, NJ.

Homann, K.H. and Wellmann, C. (1983) Kinetics and mechanism of hydrocarbon formation in the system $\mathrm{C}_{2} \mathrm{H}_{2} / \mathrm{O} / \mathrm{H}$ at temperatures up to $1300 \mathrm{~K}$. Berichte der Bunsengesellschaft für physikalische Chemie 87:609-616.

Hoyermann, K., Loftfield, N.S., Sievert, R., and Wagner, H.G. (1981) Mechanisms and rates of the reactions of $\mathrm{CH}_{3} \mathrm{O}$ and $\mathrm{CH}_{2} \mathrm{OH}$ radicals with $\mathrm{H}$ atoms. Symposium (International) on Combustion 18:831-842.

Huebner, W.F. and Giguere, P.T. (1980) A model of comet comae. II-Effects of solar photodissociative ionization. Astrophys J 238:753-762.

Intergovernmental Panel on Climate Change. (2007) Climate Change 2007: Synthesis Report. Contribution of Working Groups I, II and III to the Fourth Assessment Report of the Intergovernmental Panel on Climate Change, edited by R.K. Pachauri, A. Reisinger, and the Core Writing Team, Intergovernmental Panel on Climate Change, Geneva.

Jasper, A.W., Klippenstein, S.J., Harding, L.B., and Ruscic, B. (2007) Kinetics of the reaction of methyl radical with hydroxyl radical and methanol decomposition. J Phys Chem A 111:39323950.

Jet Propulsion Laboratory. (2010) Planet Quest: MissionsTerrestrial Planet Finder, Jet Propulsion Laboratory, Pasadena, CA. Available online at http://planetquest.jpl.nasa.gov/TPF.

Kaltenegger, L. and Sasselov, D. (2010) Detecting planetary geochemical cycles on exoplanets: atmospheric signatures and the case of $\mathrm{SO}_{2}$. Astrophys J 708:1162-1167.

Kaltenegger, L., Traub, W.A., and Jucks, K.W. (2007) Spectral evolution of an Earth-like planet. Astrophys J 658:598-616.
Kaltenegger, L., Henning, W.G., and Sasselov, D. (2010) Detecting volcanism on extrasolar planets. Astron J 140:13701380.

Kasting, J.F. (1982) Stability of ammonia in the primitive terrestrial atmosphere. J Geophys Res 87:3091-3098.

Kasting, J.F. (1990) Bolide impacts and the oxidation state of carbon in the Earth's early atmosphere. Orig Life Evol Biosph 20:199-231

Kasting, J.F. (2005) Methane and climate during the Precambrian era. Precambrian Res 137:119-129.

Kasting, J.F. and Ackerman, T.P. (1985) High atmospheric $\mathrm{NO}_{X}$ levels and multiple photochemical steady-states. J Atmos Chem 3:321-340.

Kasting, J.F. and Catling, D. (2003) Evolution of a habitable planet. Annu Rev Astron Astrophys 41:429-463.

Kasting, J.F., Liu, S.C., and Donahue, T.M. (1979) Oxygen levels in the prebiological atmosphere. J Geophys Res 84:3097-3107.

Kasting, J.F., Zahnle, K.J., and Walker, J.C.G. (1983) Photochemistry of methane in the Earth's early atmosphere. Precambrian Res 20:121-148.

Kasting, J.F., Pavlov, A.A., and Siefert, J.L. (2001) A coupled ecosystem-climate model for predicting the methane concentration in the archean atmosphere. Orig Life Evol Biosph 31: 271-285.

Kerr, J.A. and Trotman-Dickenson, A.F. (1957) The reactions of methyl radicals with thiols. The Journal of the Chemical Society 79:3322.

Kettle, A.J., Rhee, T.S., von Hobe, M., Poulton, A., Aiken, J., and Andreae, M.O. (2001) Assessing the flux of different volatile sulfur gases from the ocean to the atmosphere. J Geophys Res 106:12193-12209.

Kharecha, P., Kasting, J.F., and Siefert, J.L. (2005) A coupled atmosphere-ecosystem model of the early Archean Earth. Geobiology 3:53-76.

Krasnoperov, L.N., Chesnokov, E.N., Stark, H., and Ravishankara, A.R. (2004) Unimolecular dissociation of formyl radical, $\mathrm{HCO} \rightarrow \mathrm{H}+\mathrm{CO}$, studied over 1-100 bar pressure range. J Phys Chem A 108:11526-11536.

Kuhn, W.R. and Atreya, S.K. (1979) Ammonia photolysis and the greenhouse effect in the primordial atmosphere of the earth. Icarus 37:207-213.

Kurbanov, M.A. and Mamedov, K.H.F. (1995) The Role of the Reaction of $\mathrm{CO}+\mathrm{SH} \rightarrow \mathrm{COS}+\mathrm{H}$ in Hydrogen Formation in the Course of Interaction between $\mathrm{CO}$ and $\mathrm{H}_{2} \mathrm{~S}$, Maik Nauka/Interperiodica, Moscow.

Lam, W.W., Yokota, T., Safarik, I., and Strausz, O.P. (1989) Photolysis of and reactions of hydrogen atoms with ethanethiol. J Photochem Photobiol A Chem 47:47-63.

Lander, D.R., Unfried, K.G., Glass, G.P., and Curl, R.F. (1990) Rate Constant Measurements of $\mathrm{C}_{2} \mathrm{H}$ with $\mathrm{CH}_{4}, \mathrm{C}_{2} \mathrm{H}_{6}$, $\mathrm{C}_{2} \mathrm{H}_{4}, \mathrm{D}_{2}$, and $\mathrm{CO}$, American Chemical Society, Washington DC.

Laufer, A. (1981) Kinetics of gas phase reactions of methylene. Research on Chemical Intermediates 4:225-257.

Laufer, A.H., Gardner, E.P., Kwok, T.L., and Yung, Y.L. (1983) Computations and estimates of rate coefficients for hydrocarbon reactions of interest to the atmospheres of outer Solar System. Icarus 56:560-567.

Lawson, P.R., Lay, O.P., Johnston, K.J., and Beichman, C.A. (2007) Terrestrial Planet Finder Interferometer Science Working Group Report, Jet Propulsion Laboratory, California Institute of Technology, Pasadena, CA.

Lederberg, J. (1965) Signs of life: criterion-system of exobiology. Nature 207:9-13. 
Lee, J.H., Stief, L.J., and Timmons, R.B. (1977) Absolute rate parameters for the reaction of atomic hydrogen with carbonyl sulfide and ethylene episulfide. J Chem Phys 67:1705-1709.

Lee, L.C. (1980) $\mathrm{CN}\left(\mathrm{A}^{2} \Pi_{\mathrm{i}} \rightarrow X^{2} \Sigma^{+}\right)$and $\mathrm{CN}\left(\mathrm{B}^{2} \Sigma^{+} \rightarrow X^{2} \Sigma^{+}\right)$yields from HCN photodissociation. J Chem Phys 72:6414-6421.

Lightfoot, P.D. and Pilling, M.J. (1987) Temperature and pressure dependence of the rate constant for the addition of hydrogen atoms to ethylene. The Journal of Physical Chemistry 91:3373-3379.

Liu, Y., Wang, W.L., Wang, W.N., Luo, Q., and Li, Q.S. (2006) Density functional theory study on the biradical reaction between $\mathrm{CH}_{3} \mathrm{~S}$ and HCS. Acta Chimica Sinica 17:1785-1792.

Lloyd, A.C. (1974) Evaluated and estimated kinetic data for phase reactions of the hydroperoxyl radical. International Journal of Chemical Kinetics 6:169-228.

Lovelock, J.E. (1965) A physical basis for life detection experiments. Nature 207:568-570.

Martinez, R.I. and Herron, J.T. (1983) Methyl thiirane: kinetic gas-phase titration of sulfur atoms in $\mathrm{S}_{\mathrm{X}} \mathrm{O}_{\mathrm{Y}}$ systems. International Journal of Chemical Kinetics 15:1127-1132.

Matsumi, Y., Tonokura, K., Inagaki, Y., and Kawasaki, M. (1993) Isotopic branching ratios and translational energy release of hydrogen and deuterium atoms in reaction of oxygen $\left({ }^{1} \mathrm{D}\right)$ atoms with alkanes and alkyl chlorides. The Journal of Physical Chemistry 97:6816-6821.

McEwan, M.J. and Phillips, L.F. (1975) Chemistry of the Atmosphere, Wiley/Halsted Press, New York.

Meadows, V.S. and Crisp, D. (1996) Ground-based near-infrared observations of the Venus nightside: the thermal structure and water abundance near the surface. J Geophys Res 101:45954622.

Messing, I., Filseth, S.V., Sadowski, C.M., and Carrington, T. (1981) Absolute rate constants for the reactions of $\mathrm{CH}$ with $\mathrm{O}$ and N atoms. J Chem Phys 74:3874-3881.

Michael, J.V., Nava, D.F., Payne, W.A., and Stief, L.J. (1979) Absolute rate constants for the reaction of atomic hydrogen with ketene from 298 to $500 \mathrm{~K}$. J Chem Phys 70:5222-5227.

Miller, J.A., Mitchell, R.E., Smooke, M.D., and Kee, R.J. (1982) Toward a comprehensive chemical kinetic mechanism for the oxidation of acetylene: comparison of model predictions with results from flame and shock tube experiments. Symposium (International) on Combustion 19:181-196.

Moran, J.J., House, C.H., Vrentas, J.M., and Freeman, K. (2008) Methyl sulfide production by a novel carbon monoxide metabolism in Methanosarcina acetivorans. Appl Environ Microbiol 74:540-542.

Niki, H., Maker, P.D., Savage, C.M., and Breitenbach, L.P. (1978) Relative rate constants for the reaction of hydroxyl radical with aldehydes. The Journal of Physical Chemistry 82:132-134.

Okabe, H. (1983) Photochemistry of acetylene at $1849 \AA$ A. J Chem Phys 78:1312-1317.

Pavlov, A.A., Brown, L.L., and Kasting, J.F. (2001) UV shielding of $\mathrm{NH}_{3}$ and $\mathrm{O}_{2}$ by organic hazes in the Archean atmosphere. J Geophys Res 106:23267-23287.

Peng, J., Hu, X., and Marshall, P. (1999) Experimental and $a b$ initio investigations of the kinetics of the reaction of $\mathrm{H}$ atoms with $\mathrm{H}_{2}$ S. J Phys Chem A 103:5307-5311.

Perry, R.A. and Williamson, D. (1982) Pressure and temperature dependence of the $\mathrm{OH}$ radical reaction with acetylene. Chem Phys Lett 93:331-334.

Pilcher, C.B. (2003) Biosignatures of early earths. Astrobiology 3:471-486.

Pitts, W.M., Pasternack, L., and McDonald, J.R. (1982) Temperature dependence of the $\mathrm{C}_{2}\left(X^{1} \Sigma_{\mathrm{g}}^{+}\right)$reaction with $\mathrm{H}_{2}$ and
$\mathrm{CH}_{4}$ and $\mathrm{C}_{2}\left(X^{1} \Sigma_{\mathrm{g}}^{+}\right)$and $a^{3} \Pi_{u}$ equilibrated states) with $\mathrm{O}_{2}$ Chem Phys 68:417-422.

Prasad, S.S. and Huntress, W.T., Jr. (1980) A model for gas phase chemistry in interstellar clouds. I-The basic model, library of chemical reactions, and chemistry among $\mathrm{C}, \mathrm{N}$, and $\mathrm{O}$ compounds. Astrophys J Suppl Ser 43:1-35.

Raymond, S.N., Mandell, A.M., and Sigurdsson, S. (2006) Exotic Earths: forming habitable worlds with giant planet migration. Science 313:1413-1416.

Robie, D.C., Arepalli, S., Presser, N., Kitsopoulos, T., and Gordon, R.J. (1990) The intramolecular kinetic isotope effect for the reaction $\mathrm{O}\left({ }^{3} \mathrm{P}\right)+\mathrm{HD}$. J Chem Phys 92:7382-7393.

Robinson, T.D., Meadows, V.S., Crisp, D., Deming, D., A'Hearn, M.F., Charbonneau, D., Livengood, T.A., Seager, S., Barry, R.K., Hearty, T., Hewagama, T., Lisse, C.M., McFadden, L.A., and Wellnitz, D.D. (2011) Earth as an extrasolar planet: Earth model validation using EPOXI Earth observations. Astrobiology 11.

Romani, P.N., Bishop, J., Bezard, B., and Atreya, S. (1993) Methane photochemistry on Neptune-ethane and acetylene mixing ratios and haze production. Icarus 106:442-463.

Rothman, L.S., Jacquemart, D., Barbe, A., Chris Benner, D., Birk, M., Brown, L.R., Carleer, M.R., Chackerian, J.C., Chance, K., Coudert, L.H., Dana, V., Devi, V.M., Flaud, J.-M., Gamache, R.R., Goldman, A., Hartmann, J.-M., Jucks, K.W., Maki, A.G., Mandin, J.-Y., Massie, S.T., Orphal, J., Perrin, A., Rinsland, C.P., Smith, M.A.H., Tennyson, J., Tolchenov, R.N., Toth, R.A., Vander Auwera, J., Varanasi, P., and Wagner, G. (2005) The HITRAN 2004 molecular spectroscopic database. J Quant Spectrosc Radiat Transf 96:139-204.

Sagan, C., Thompson, W.R., Carlson, R., Gurnett, D., and Hord, C. (1993) A search for life on Earth from the Galileo spacecraft. Nature 365:715-721.

Sander, S.P., Friedl, R.R., Golden, D.M., Kurylo, M.J., Moortgat, G.K., Keller-Rudek, H., Wine, P.H., Ravishankara, A.R., Kolb, C.E., Molina, M.J., Finlayson-Pitts, B.J., Huie, R.E., and Orkin, V.L. (2006) Chemical kinetics and photochemical data for use in atmospheric studies : evaluation number 15, JPL Publication 06-2, Jet Propulsion Laboratory, California Institute of Technology, Pasadena, CA.

Schofield, K. (1973) Evaluated chemical kinetic rate constants for various gas phase reactions. Journal of Physical and Chemical Reference Data 2:25-84.

Schopf, J.W., editor. (1983) Earth's Earliest Biosphere: Its Origin and Evolution, Princeton University Press, Princeton.

Segura, A., Kasting, J.F., Meadows, V., Cohen, M., Scalo, J., Crisp, D., Butler, R.A.H., and Tinetti, G. (2005) Biosignatures from Earth-like planets around M dwarfs. Astrobiology 5:706-725.

Sharpe, S.W., Johnson, T.J., Sams, R.L., Chu, P.M., Rhoderick, G.C., and Johnson, P.A. (2004) Gas-phase databases for quantitative infrared spectroscopy. Appl Spectrosc 58:14521461.

Shum, L.G.S. and Benson, S.W. (1985) The pyrolysis of dimethyl sulfide, kinetics and mechanism. International Journal of Chemical Kinetics 17:749-761.

Singleton, D.L. and Cvetanovic, R.J. (1988) Evaluated chemical kinetic data for the reactions of atomic oxygen $\mathrm{O}\left({ }^{3} \mathrm{P}\right)$ with sulfur containing compounds. Journal of Physical and Chemical Reference Data 17:1377-1437.

Stachnik, R.A. and Molina, M.J. (1987) Kinetics of the reactions of mercapto radicals with nitrogen dioxide and oxygen. The Journal of Physical Chemistry 91:4603-4606.

Stephens, J.W., Hall, J.L., Solka, H., Yan, W.B., Curl, R.F., and Glass, G.P. (1987) Rate constant measurements of reactions of 
ethynyl radical with hydrogen, oxygen, acetylene and nitric oxide using color center laser kinetic spectroscopy. The Journal of Physical Chemistry 91:5740-5743.

Stief, L.J. and Payne, W.A. (1976) Absolute rate parameters for the reaction of atomic hydrogen with hydrazine. J Chem Phys 64:4892-4896.

Sun, F., DeSain, J.D., Scott, G., Hung, P.Y., Thompson, R.I., Glass, G.P., and Curl, R.F. (2001) Reactions of $\mathrm{NH}_{2}$ with $\mathrm{NO}_{2}$ and of $\mathrm{OH}$ with $\mathrm{NH}_{2} \mathrm{O}$. J Phys Chem A 105:6121-6128.

New Worlds Observer Team. (2010) New Worlds. New Worlds Observer Team, Boulder, CO. Available online at http:// newworlds.colorado.edu.

Toon, O.B., Kasting, J.F., Turco, R.P., and Liu, M.S. (1987) The sulfur cycle in the marine atmosphere. J Geophys Res 92:943963.

Trainer, M.G., Pavlov, A.A., DeWitt, H.L., Jimenez, J.L., McKay, C.P., Toon, O.B., and Tolbert, M.A. (2006) Organic haze on Titan and the early Earth. Proc Natl Acad Sci USA 103:1803518042.

Tsang, W. and Hampson, R.F. (1986) Chemical kinetic data base for combustion chemistry. Part I. Methane and related compounds. Journal of Physical and Chemical Reference Data 15:1087-1279.

Tsang, W. and Herron, J.T. (1991) Chemical kinetic data base for propellant combustion I. Reactions involving $\mathrm{NO}, \mathrm{NO}_{2}, \mathrm{HNO}$, $\mathrm{HNO}_{2}, \mathrm{HCN}$ and $\mathrm{N}_{2} \mathrm{O}$. Journal of Physical and Chemical Reference Data 20:609-663.

Turco, R.P., Whitten, R.C., and Toon, O.B. (1982) Stratospheric aerosols: observation and theory. Reviews of Geophysics and Space Physics 20:233-280.

Turnipseed, A.A., Barone, S.B., and Ravishankara, A.R. (1996) Reaction of $\mathrm{OH}$ with dimethyl sulfide. 2. Products and mechanisms. The Journal of Physical Chemistry 100:14703-14713.

Vance, S., Christensen, L.E., Webster, C.R., and Sung, K. (2011) Volatile organic sulfur compounds as biomarkers complementary to methane: infrared absorption spectroscopy of $\mathrm{CH}_{3} \mathrm{SH}$ enables in-situ measurements on Earth and Mars. Planet Space Sci 59:299-303.

Vaghjiani, G.L. and Ravishankara, A.R. (1990) Photodissociation of $\mathrm{H}_{2} \mathrm{O}_{2}$ and $\mathrm{CH}_{3} \mathrm{OOH}$ at $248 \mathrm{~nm}$ and $298 \mathrm{~K}$-quantum yields for $\mathrm{OH}, \mathrm{O}\left({ }^{3} \mathrm{P}\right)$ and $\left.\mathrm{H}^{2} \mathrm{~S}\right)$. J Chem Phys 92:996-1003.

Wagner, A.F. and Wardlaw, D.M. (1988) Study of the recombination reaction methyl + methyl $\rightarrow$ ethane. 2. Theory. The Journal of Physical Chemistry 92:2462-2471.

Walker, J.C.G. (1977) Evolution of the Atmosphere, Macmillan, New York.

Warnatz, J. (1984) Rate coefficients in the C/H/O system. In Combustion Chemistry, edited by W.C. Gadner Jr., SpringerVerlag, New York, pp 197-360.

Washida, N. (1981) Reaction of ethanol and $\mathrm{CH}_{3} \mathrm{CH}(\mathrm{OH})$ radicals with atomic and molecular oxygen. J Chem Phys 75:27152722.

Watkins, K.W. and Word, W.W. (1974) Addition of methyl radicals to carbon monoxide: chemically and thermally acti- vated decomposition of acetyl radicals. International Journal of Chemical Kinetics 6:855-873.

Wen, J.-S., Pinto, J.P., and Yung, Y.L. (1989) Photochemistry of $\mathrm{CO}$ and $\mathrm{H}_{2} \mathrm{O}$ - analysis of laboratory experiments and applications to the prebiotic Earth's atmosphere. J Geophys Res 94:14957-14970.

Westall, F. (2005) Evolution: life on the early Earth: a sedimentary view. Science 308:366-367.

Whytock, D.A., Payne, W.A., and Stief, L.J. (1976) Rate of the reaction of atomic hydrogen with propyne over an extended pressure and temperature range. J Chem Phys 65:191-195.

Wine, P.H., Chameides, W.L., and Ravishankara, A.R. (1981) Potential role of $\mathrm{CS}_{2}$ photooxidation in tropospheric sulfur chemistry. Geophys Res Lett 8:543-546.

Woiki, D. and Roth, P. (1995) Oxidation of $\mathrm{S}$ and $\mathrm{SO}$ by $\mathrm{O}_{2}$ in high-temperature pyrolysis and photolysis reaction systems. International Journal of Chemical Kinetics 27:59-71.

Yung, Y.L. and Demore, W.B. (1982) Photochemistry of the stratosphere of Venus-implications for atmospheric evolution. Icarus 51:199-247.

Yung, Y.L., Allen, M., and Pinto, J.P. (1984) Photochemistry of the atmosphere of Titan-comparison between model and observations. Astrophys J Suppl Ser 55:465-506.

Zabarnick, S., Fleming, J.W., and Lin, M.C. (1986) Kinetic study of the reaction $\mathrm{CH}\left(X^{2} \Pi\right)+\mathrm{H}_{2} \leftrightarrow \mathrm{CH}_{2}\left(X^{3} B_{1}\right)+\mathrm{H}$ in the temperature range 372 to $675 \mathrm{~K}$. J Chem Phys 85:4373-4376.

Zabarnick, S., Fleming, J.W., and Lin, M.C. (1989) Kinetics of CH radical reactions with $\mathrm{N}_{2} \mathrm{O}, \mathrm{SO}_{2}, \mathrm{OCS}, \mathrm{CS}_{2}$, and $\mathrm{SF}_{6}$. International Journal of Chemical Kinetics 21:765-774.

Zahnle, K.J. (1986) Photochemistry of methane and the formation of hydrocyanic acid (HCN) in the Earth's early atmosphere. J Geophys Res 91:2819-2834.

Zahnle, K.J. and Kasting, J.F. (1986) Mass fractionation during transonic escape and implications for loss of water from Mars and Venus. Icarus 68:462-480.

Zahnle, K.J., Claire, M.W., and Catling, D.C. (2006) The loss of mass-independent fractionation in sulfur due to a Palaeoproterozoic collapse of atmospheric methane. Geobiology 4:271-283.

Zhang, Q., Sun, T., Zhou, X., and Wang, W. (2005) Rate parameters and branching ratios for the multiple-channel reaction of dimethyl sulfide DMS with atomic H. Chem Phys Lett 414:316-321.

Address correspondence to: Shawn D. Domagal-Goldman NASA Headquarters 300 E St. SW Washington, DC 20056

E-mail: shawn.goldman@gmail.com

Submitted 15 June 2010 Accepted 19 March 2011 
